Florida International University FIU Digital Commons

\title{
The Development of Rubrics to Measure Undergraduate Students' Global Awareness and Global Perspective: A Validity Study
}

Stephanie Paul Doscher

Florida International University, sdoscher@fiu.edu

DOI: $10.25148 /$ etd.FI12050125

Follow this and additional works at: https://digitalcommons.fiu.edu/etd

\section{Recommended Citation}

Doscher, Stephanie Paul, "The Development of Rubrics to Measure Undergraduate Students' Global Awareness and Global Perspective: A Validity Study" (2012). FIU Electronic Theses and Dissertations. 588.

https://digitalcommons.fiu.edu/etd/588 


\section{FLORIDA INTERNATIONAL UNIVERSITY \\ Miami, Florida}

THE DEVELOPMENT OF RUBRICS TO MEASURE UNDERGRADUATE

STUDENTS' GLOBAL AWARENESS AND GLOBAL PERSPECTIVE: A VALIDITY

STUDY

A dissertation submitted in partial fulfillment of

the requirements for the degree of

DOCTOR OF EDUCATION

in

EDUCATIONAL ADMINISTRATION AND SUPERVISION

by

Stephanie Paul Doscher

2012 
To: Dean Delia C. Garcia

College of Education

This dissertation, written by Stephanie Paul Doscher, and entitled The Development of Rubrics to Measure Undergraduate Students' Global Awareness and Global Perspective: A Validity Study, having been approved in respect to style and intellectual content, is referred to you for judgment.

We have read this dissertation and recommend that it be approved.

$\begin{array}{r}\text { Peter Cistone } \\ \hline \text { Adriana McEachern } \\ \hline \text { Joan Wynne } \\ \hline \text { Hilary Landorf, Major Professor }\end{array}$

Date of Defense: March 28, 2012

The dissertation of Stephanie Paul Doscher is approved.

Dean Delia C. Garcia
College of Education

Dean Lakshmi N. Reddi

University Graduate School

Florida International University, 2012 
(C) Copyright 2012 by Stephanie Paul Doscher

All rights reserved. 


\section{DEDICATION}

For Elizabeth, Thor, and Colin, my past and my future, who always remind me to live in the present. 


\section{ACKNOWLEDGMENTS}

This is neither an exhaustive nor definitive list of acknowledgments. Five years ago the moments described below, for which I'm deeply grateful, may not have seemed significant nor have even yet occurred. Five years from now they may have faded from my poor memory. But right now, as I write these words, these are the moments that feel like gifts and these are the people who gave them to me. This is the approximate sequence of events that nudged me along towards this moment, right now:

Driving on Collins Avenue in Miami Beach with Susannah Troner. This was the first time I told anyone I wanted to pursue a doctoral degree. I remember forming the idea in my head as I was speaking the words to you, but you reacted as if you'd always known it was the true and right thing for me to do. After that day, I thought so, too.

Walking out of class one night from ZEB 325 with Anthony Normore. You looked down at me and told me I had potential as a scholar. You offered to serve as my mentor. I had no idea I needed a mentor, much less what one could do for me. You taught me what it meant to be a scholar and you made me want to be one.

Finding Donald Cramp in the halls of the Graham Center. You listened to what had happened, you took my hand, and you made sure I got home. I needed a hero that night, and you calmly rescued me. This is the moment that is hardest to remember and impossible to forget.

Eating breakfast with Anne Wolf and Ken Gold at a diner in New York City. I was done. I wanted to give up and you both understood why. But you told me to continue, to not even take a break. When we parted I hadn't decided what to do. If you'd recommended I quit, that's the decision I would have made. I couldn't let you down. 
Sitting in my red leather chair, being interviewed by Nella Roberts. You were studying how spirituality affected doctoral students' decisions to persist following transformational experiences. Before the interview I knew I'd changed; I felt defeated, like I'd lost my spirit. Somehow, afterward, I felt special. I'd been given a once-in-alifetime opportunity to figure out what really mattered to me, what would give my life purpose. Just like that, I had hope again.

Standing on the porch of my mother's house in Friday Harbor, talking on the phone with Hilary Landorf. I hung up, walked back into the living room, and told Mom and Ken and Susan Christison-Williamson, "I think my life just changed." It had, for the better, and in just about every way imaginable. How many ways are you important to me? Teacher, mentor, boss, role model, co-author, advisor, but most importantly, friend. Dear, deep, life-long friend. You've always got my back. I will always, always have yours.

Seeing "Isadore Newman" on caller ID, nearly every day, for over a year. What do people do when they don't have an Isadore Newman? That we met at FIU is a small world moment, but you opened my mind to a huge world of knowledge and skills I never thought myself capable of mastering. Your teachings and your confidence enrich my life beyond measure, personally and professionally. I owe you a lifetime supply of oatmeal raisin cookies.

Talking by Tikkun, in the sun, with Henry Richardson. I thought I was supposed to be the champion of your work, but you became the steady champion of mine. You just plain bugged me to get my butt in gear and get finished. Well check it out, buddy, I just did. So there. 
Trying to make things perfect for Drs. Linda Bliss, Peter Cistone, Adriana McEachern, and Joan Wynne. Tough, honest critics all, your opinions mean the world to me. As teachers, as committee members, and in some cases, as colleagues, your guidance and feedback have significantly influenced my development as a scholar and professional. I'll tear my last hair out to ensure my work meets your high standards.

Making waffles in the new kitchen with Laurie Paul, Tim Barrette, Sam PaulBarrette, and Talulah Paul-Barrette. I don't think I could have returned to Miami and worked as long and as hard as I did to finish if I hadn't had a mind and heart full of memories from the holidays in Friday Harbor. Every visit we share is special, but this last one was even more so, for more reasons than I have room to describe. I'm counting the days until summer.

Becoming "a doctor in the family" for Mom and Dad. From watching Colin while I went to class, to loaning me money, to not pestering me about when I'd finish, you have both completely and perfectly supported my work over the past eight years. There is no one I'd rather hear speak the words "Dr. Doscher" than each of you.

Everything, anything, with Colin Doscher. You probably can't even remember a time when I wasn't preoccupied with my schoolwork, can you? You've been so patient, so understanding, you've taken all of my stress and bother in stride. Well, this part of the journey is over, son. My days of being a student are in the rearview mirror. The only diplomas I see on the horizon are yours. Now go get that horizon... 


\begin{abstract}
OF THE DISSERTATION
THE DEVELOPMENT OF RUBRICS TO MEASURE UNDERGRADUATE

STUDENTS' GLOBAL AWARENESS AND GLOBAL PERSPECTIVE:
\end{abstract}

$$
\text { A VALIDITY STUDY }
$$

by

\author{
Stephanie Paul Doscher \\ Florida International University, 2012 \\ Miami, Florida \\ Professor Hilary Landorf, Major Professor
}

Higher education institutions across the United States have developed global learning initiatives to support student achievement of global awareness and global perspective, but assessment options for these outcomes are extremely limited. A review of research for a global learning initiative at a large, Hispanic-serving, urban, public, research university in South Florida found a lack of instruments designed to measure global awareness and global perspective in the context of an authentic performance assessment.

This quasi-experimental study explored the development of two rubrics for the global learning initiative and the extent to which evidence supported the rubrics' validity and reliability. One holistic rubric was developed to measure students' global awareness and the second to measure their global perspective. The study utilized a pretest/posttest nonequivalent group design. Multiple linear regression was used to ascertain the rubrics' ability to discern and compare average learning gains of undergraduate students enrolled in two global learning courses and students enrolled in two non-global learning courses. 
Parallel pretest/posttest forms of the performance task required students to respond to two open-ended questions, aligned with the learning outcomes, concerning a complex case narrative. Trained faculty raters read responses and used the rubrics to measure students' global awareness and perspective. Reliability was tested by calculating the rates of agreement among raters.

Evidence supported the finding that the global awareness and global perspective rubrics yielded scores that were highly reliable measures of students' development of these learning outcomes. Chi-square tests of frequency found significant rates of interrater agreement exceeding the study's .80 minimum requirement. Evidence also supported the finding that the rubrics yielded scores that were valid measures of students' global awareness and global perspective. Regression analyses found little evidence of main effects; however, post hoc analyses revealed a significant interaction between global awareness pretest scores and the treatment, the global learning course. Significant interaction was also found between global perspective pretest scores and the treatment. These crossover interactions supported the finding that the global awareness and global perspective rubrics could be used to detect learning differences between the treatment and control groups as well as differences within the treatment group. 


\section{TABLE OF CONTENTS}

CHAPTER

PAGE

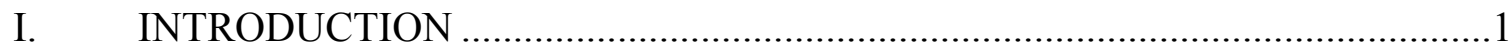

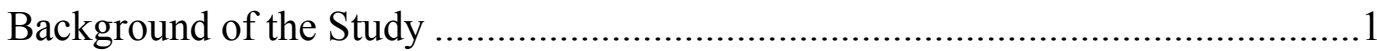

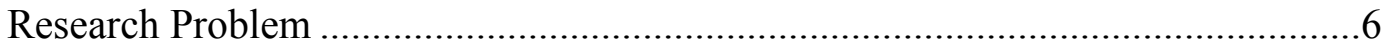

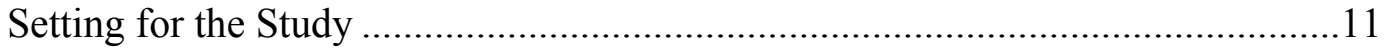

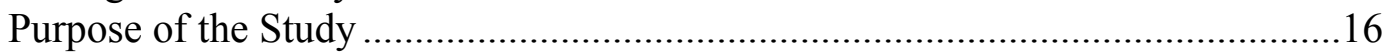

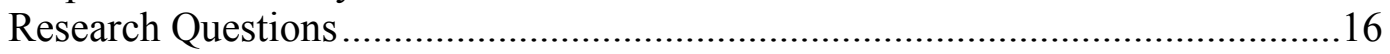

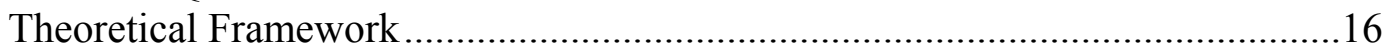

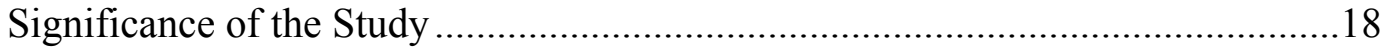

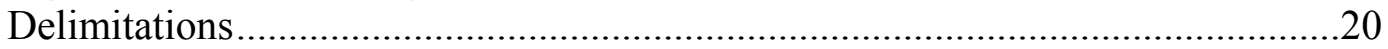

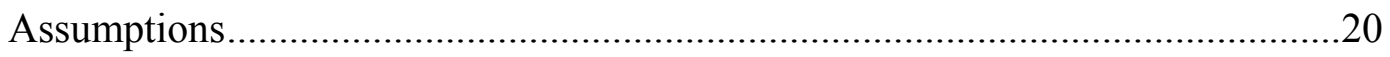

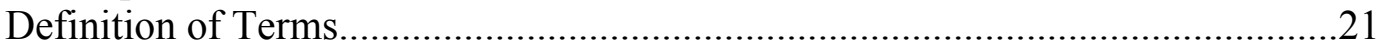

Overview of Succeeding Chapters..................................................................25

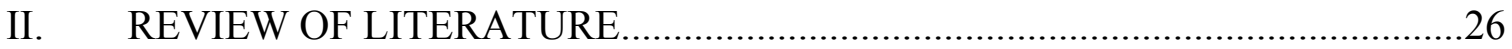

Global Awareness and Global Perspective: Defining Outcomes of

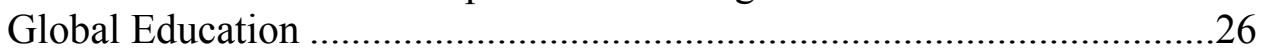

Global Learning as an Educational Process..........................................................4

Assessing Global Awareness and Global Perspective ............................................45

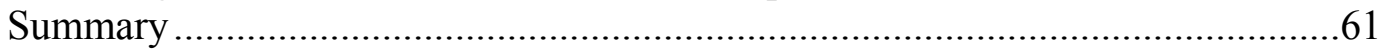

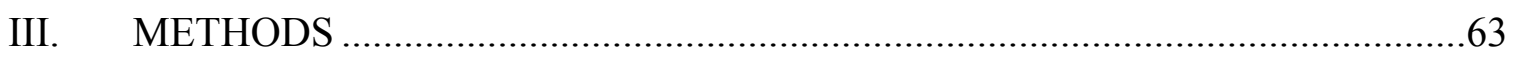

Research Questions and Hypotheses ............................................................63

Research Design...................................................................................66

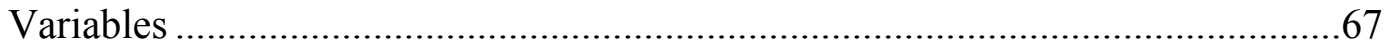

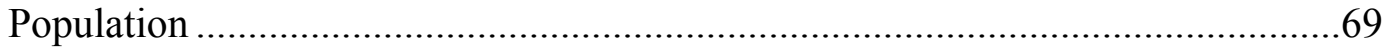

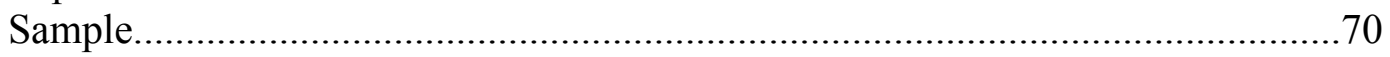

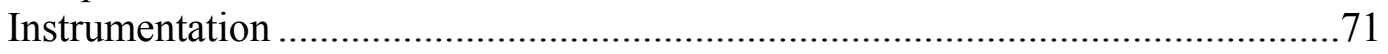

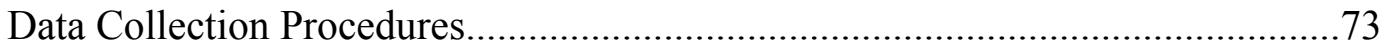

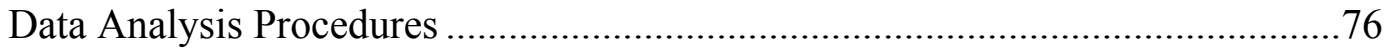

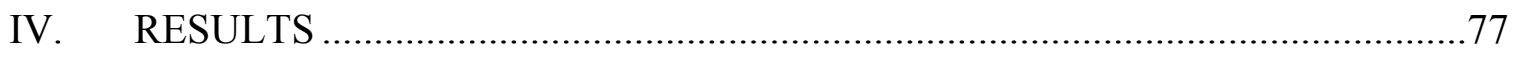

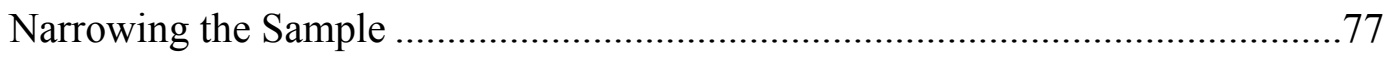

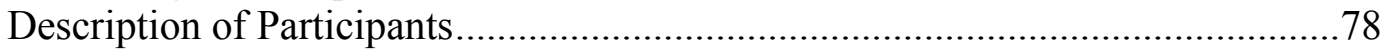

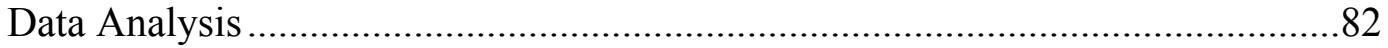

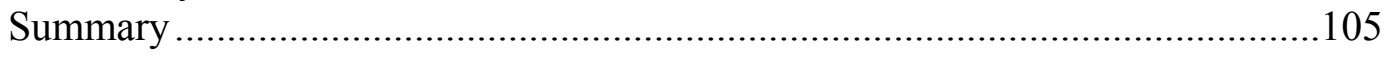




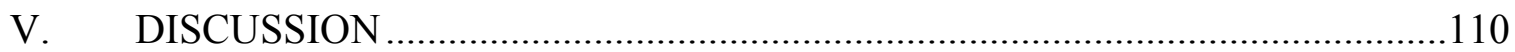

Summary of the Study ............................................................................... 110

Interpretation and Analysis of Results ..........................................................116

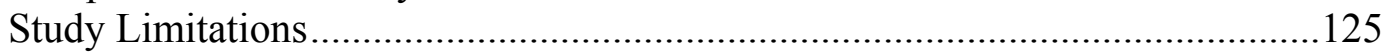

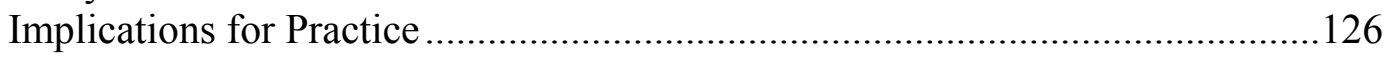

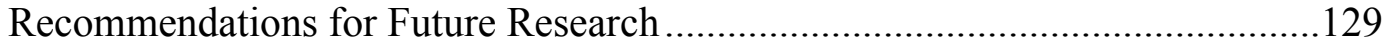

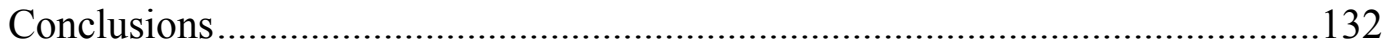

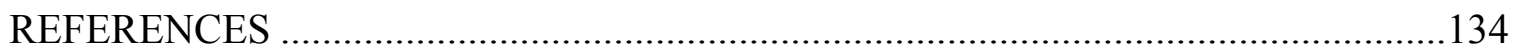

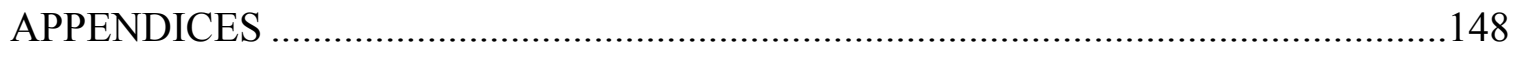

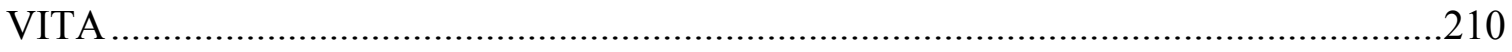




\section{LIST OF TABLES}

TABLE

PAGE

1. Descriptive Statistics of Participants, Class Status ............................................... 79

2. Descriptive Statistics of Participants, Race/Ethnicity ........................................ 80

3. Descriptive Statistics of Participants, Fluency in More Than One Language ............ 81

4. Descriptive Statistics of Participants, Time Spent Abroad ..................................... 81

5. Descriptive Statistics of Participants, Previous Global Learning Course.................. 82

6. Descriptive Statistics of Participants, Global Learning Course Grade ...................... 82

7. Hypothesis $3_{\mathrm{a}}$ : Summary of Results of Regression Analysis ............................... 85

8. Hypothesis $3_{\mathbf{a}}$ : Predictor Variables to Determine Global Awareness

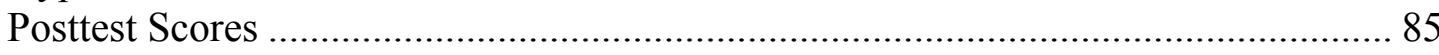

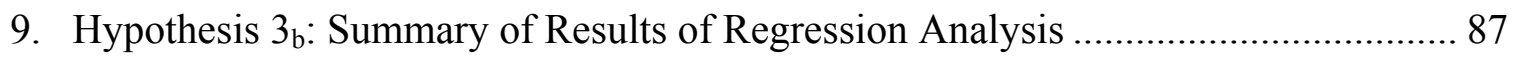

10. Hypothesis $3_{\mathrm{b}}$ : Predictor Variables to Determine Global Awareness Posttest Scores 87

11. Hypothesis 3c: Summary of Results of Regression Analysis 88

12. Hypothesis 3 : Predictor Variables to Determine Global Awareness Posttest Scores 89

13. Hypothesis $3_{\mathrm{d}}$ : Summary of Results of Regression Analysis

14. Hypothesis $3_{\mathrm{d}}$ : Predictor Variables to Determine Global Awareness Posttest Scores 90

15. Hypothesis $3_{\mathrm{e}}$ : Summary of Results of Regression Analysis

16. Hypothesis $3_{\mathrm{e}}$ : Predictor Variables to Determine Global Awareness Posttest Scores 92

17. Hypothesis 3 f: Summary of Results of Regression Analysis

18. Hypothesis 3 f: Predictor Variable to Determine Global Awareness Posttest Scores 
19. Hypothesis $4_{a}$ : Summary of Results of Regression Analysis

20. Hypothesis 4 : Predictor Variables to Determine Global Awareness Posttest Scores

21. Hypothesis $4_{b}$ : Summary of Results of Regression Analysis 97

22. Hypothesis 4 : Predictor Variables to Determine Global Awareness Posttest Scores 98

23. Hypothesis $4_{c}$ : Summary of Results of Regression Analysis 99

24. Hypothesis $4_{\mathrm{c}}$ : Predictor Variables to Determine Global Awareness Posttest Scores

25. Hypothesis $4_{d}$ : Summary of Results of Regression Analysis 101

26. Hypothesis $4_{d}$ : Predictor Variables to Determine Global Awareness Posttest Scores 101

27. Hypothesis $4 \mathrm{e}$ : Summary of Results of Regression Analysis 102

28. Hypothesis 4 : Predictor Variables to Determine Global Awareness Posttest Scores 103

29. Hypothesis $4_{\mathrm{f}}$ : Summary of Results of Regression Analysis 104

30. Hypothesis $4_{\mathrm{f}}$ : Predictor Variable to Determine Global Awareness Posttest Scores 104 


\section{LIST OF ACRONYMS}

AAC\&U American Association of Colleges and Universities

AERA American Educational Research Association

ANCOVA Analysis of Covariance

ANOVA Analysis of Variance

APA American Psychological Association

ATI Aptitude-Treatment Interaction

CMI Case Method of Instruction

CFT Cognitive Flexibility Theory

DMIS Developmental Model of Intercultural Sensitivity

DV Dependent Variable

GPI Global Perspective Inventory

IDI Intercultural Development Inventory

ISD Ill-structured Domain

IV Independent Variable

NCME National Council on Measurement in Education

SLO Student Learning Outcome

UNDP United Nations Development Programme

UNESCO United Nations Educational, Scientific and Cultural Organization

UNICEF United Nations International Children's Emergency Fund 


\section{CHAPTER I}

\section{INTRODUCTION}

This research study examined the development of rubrics to measure undergraduate students' global awareness and global perspective. More specifically, it employed an approach for estimating the validity and reliability of scores yielded from these rubrics. Chapter 1 of this dissertation provides the background of the study, the research problem and purpose, the setting, research questions, theoretical framework, and the study's significance, delimitations, and assumptions. The chapter concludes with definitions of terms and an overview of succeeding chapters.

\section{Background of the Study}

Today's young adults are citizens in a diverse and interconnected world. The issues and problems they face-whether national, international, or global in scope-are complex, ill-structured, and shaped by shifting dynamics. In order to critically respond to these challenges, undergraduates must understand how local, global, international, and intercultural issues, trends, and systems are interrelated and be able to analyze problems from multiple perspectives (Adams \& Carfagna, 2006; American Council on International Intercultural Education Conference, 1996; Hunter, White, \& Godbey, 2006). Knowledge of interrelationships among issues, trends, and systems across the globe has been called global awareness (Lemke, 2002). The ability to examine the world via diverse cultural, intellectual, and spiritual points of view has been called global perspective (Braskamp, Braskamp, \& Merrill, 2009). Increased global awareness and global perspective are often cited as among the intended student learning outcomes (SLOs) of a global education (Hovland, 2009; Loveland, 2010; Skelton, 2010). 
The Association for Curriculum Supervision and Development (Tye, 1990) has defined global education in terms of its learning outcomes, knowledge of interrelatedness and the ability to view the world through multiple perspectives:

Global education involves learning about those problems and issues which cut across national boundaries and about the interconnectedness of systems - cultural, ecological, economic, political, and technological. Global education also involves learning to understand and appreciate our neighbors who have different cultural backgrounds from ours; to see the world through the eyes and minds of others; and to realize that other peoples of the world need and want much the same things. (p. 5)

Global awareness and global perspective have also been identified as fundamental SLOs for global citizenship (Florida International University, 2010). Increasingly, students view themselves as citizens of not only local and national communities, but also the global community (Education Development Center, 2006; Our World Alliance, 2006). Students' sense of affiliation with interconnected civic spheres has significant implications for education in the $21^{\text {st }}$ century. To address this broader understanding of affiliation, colleges and universities across the United States have implemented a variety of global education initiatives - many involving global learning (Grudzinski-Hall, 2007). Global learning is the process by which students are prepared to fulfill their civic responsibilities in a diverse and interconnected world (Hovland, 2006). Global learning is also a term used to describe the specific curricular, pedagogical, and assessment strategies that enable students to develop SLOs associated with global education and 
global citizenship, that is global awareness and global perspective (Florida International University, 2010).

There is growing consensus that global learning should be part of the educational mission of all American colleges and universities (American Council on International Intercultural Education Conference, 1996; International Association of Universities, 2003; Hovland, 2006). Global learning is an educational process that was developed in response to the ways in which globalization has transformed everyday life. Many of these changes were driven by unprecedented acceleration in the pace, volume, and scale of information sharing during the $20^{\text {th }}$ century (Castells, 1999; Thompson, 2003). Thick information networks have opened individuals' eyes to diverse problems and perspectives and enabled them to develop an understanding of the interconnectivity of people, the institutions they create, and the environments in which they live. Globalization is often described in terms of the Information Revolution's macro-level impact on economies, markets, supply chains, human resource flows, consumption patterns, and cultural transfer (Chase-Dunn, 1999; Cole, 2003; Keohane \& Nye, 2000; Tomlinson, 1999), but globalization has also affected the way individuals view their relationship with other individuals and societies (Drucker, 1999). Rising numbers of American universities are initiating global learning initiatives in order to prepare students for success as global citizens.

The idea of global citizenship was born in classical Greece, but it has taken on new relevance in the era of $21^{\text {st }}$ century globalization (Appiah, 2006). Global citizenship is a distinctly different notion than that of national citizenship. Whereas national citizenship is defined as a set of rights and responsibilities granted by the nation-state, 
global citizenship is a disposition that guides individuals to take on responsibilities within interconnected local, global, intercultural, and international contexts (Steenburgen, 1994). Implicit in this concept is the idea that people are members of a larger community than that of the nation-state. Whereas national citizenship is granted by virtue of birthplace, parentage, or naturalization, global citizenship is an outlook developed through education. Nussbaum (2004) has asserted that these perceptions of global citizenship dictate the need for global learning, even in a time of cost-cutting and increasing preprofessional specialization:

Cultivating our humanity in a complex interlocking world involves understanding the ways in which common needs and aims are differently realized in different circumstances. This requires a great deal of knowledge that American college students rarely got in previous eras...We must become more curious and more humble about our role in the world, and we will do this only if undergraduate education is reformed in this direction. (p. 45)

Nussbaum (2004) has also argued that global citizens cannot function on the basis of factual knowledge alone. Global citizens need to be familiar with prevailing world conditions, but they must also understand how issues, trends, and systems are interrelated. Adams and Carfagna (2006) echoed this position in Coming of Age in a Globalized World: The Next Generation, wherein they argued that global citizens must understand contemporary interconnected local and global dynamics. Likening knowledge of interrelatedness to a connect-the-dot puzzle, the authors warned of the danger of focusing on the isolated dots, rather than the connections between and among them: 
As a society, we are flooded with information. It can be overwhelming, but it is critically important to find meaning...Without understanding relationships and connections, we are forced only to react to isolated events. We can never make decisions or act in a way that anticipates or takes advantage of trends or events. We must each therefore develop the ability to connect the dots. (p. 2) Global citizens also need to be able to view the world from multiple perspectives. One's perspective consists of ordinarily unexamined assumptions, evaluations, explanations, and conceptions of time, space, and causality (Hanvey, 1975). A person needs to develop a sense of his or her own perspective and recognize that it is shaped by multiple influences (e.g., culture, religion, gender, socioeconomic status, education) in order to develop an understanding of others' perspectives and discern their distinctive and common qualities. Perspectives determine the ways people create meaning from experience (Tomlinson, 1999). The ability to understand issues from multiple perspectives is critically important to problem solving in a diverse and interconnected world.

As a result of their global awareness and global perspective, global citizens perceive themselves as shaping the conditions of the world rather than merely navigating them. National citizenship carries with it rights and responsibilities, but global citizens are driven to define rights and take on responsibilities in multiple contexts. Understanding that they are members of interrelated communities and that others' wellbeing impacts their own, global citizens accept shared responsibility for solving problems (Hanvey, 1975). What's more, global citizens are willing to take action to solve these problems (Falk, 1994). In essence, global citizens view themselves as change agents. 
Their actions are grounded in their understanding of the interrelatedness of world conditions and their ability to approach issues from multiple points of view.

Global learning prepares students to manage the complexity, diversity, and change that define contemporary life for global citizens (National Leadership Council for Liberal Education \& America's Promise, 2007). In the past, the knowledge supply remained relatively constant. Knowledge and skills formed through a traditional liberal arts education were adequate over the long term (Brunold, 2005). However, a traditional liberal education, once deemed global because it provided a breadth of exposure to a variety of disciplines, no longer suffices. Institutions of higher learning across the United States are adopting global learning initiatives in order to prepare students to meet the challenges and opportunities of citizenship in the $21^{\text {st }}$ century (Grudzinski-Hall, 2007). These initiatives involve different components (e.g., general education reforms, certificate programs, foreign language requirements, study abroad programs, service learning programs), but they share a common purpose: to enable students to acquire the knowledge, skills, and attitudes necessary to succeed as citizens in an increasingly globalized world.

\section{Research Problem}

Global awareness and global perspective are widely acknowledged to be important SLOs of higher education. A variety of American institutions of higher learning have adopted these outcomes (Grudzinski-Hall, 2007). Institutions have developed different kinds of global learning initiatives to support student achievement of these outcomes, but when it comes to assessing student learning, the options are limited. 
In a review of global learning assessment instruments, the Global Understanding Survey (Barrows, Ager, Bennett, Braun, Clark, Harris, \& Klein, 1981), the Worldmindedness Scale (Sampson \& Smith, 1957), the Global-mindedness Scale (Hett, 1993), the Intercultural Development Inventory (Hammer, Bennett, \& Wiseman, 2006), and the Global Perspective Inventory (Braskamp, Braskamp, \& Merrill, 2009) were each found to have been used to assess students' global awareness and perspective. Although these instruments attempt to capture students' knowledge of the world and their openness to and appreciation of multiple perspectives, they do not assess global awareness and perspective as defined in this study. Moreover, all of these instruments address the knowledge and skills they purport to measure in isolation. The instruments assess global awareness and global perspective as attributes that are disconnected from one another and from the real-world problem solving context in which they are to be applied. These issues call into question the effectiveness, authenticity, and validity of these instruments as measures of global awareness and global perspective.

Effective assessment is based on the premise that learning is complex and integrative and that it involves not only what students know and can do, but also how they apply knowledge and skills to authentic tasks (American Association for Higher Education, 1991; Greater Expectations Project on Accreditation and Assessment, 2004). Authentic assessments present students with real-world tasks that require use of their collective capabilities, that is, their wide-ranging knowledge and skill base. Authentic assessments also require students to inductively develop responses based on evidence rather than allowing them to select answers from a pre-determined set, regardless of reason. Generally speaking, authentic assessments involve ill-structured challenges that 
allow students to rehearse problem solving skills tailored to the uncertainty of personal, civic, and professional tasks (Wiggins, 1990). Performance assessment is often used as a synonym for authentic assessment (Palm, 2008). Performance assessments require students "to actively accomplish complex and significant tasks, while bringing to bear prior knowledge, recent learning, and relevant skills to solve realistic or authentic problems" (Herman, Aschbacher, \& Winters, 1992, p. 2).

The above-mentioned assessment instruments do not require students to apply their global awareness and global perspective to authentic real-world tasks, therefore scores yielded from these measures may not be valid indicators of these SLOs. According to Wiggins (1990), validity should depend in part on the premise that the activity mirrors a real-world test of knowledge and skill. Validity is "an evaluative judgment of the degree to which empirical evidence and theoretical rationales support the adequacy and appropriateness of interpretations and actions based on test scores or other modes of assessment" (Messick, 1996, p. 1). Validity is not a characteristic of the instrument itself; rather, validity represents the degree to which evidence supports interpretations of assessment data and actions based on those interpretations (American Educational Research Association, American Psychological Association, \& National Council on Measurement in Education, 1999; Messick, 1996; Moskal \& Leydens, 2000). To estimate validity, researchers engage in an ongoing process of accruing evidence to support and/or refute the use of assessment results for making various types of decisions (Messick, 1996; Steen, 1999). In short, validation addresses "the appropriateness, meaningfulness, and usefulness of the specific inferences made from test scores" (American Educational Research Association, et al., 1999, p. 9). 
Messick (1996) argued that while the process is always incomplete, validation is a matter of "constructing a network of evidence supporting [or challenging] the intended purpose of the testing" (p. 1). He recommended that a construct-centered approach to valid performance assessment development should involve: (a) determination of the knowledge, skills, and/or other traits that should comprise assessable objectives within the construct; (b) identification of behaviors that would demonstrate mastery and testing situations that would facilitate those behaviors; and (c) establishment of constructrelevant scoring criteria or rubrics (Messick, 1989). Messick viewed validity as a unified concept, subsumed within a construct-driven framework. He proposed six interrelated aspects associated with construct validity that could be addressed as sources of validation evidence. These aspects are as follows:

\section{Content}

Comprised of evidence of "content relevance, representativeness, and technical quality" (p. 7).

\section{Substantive}

Encompasses "theoretical rationales for observed consistencies in test responses... empirical evidence that the theoretical processes are actually engaged by respondents in the assessment tasks" (p. 7).

\section{Structural}

Involves estimation of the "fidelity of scoring structure to the structure of the construct domain" (p. 7). 


\section{Generalizability}

Consists of the "extent to which score properties and interpretations generalize to and across population groups, settings, and tasks" (p. 7).

\section{External}

Incorporates "convergent and discriminant evidence from multitrait-multimethod comparisons, as well as evidence of criterion relevance and applied utility" (p. 7).

\section{Consequential}

Entails appraisal of "the value implications of score interpretation as a basis for action as well as the actual and potential consequences of test use" (p. 7).

Validity and reliability of performance assessments must be estimated on the basis of appropriate standardized criteria for a variety of possible student responses. One type of reliability is the consistency or agreement of scores across raters and testing situations (Moskal \& Leydens, 2000). Validity and reliability are interrelated concepts in that estimates of reliability affect estimates of validity. For example, a measurement instrument such as a scale may be considered highly reliable yet yield low estimates of validity. The scale might consistently register the same weight for a five-pound bag of potatoes - yet be an invalid measure of the number of potatoes in the bag. However, if an instrument is to be considered valid, it must also yield high estimates of reliability. Evidence must indicate that the test measures what it is designed to measure and that the test yields consistent results. To extend the example, if a scale registers a different weight each time a bag of potatoes is weighed, the scale would yield low estimates of validity as an instrument to measure weight. Estimates of assessment score reliability hold highstakes consequences in the estimation of validity because they influence the extent to 
which decisions made on the basis of those scores are fair and accurate. This is particularly true with large-scale assessments, where decisions based on student learning data are often significant and irreversible (Jonsson \& Svingby, 2007).

Given the proliferation of global learning initiatives across the United States and the dearth of appropriate assessments, there is a need for authentic instruments for the measurement of students' global awareness and global perspective. Moreover, there is a need for studies that allow researchers and educational decision-makers to estimate the extent to which data yielded from these instruments represent valid and reliable measurements of students' global awareness and global perspective.

\section{Setting for the Study}

Global awareness and global perspective were two SLOs for an institution-wide global learning initiative at a large, Hispanic-serving, urban, public, research university in South Florida. The purpose of this particular initiative was to provide all undergraduate students with curricular and co-curricular opportunities to develop these outcomes through global learning. All undergraduates, including transfer students, took a minimum of two global learning courses - one as part of the general education curriculum and one as part of their major program of study—and participated in co-curricular activities designed to increase their global awareness and global perspective. Global learning courses were developed to enhance students' global awareness and global perspective through components such as international and global content, active learning strategies, team teaching, integrated co-curricular activities, and interdisciplinary and problem-based curricula. 
The university had an established process for developing and approving global learning courses. Faculty and student affairs staff members who were developing new courses and activities, or who were revising existing courses and activities for global learning, participated in interdisciplinary and interdepartmental workshops, for which they received a stipend. Workshop participants learned how to develop course and activity outcomes aligned with the initiative's global awareness and global perspective SLOs, and they also learned how to implement active learning strategies, interdisciplinary and global content, and appropriate authentic assessments for global learning. Following the workshop, faculty and staff members designed global learning course syllabi and comprehensive assessment plans (see Appendix A) for submission to a faculty senate global learning curriculum committee. The committee assessed new and revised courses for adherence to global learning course approval guidelines. The committee used a checklist to assess discipline-specific global learning courses in academic programs as well as global learning courses in the general education curriculum, known as global learning foundations courses (see Appendix B).

The university's provost established an administrative office to coordinate all aspects of the global learning initiative, including the development of the global learning SLOs and the implementation of professional development workshops and pre/post student learning assessment. The provost appointed an Associate Professor of Teaching and Learning from the university's College of Education as director of the global learning office. The director appointed this researcher, a doctoral candidate in Educational Administration and Supervision, as associate director. Both the director and associate 
director possessed expertise and experience in the fields of global education and assessment.

Upon their appointment, the director and associate director led a year-long series of focus groups and discussions to determine the university's global learning SLOs. In these talks, faculty, staff, students, and other institutional stakeholders consistently cited global awareness and global perspective as among the most important learning outcomes for $21^{\text {st }}$ century undergraduates. At the end of this process, the university's faculty senate and Board of Trustees approved the following global learning SLOs:

\section{Global Awareness}

Knowledge of the interrelatedness of local, global, international, and intercultural issues, trends, and systems.

\section{Global Perspective}

The ability to conduct a multi-perspective analysis of local, global, international, and intercultural problems.

\section{Global Engagement}

The willingness to engage in local, global, international, and intercultural problem solving.

The director sought an instrument that would enable direct assessment of the knowledge (global awareness) and skill (global perspective) SLOs and indirect assessment of the attitude (global engagement) SLO. These instruments would be delivered to samples of incoming freshmen and transfer students as well as to samples of graduating seniors. This pre/post assessment model would allow stakeholders to evaluate the effect of the entire global learning program on student achievement of the SLOs. A 
review of existing instruments yielded several that could be used to assess global engagement. Following a thorough item analysis, the Global Perspective Inventory (Braskamp et al., 2009) was chosen for this purpose. The review did not reveal instruments designed to directly measure global awareness and global perspective as interrelated knowledge and skill outcomes in an authentic problem solving context. Additionally, the review did not reveal any instruments designed to measure these outcomes across the curriculum, as was needed for this initiative.

The director sought to develop an instrument in-house in order to fulfill the university's assessment needs. Based on their knowledge of global education and educational assessment, the director and associate director determined that global awareness and global perspective would be best measured by a performance assessment. Performance assessments are often evaluated using rubrics—scoring guidelines that include detailed qualitative descriptions of performance standards (Andrade, 2005; Popham, 1997). Rubrics are commonly used to measure student achievement on performance assessments (Moskal, 2000; Popp, Ryan, \& Thompson, 2009; Stellmack, Konheim-Kalkstein, Manor, Massey, \& Schmitz, 2009; Thaler, Kazemi, \& Huscher, 2009).

The director initiated the development of two rubrics—one for assessing students' global awareness and another for assessing global perspective - that could be used to evaluate a variety of performance tasks across the curriculum. For pre/post assessment of the outcomes, this researcher developed a performance task in which students were required to respond to two open-ended questions, aligned with the global learning SLOs, 
concerning complex case studies. This researcher also developed two rubrics that were used to evaluate students' written responses to the questions.

In some disciplines, case studies are used to provide students with necessary background knowledge and practice applying critical thinking skills to complex, often illstructured problems. The Case Method of Instruction (CMI) is used in professional education for many fields. CMI is used to encourage student engagement and interest in addressing real-world situations in a classroom setting. Faculty and staff use case studies to help students learn to think logically and systematically. Through discussion and analysis, students also learn to view problems in context and identify multiple theoretical and analytical perspectives pertinent to each case. Viewed through the lens of a teachercentered paradigm, $\mathrm{CMI}$ is considered a pedagogical strategy that faculty and staff use to transmit knowledge and model critical thinking processes. However, when viewed from the perspective of a learner-centered paradigm, case study analysis presents students with an opportunity to demonstrate their ability to integrate general and discipline-specific knowledge and skills in the construction of an evaluative argument (Golich, Boyer, Franko, \& Lamy, 2000; Hunter \& Freed, 2000; Wraga, 2008).

As is typical of performance assessments, written analyses of case studies allow the assessor to gain insight into the student's development of specific knowledge and skills. Performance tasks must be sufficiently complex, and take sufficient time to complete, in order to permit assessment of the knowledge and skills employed in bringing the task to completion (Messick, 1996; Wiggins, 1993). Thus described, case study analysis, such as was required of students in this study to measure their global awareness and global perspective, can be considered a type of performance assessment. 


\section{Purpose of the Study}

In a review of instruments designed to measure undergraduate students' global awareness and global perspective, none were found to use rubrics to assess these outcomes in the context of authentic performance tasks. The purpose of this study was to demonstrate the extent to which evidence supported the validity and reliability of scores yielded from rubrics developed to measure undergraduate students' global awareness and global perspective.

\section{Research Questions}

This study addressed four research questions. These research questions are as follows:

1. To what extent does evidence support the reliability of scores yielded from a rubric measuring students' global awareness?

2. To what extent does evidence support the reliability of scores yielded from a rubric measuring students' global perspective?

3. To what extent does evidence support the validity of scores yielded from a rubric measuring students' global awareness?

4. To what extent does evidence support the validity of scores yielded from a rubric measuring students' global perspective?

\section{Theoretical Framework}

Constructivism was the guiding theoretical framework of this study, specifically Cognitive Flexibility Theory (CFT), which focuses on learning and cognition in illstructured content domains (ISDs). ISDs require the individual to flexibly apply background knowledge and skills to unique cases (Spiro \& DeSchryver, 2009; Spiro, 
Vispoel, Schmitz, Samarapungavan, \& Boerger, 1987). Whereas well-structured domains such as basic math and sciences involve algorithmic, generalizable, and predictable content knowledge and processes, ISDs are characterized by a high degree of irregularity, multi-dimensionality, and interconnectedness. Examples of ISDs include the humanities, social sciences, arts, and advanced and applied sciences. According to Spiro and DeSchryver (2009), all professional domains contain aspects of ill-structuredness. Unbounded, real-world situational problem solving also presents cognitive challenges associated with ill-structuredness. CFT addresses how knowledge and skills should be organized and acquired to facilitate transfer to a range of new, unanticipated situations, (i.e., cognitive flexibility).

According to CFT, individuals must consider multiple unanticipated contextual variables in order to think critically in ISDs. CFT theorists advocate for instructional approaches that begin with multiple perspectives on subject matter rather than narrow, reductionist representations. Constructivist teaching strategies that activate interdisciplinary connections and adaptive knowledge assembly encourage intellectual independence. Pedagogical approaches aligned with CFT should also facilitate the use of context-dependent protocols. In ISDs, cases, defined as "examples, occurrences, events, occasions of use of conceptual knowledge" (Spiro, Coulson, Feltovich, \& Anderson, 1988, p. 7) are often complex and variable from one instance to the next. Exposure to and evaluation of multiple case representations is necessary to overcome overreliance on reductive cognitive schema. Given that logical reasoning based on a variety of sometimes unrelated precedent cases is required for critical thinking in ISDs, case analysis is the foundation of cognitive flexibility (Spiro \& DeSchryver, 2009; Spiro et al., 1987). 


\section{Significance of Study}

This study explored the development of two rubrics used to measure students' global awareness and global perspective. It employed a process for estimating the extent to which rubric scores represent valid and reliable measures of these SLOs. Validity and reliability are critically important to institutions when selecting and using instruments to assess learning outcomes, particularly for pre/post assessments. Colleges and universities use student learning assessment data to make a variety of decisions that directly affect curriculum, faculty development and placement, planning, and budgeting. Institutions must provide evidence to accrediting agencies, students, and other stakeholders that demonstrates the extent to which decisions made over time on the basis of assessment data are valid; in other words, decisions that are meaningful, useful, and appropriate (Messick, 1998). This must be done because these decisions have ethical, instructional, and practical implications that influence the efficacy of the educational endeavor.

The Code of Fair Testing Practices in Education was developed to establish ethical guidelines for professionals "in fulfilling their obligation to provide and use tests that are fair to all test takers regardless of age, gender, disability, race, ethnicity, national origin, religion, sexual orientation, linguistic background, or other personal characteristics" (Joint Committee on Testing Practices, 2004, p. 2). The first part of the code, "Developing and Selecting Appropriate Tests," recommends that developers provide information that users need in order to choose suitable tests. This includes an ample explanation of how test content was chosen and how the test was developed, as well as data concerning validity and reliability. Additionally, developers are urged to provide guidance concerning the interpretation and appropriate use of test results. Test 
users need all of this information to carry out their own ethical practice. After defining their needs in terms of test purpose, test sample, and content and skills to be tested, users should identify the most appropriate instrument following a detailed review of available test information. This study offers an example of ethical reporting for test developers and users.

This study also has instructional implications. Global awareness and global perspective are learning outcomes that were operationally defined in this study. However, these outcomes have not been universally defined nor adopted by all colleges and universities engaged in global learning initiatives. The university described in this study adopted these terms and definitions based on feedback gained from student, faculty, and staff focus groups, open forums, and college and departmental discussions. Assessment instruments were designed to specifically measure student achievement of the outcomes as described in these talks. The process of identifying SLOs and developing assessment instruments in-house has been found to positively impact instruction. This process has also been found to encourage faculty buy-in to assessment, resulting in increased use of student learning data to improve content and pedagogy. Moreover, it has been found to lead to increased coherence in the organization of curriculum and increased understanding of the value, as well as the limits, of assessment data (Crossley \& Wang, 2010).

Practically speaking, this study presents an example of how an institution can leverage internal resources in order to balance the need for self-improvement with the exigencies of external accountability. Over the past decade, accrediting agencies have increased pressure on institutions to assess SLOs and present evidence that assessment 
data are used to improve such areas as curriculum, instruction, resource allocation, and student services (Volkwein, 2009). The process described in this study may be useful for the development of assessment instruments for SLOs at the classroom and/or program levels. As faculty learn and implement the process, they can serve as mentors to others, empowering and capitalizing on in-house expertise (Rivas, Jones, \& Pena, 2010).

\section{Delimitations}

This study involved the development and validation of rubrics used to measure student responses to a performance assessment, namely open-ended questions concerning complex case studies. It may not be possible to generalize the findings of this study to the development of measurement criteria or the establishment of validity and reliability evidence for other types of assessment instruments. The activities described in this study related specifically to the operational definitions of two SLOs, global awareness and global perspective. It may not be possible to generalize this study's findings to other SLOs or to other operational definitions for these outcomes. In addition, this study was delimited to an ethnically and racially diverse population of students who attended a large, Hispanic-serving, urban, public, research university in South Florida. Learning gains achieved by these students may not be generalizable to other student populations in other types of institutions of higher learning.

\section{Assumptions}

The underlying premise of this study was that global learning is a substantively different educational process than that which is typically implemented in American higher education. This study was based on the idea that specific kinds of pedagogical strategies constitute global learning and that these strategies enable the development of 
global learning SLOs. Whether inside or outside of the classroom, whether studying, serving, or working at home or abroad, students need to engage with others in active, diverse, interdisciplinary, and problem-based learning environments in order to develop global awareness and a global perspective. Therefore, this study rested on the assumption that when global learning strategies as described in the study were implemented with fidelity, students would make learning gains in global awareness and global perspective.

\section{Definitions of Terms}

\section{Anchor Paper}

"Writing samples chosen to define levels of performance in the scoring rubric....anchor papers operationalize the concepts described in the language of the scoring rubric" (Popp, Ryan, \& Thompson, 2009, p. 256).

\section{Authentic Assessment}

Evaluation that involves tasks that "are either replicas of or analogous to the kinds of problems faced by adults citizens and consumers or professionals in the field" (Archbald \& Newmann, 1993, p. 206).

\section{Case Method of Instruction}

A pedagogical approach that emphasizes the teaching of problem solving and decision making skills through the analysis of real-life situations (Golich, Boyer, Franko, \& Lamy, 2000).

\section{Cognitive Flexibility Theory}

A constructivist theory based on the idea that learning in ill-structured domains is best achieved in an environment that emphasizes "the use of multiple mental and pedagogical representations; the promotion of multiple alternative systems of linkage 
among knowledge elements; the promotion of schema assembly (as opposed to the retrieval of prepackaged schemas); the centrality of 'cases of application' as a vehicle for engendering functional conceptual understanding; and the need for participatory learning, tutorial guidance, and adjunct support for aiding the management of complexity" (Spiro et al., 1988, p. 1).

\section{Constructivism}

An epistemology based on the idea that human beings generate knowledge and meaning by reflecting on experience.

\section{Global Awareness}

"Knowledge of the interrelatedness of local, global, international, and intercultural issues, trends, and systems" (Florida International University, 2010, p. 23).

\section{Global Citizenship}

The "willingness of individuals to apply their knowledge of interrelated issues, trends, and systems and multiperspective analytical skills to local, global, international, and intercultural problem solving" (Florida International University, 2010, p. 58).

\section{Global Education}

“...involves learning about those problems and issues which cut across national boundaries and about the interconnectedness of systems - cultural, ecological, economic, political, and technological. Global education also involves learning to understand and appreciate our neighbors who have different cultural backgrounds from ours; to see the world through the eyes and minds of others; and to realize that other peoples of the world need and want much the same things" (Tye, 1990, p. 5). 


\section{Global Learning}

The process by which students are prepared to fulfill their civic responsibilities in a diverse and interconnected world (Hovland, 2006).

\section{Global Perspective}

The "ability to conduct a multi-perspective analysis of local, global, international, and intercultural problems" (Florida International University, 2010, p. 23).

\section{Ill-structured Domain}

A disciplinary sphere "in which the following two properties hold: (a) each case or example of knowledge application typically involves the simultaneous interactive involvement of multiple, wide-application conceptual structures (multiple schemas, perspectives, organizational principles, and so on) ... and (b) the pattern of conceptual incidence and interaction varies substantially across cases nominally of the same type, that is, the domain involves across-case irregularity" (Spiro, Feltovich, Jacobson, \& Coulson, 1995, p. 92).

\section{Interaction}

An interdependent relationship between or among predictors (McNeil, Newman, \& Fraas, 2012).

\section{Inter-rater Agreement}

Consensus that occurs when different raters assign the same score to the same subject; in other words, raters are interchangeable (Kozolowski \& Hattrup, as cited in Fleenor, Fleenor, \& Grossnickle, 1996). 


\section{Norming}

The process of coming to consensus regarding the meaning of rubric scores and the characteristics of student responses that warrant each score.

\section{Performance Assessment}

"Product- and behavior-based measurements based on settings designed to emulate real-life contexts or conditions in which specific knowledge or skills are actually applied" (American Educational Research Association et al., 1999, p. 179).

\section{Perspective}

Ordinarily "unexamined assumptions, evaluations, explanations, and conceptions of time, space, causality, etc.” (Hanvey, 1975, p. 5)

\section{Power}

The probability that a false null hypothesis will be rejected.

\section{Quasi-experimental}

An experimental research design that does not include random assignment of subjects to comparison groups. This design allows researchers to look at relationships between variables rather than causes and effects.

\section{Reliability}

The consistency or agreement of scores across raters and testing situations is one type of reliability. Reliability in this study was operationally defined as the percentage of inter-rater agreement.

\section{Rubric}

A systematic scoring guide that quantifies qualitative criteria in order to evaluate behaviors, documents, or performances according to detailed performance standards. 


\section{Student Learning Outcome}

A measurable outcome focused on students' knowledge, skills, or attitudes after completing a degree program with the graduating student as the unit of analysis.

\section{Validity}

An evaluation of the degree to which evidence supports interpretations of assessment data and actions based on those interpretations (Messick, 1996).

\section{Well-structured Domain}

A disciplinary sphere that involves algorithmic, generalizable, and predictable content knowledge and processes (Spiro, Feltovich, Jacobson, \& Coulson, 1995, p. 92).

\section{Overview of Succeeding Chapters}

This dissertation consists of four additional chapters. Chapter 2 presents a review of related literature. The chapter includes an overview of global awareness and global perspective as defining SLOs of global education and a review of literature defining global learning as a process for developing these SLOs. Chapter 2 continues with a review of instruments used to assess global awareness and global perspective and the validity and reliability studies conducted for these instruments. Chapter 3 describes the methods that were used in this study. The chapter reviews the study's research hypotheses, research design, variables, population, sample, instrumentation, and data collection and analysis procedures. The results of the study and data analysis are presented in Chapter 4. Finally, Chapter 5 summarizes the study and research findings, provides an interpretation and analysis of the results as they relate to the relevant literature, and presents study limitations, implications for practice, and recommendations for future research. 


\section{CHAPTER II}

\section{REVIEW OF LITERATURE}

This chapter begins with an overview of global awareness and global perspective as defining SLOs of global education. It continues with a review of literature placing global learning within the context of global education and defining it as a process for developing global awareness and global perspective. It is necessary to firmly establish the theoretical consistency of these components for several reasons. Most importantly, effective student learning is based upon coherent alignment of learning outcomes, content, pedagogical strategies, and assessment (Biggs, 1999; McTighe \& Thomas, 2003). In addition, the generalizability of this study's results depends in part on the clarity of the construct upon which it is based. Coherence and clarity also have implications for the internal consistency of the study and the further research it prompts. Following this discussion, Chapter 2 continues with a review of instruments that have been used to assess global awareness and global perspective and concludes with an exploration of validity and reliability studies conducted for these instruments.

\section{Global Awareness and Global Perspective: Defining Outcomes of Global Education}

Scholars often cite the late 1960s as the period of inception for global education (Gaudelli, 2003; Tucker, 1996; Tye, 2009). Some attribute the beginning of a global view of education to the release of Apollo 8 images of Earth as a big blue marble, floating in space, devoid of arbitrary divisions such as national borders (Becker, 1969; Gaudelli, 2003). However, a close reading of the development of the concept of international education reveals the hidden origins of global education as far back as the $17^{\text {th }}$ century 
(Stomfay-Stitz, 1993; Sylvester, 2002). Through such an analysis it is also possible to see how interrelatedness and multiple perspectives - the core concepts of global awareness and global perspective - help to define global education as a unified conceptual framework.

\section{Two Approaches to the Development of International Education}

The history of the development of international education is marked by the evolution of two distinct approaches to the field: education for international understanding and education for world citizenship (Sylvester, 2002; Sylvester, 2003; Sylvester, 2005). This is evidenced by the definition for the subject heading international education as "works on education for international understanding; world citizenship, etc." (Library of Congress Authorities, 2012).

Although these two approaches have been used interchangeably to describe the nature and purposes of international education, they differ significantly in terms of their professed goals, SLOs, units of analysis, curricular and co-curricular content, and program and pedagogical planning strategies. A review of literature reveals that prior to the late 1960 's, the education for international understanding approach dominated the dialogue concerning international education. However, throughout that period and increasingly after the late 1960s, theorists around the world explored a different philosophy, one that was based on a holistic view of humanity and the environment- - the world citizenship approach to education. It is argued here that this nascent world citizenship approach ultimately gave rise to a unique framework that is now commonly referred to as global education. As a conceptual framework, global education can now be viewed as distinct from that of international education, which is still firmly rooted in the 
traditional practices associated with the education for international understanding approach.

Whereas the global education framework includes many of the ideas and practices associated with international education, the international education framework does not address the defining characteristics of global education. Apropos to this study, knowledge of the world's interrelatedness (global awareness) and the ability to view the world from multiple perspectives (global perspective) are defining SLOs of global education, yet these are seldom and cursorily addressed by proponents of international education. The education for international understanding framework provides a generalized rationale for all international activities at educational institutions. In contrast, global education is primarily concerned with educational activities and processes that lead to specific learning outcomes, such as global awareness and global perspective. This is explained in more detail in the following sections.

Education for international understanding approach. Prior to the late 1960's, the education for international understanding approach strongly influenced the development of international education. Calls for this type of education emerged with the rise of the nation-state and increased nationalistic wars in the late $19^{\text {th }}$ century (Butts, 1971). It was claimed that peaceful relations among nations could be achieved through knowledge of the "other" (Bonney, 1894; Buell, 1925; Good, 1960; Prescott, 1930). Butts (1971) described three elements of education for international understanding: (a) objective study of other societies in K-16 curricula; (b) student and faculty research and learning abroad; and (c) educational development aid. These elements fall within Arum and Van de Water's (1992) definition of international education, which was based on 
their historical review of the term: “'International education'... refers to the multiple activities, programs, and services that fall within international studies, international educational exchange and technical cooperation" (p. 202).

Proponents of the international understanding approach view the world as a composite of nations in existence and world peace as an aggregation of congenial relations between nations (Faure, Herrera, Kaddoura, Lopes, Petrovsky, Rahnema, \& Ward, 1972; Scanlon, 1960; Stoker, 1933; Wooten, 1929). Based on this worldview, educational strategies for international understanding are often carried out through bilateral and multilateral agreements between and among educational institutions, nations, NGOs, and intergovernmental agencies such as the United Nations Educational, Scientific and Cultural Organization (UNESCO), the United Nations International Children's Emergency Fund (UNICEF), and the United Nations Development Programme (UNDP). The content of this approach centers on the study of transactional issues through such disciplines as political science, economics, history, international relations, intercultural communication, and foreign languages (Butts, 1969; Kandel, 1937; Tewksbury, 1945; Torney-Purta, 1989). Fraser and Brickman (1968) referred specifically to the transactional nature of this approach in their definition of international education:

International education connotes the various kinds of relationships-intellectual, cultural, and educational — among individuals and groups from two or more nations. It is a dynamic concept in that it involves a movement across frontiers, whether by person, book or idea. International education refers to the various methods of international cooperation, understanding and exchange. (p. 1) 
This definition substantiates the assertion that the education for international understanding approach is predicated on the nation-state as the basic unit of analysis (Kandel, 1937; Torney-Purta, 1989). The strong national political interests that undergird this approach are balanced with the idea that well-being among nations is interrelatedbut only to a limited extent. Nations acknowledge interrelatedness and educate for world peace insofar as it serves their self-interests to do so (Kandel, 1955; Scanlon, 1960; Wilson \& Collings, 1963). This approach is consistent with national adherence to what Becker (1969) called the myth of self-sufficiency, which has led to international unwillingness to fully commit to the idea of interrelated global well-being. Referring to the tension between national interests and calls for education for world peace in the early to mid-20 $0^{\text {th }}$ century, Woody (as cited in Sylvester, 2002) observed that, "Educational leaders and idealists in many lands, East and West, saw the vision and were ready to work to realize it; but politicians and men of affairs were reluctant" (p. 119). Kenworthy (1951) concurred with this observation, noting that, Everywhere nationalism is a potent force, and there is still fear lest too much emphasis on education for a world society result in minimizing education for national citizenship...The one phrase which various nations seem to be willing to use is 'education for international understanding' as attested to by the adoption of this phraseology by UNESCO after long and heated debates. These words imply a less ambitious approach and one which most governments are willing to approve. (p. 200)

The concept of interrelatedness is present in a limited sense in the literature on education for international understanding, but the concept of perspective is conspicuously 
absent. There are some calls for students to acquire "universal respect for diversity" (Kenworthy, et al., as cited in Sylvester, 2003, p. 192) and "appreciation for the character, attainments, and traditions of other peoples" (Smith \& Crayton, as cited in Sylvester, 2002), but there is no discussion of the need to view the world through others' eyes nor of the role perspective plays in students' ability to understand how the world works. Theorists who adhere to the education for international understanding approach refer primarily to SLOs describing students' knowledge of world affairs, communication competencies, and attitudes consistent with peaceful international relations (Anderson, 1954; Kirkwood, 2001). Calls for students to think critically about the world's interrelatedness through diverse perspectives emerge in the literature on education for world citizenship.

Education for world citizenship approach. Whereas education for international understanding is based on a view of the world as the sum of nations in existence, education for world citizenship is concerned with the world as a totality. This is an important distinction, for as Becker (1969) has observed, "an aggregation of knowledge about the parts that make up the world is not equivalent to an understanding of the world as a whole" (p. 26).

The world citizenship approach to defining international education emerged from the idea that the planet is a single integrated entity that has been divided into individual nation-states. This view was not born with space travel. The concept of world citizenship pre-dates that of international understanding. As Fraser and Brickman (1968) noted in their documentary history of international and comparative education, "Prior to the 
nineteenth century, the terms cosmopolitanism and universalism were accepted and understood, but the idea of internationalism was virtually unknown" (pp. 18-19). Cosmopolitanism, or world citizenship, dates back to ancient Greece, but Sylvester (2002) credited John Amos Comenius (1592-1670), the Moravian bishop considered the father of universal education (Piaget, 1993; Scanlon, 1960), with pioneering the intersection between education and world citizenship. In his work, Comenius was concerned with the whole. He viewed education as the "art of teaching all things to all men" (Comenius, as cited in Piaget, 1993, p. 2). In The Way of Light, he envisioned an international university that would serve all men, regardless of race, religion, or nationality, and educate them for peace and the well-being of all humanity (Sylvester, 2002). He believed in the power of the newly invented printing press to support his pansophic ideal of a universal body of knowledge that could be applied to the solution of universally relevant problems. He even went so far as to suggest that for the good of peace and communication, all men should become bi-lingual—conversant in their home language and in an international lingua franca that would allow the propagation of ideas beyond borders. These ideas and others lead to the conclusion that Comenius saw himself as a world citizen in the contemporary meaning of the term (Sadler, 1970).

Comenius was a unique thinker in an age in which divisions on the basis of age, class, religion, and community were much more restrictive than they are today. His belief in universal education was rooted in his holistic vision of the human community. In his book The Pampaedia, Comenius (as cited in Auba, 1970) stated forthrightly his conviction that: 
It is essential that we should wish that even utterly barbarous peoples should be enlightened and liberated from the darkness due to lack of knowledge, for they are a part of the human race and the part should be like the whole; and further, the whole is not the whole if any part is lacking... whoever then does not wish to appear a half-wit or evil-minded, must wish good to all men, and not only to himself, or only to his own near ones, or only to his own nation. (p. 55)

These ideas had far reaching effect, both geographically—according to Cotton Mather, John Winthrop invited Comenius to become president of Harvard—and over the long term-Comenius' philosophy was invoked at international education forums such as the World Congress of Education of the Columbian Exposition in 1893 and the founding of UNESCO in 1945. Comenius' work established the world citizenship approach to international education. He considered education to be an ethical imperative that transcended national, gender, religious, cultural, or socio-economic borders. This approach is threaded throughout the development of the concept of international education. A prime example is found in the Russian Prince Serge Wolkonsky's (1894) welcoming comments to the World Congress of Education, wherein he exhorted that the union of the words international and educational would:

...loudly proclaim that every one of us belongs, first, to humanity, and secondly, to one or another nation; may it teach that there is more honour for any one of us in being a man than in being an American, or a Russian, or a German, or an Italian, or a Greek, or a Japanese, or whatever else it may be. (p. 38-9) The basic unit of analysis in the world citizenship approach is the individual. In this approach, human well-being is completely interrelated. This is consistent with the 
view that Earth is an integrated system. This system is influenced by interrelated global dynamics involving humans, the institutions they create, and the environment in which they live (Becker, 1969; Burn, 1980; Hicks, 2003; Kenworthy, 1951). In order to educate individuals towards this kind of global or "worldminded" view, proponents of this approach urge the study of issues, trends, and dynamics that transcend national boundaries and affect all human and environmental well-being (Gilliom, 2001; Kirkwood, 2001; Tye \& Tye, 1992). Since these complex global dynamics affect wellbeing in a variety of ways, this approach calls for interdisciplinary content and problemcentered teaching and learning strategies (Anderson, Nicklas, \& Broadford, 1994; Kerr, 1979; Lamy, 1982). Such study helps students to understand how interrelatedness works and facilitates a cognitive disposition towards world citizenship (Anderson, 1979; Davies, 2006; Murray, 1929).

By the late $20^{\text {th }}$ century, proponents of the world citizenship approach had begun to use the term global education to describe an emerging conceptual framework, yet definitional ambiguities remained. This was largely due to the fact that the world citizen approach had long been subsumed within the term international education. In an attempt to clarify, Kirkwood (2001) explained that global education had emerged from the "need for a 'civic culture' of individuals across the Earth [who] actively engage in meeting human needs" (p. 11). She claimed that global education had become a more full-bodied conceptual framework than that of international education, particularly in terms of its vision for content and its impact on individual learning. According to Kirkwood (2001), all people need access to a global education that centers on four themes: multiple perspectives, comprehension and appreciation of cultures, knowledge of global issues, 
and the world as an interrelated system. These themes could be integrated across the curriculum and into all subject areas and educational activities. This notion aligns with Anderson's (as cited in Bragaw, 2001) assertion that:

A student need not be studying things foreign or international, as we have conventionally thought of these terms, in order to be involved in global education. There are ways in which a student can study his or her own community and be as much involved in global education as when he or she is studying a community in another part of the world. (p. 2)

The weak national political interests that underlie education for world citizenship allow for a stronger focus on individual learning and individual well-being within the global system. This is based on mastery of the knowledge and skills individuals need in order to carry out their rights and responsibilities_-particularly those that arise from their sense of affiliation with interrelated human and environmental communities. Thus it is that knowledge of interrelatedness and the ability to analyze the world from multiple perspectives are defining learning outcomes for global education. Arising from the need to educate for world citizenship and giving rise to learning activities across the curriculum, global awareness and global perspective differentiate global education from international education.

\section{Global Awareness}

Global awareness has been called foundational for global citizenship (Gibson, Rimmington, \& Landwehr-Brown, 2008; Merryfield, 2008; Von Karolyi, 2008). While some have conceived of global awareness as a general familiarity with world events and information (Tucker, 1982), others describe this outcome as a kind of holistic outlook on 
diversity and interdependence (Clark, 2002; Davis \& Robinson, 2006; Howard, 2002;

Simonson, 1977). A number of global education theorists combine these two conceptsknowledge of global issues and the world as an interrelated system—which are akin to two of the themes identified by Kirkwood (2001), to define global awareness as knowledge of the interrelatedness of global dynamics.

In her work with gifted children, Roeper (2008) often observed an enhanced cognitive and emotional response to the world's complexity. She observed gifted children's propensity for grasping themselves as part of the world's interrelated system and for understanding how they are affected as individuals by global events and trends. Roeper (1992) combined these ideas in her description of global awareness as "a mind set, a way of seeing ourselves as an integral part of every aspect of the world. It is a conscious and unconscious realization that we are completely intertwined and interdependent with all things on earth" (p. 52). Roeper contended that although gifted children are born with this kind of global cognitive acuity, global awareness can be enhanced through education.

Clarke (2004) echoed Roeper's description of global awareness as a worldview but went further, defining it as the "cognitive or knowledge aspect of students' perceptions of interrelatedness" (p. 56). Gibson, et al. (2008) further refined this focus, defining global awareness as "knowledge of globalization and the resulting issues and problems that affect everyone's lives. It refers to an understanding of interconnectedness and interdependence of the world" (p. 15). These definitions specify the kind of knowledge global citizens need in order to think critically about the world. However, global awareness is seldom associated with lower order cognitive development, that is 
simple recall and comprehension of facts (Bloom, 1956). Global awareness is generally discussed as knowledge that results from critical analysis of global dynamics and enables the individual to make evaluative decisions in new, ill-defined, and unstructured situations.

Kniep (1989) did not use the term global awareness, but he did delineate specific content that should be taught in order to enable students to discern interconnectedness. He claimed that young people need to be involved in the following domains of inquiry in order to be prepared to tackle local, national, and global problems:

Human values. Study of universal human values that transcend group identity (e.g., equality, justice, liberty) and diverse cultural values that define group membership and contribute to differing worldviews (e.g., values related to aesthetics, life-style, the environment).

Global systems. Study of the workings of the four major interactive global systems: economic, political, ecological, and technological.

Global issues and problems. Study of the persistent, transnational, interconnected concerns of our age: peace and security, development, the environment, and human rights.

Global history. Study of the evolution of human values, the historical development of contemporary global systems, and the origins of current global issues and problems.

Merryfield (2008) folded this kind of focus on content into her description of global awareness as a mindset students need in order to survive in a world "increasingly characterized by economic, political, cultural, environmental, and technological 
interconnectedness" (p. 383). Merryfield contended that it is the duty of social studies teachers, in particular, to prepare students for this mindset by helping them to understand how the world affects them and how they, their community, and their nation influence others. She has presented three assumptions teachers hold concerning the characteristics of global awareness: (a) openmindedness; (b) mastery of a multi-disciplinary, global body of knowledge about how the world works; and (c) the ability to apply knowledge to authentic, relevant problems. Merryfield suggested that global awareness could be taught through specific pedagogical strategies. These include: (a) authentic engagement in projects that solve real-world global problems; (b) reflection on one's own cultural lens; and (c) exposure to primary sources from voices around the world.

Merryfield's description of the way students acquire global awareness - through examination of issues from multiple perspectives - is indicative of the close connection between this outcome and global perspective. Although global awareness and global perspective can be defined as discrete cognitive characteristics, they are each functionally necessary for the other's development. Nowhere in the literature is this more evident than in the work of Robert Hanvey, considered the father of global education.

\section{Global Perspective}

Hanvey's (1975) An Attainable Global Perspective is seminal in the literature on

global education. For Hanvey, an individual's perspective is composed of their ordinarily unexamined assumptions concerning time, space, causality, etc. A global perspective is a characteristic of a given group, and it is composed of the differentiated cognitive attributes of the individual members of that group. In order to move the group toward a 
global perspective, individuals should be educated towards development of the following capacities:

Perspective consciousness. Recognition that one has a unique perspective not necessarily shared by others.

State of the planet awareness. Alertness to the distortions associated with various means of attaining knowledge of world conditions.

Cross-cultural awareness. Understanding of the diversity of practices and beliefs in human societies.

Knowledge of global dynamics. Comprehension of key traits and mechanisms associated with change on a global scale.

Awareness of human choices. Understanding of the problems of choice associated with an expanded global perspective.

Hanvey (1975) asserted that individuals develop this suite of capacities to a greater or lesser degree throughout the course of their lives. He stated that the ultimate goal of education should be to prompt students to respond to information about the world with, at minimum, the thought that, "There may be more there than meets the eye" or "Other eyes might see it differently" (p. 2). In Hanvey's list of attributes, knowledge of global dynamics is foundational to the effort to teach people to view the world from multiple perspectives.

Case (1993) also viewed knowledge as one of the cognitive building blocks of a broader perspective. He expanded upon Hanvey's work by presenting what he perceived as two interconnected dimensions of a global perspective: the substantive (i.e., the object being viewed) and the perceptual (i.e., the lens through which the object is viewed). 
Case's substantive dimension is akin to what is defined here as global awareness. Case defined this dimension as "knowledge of various features of the world and how it works...Included in this dimension is knowledge of interconnected global systems, international events, world cultures, global geography, and so on" (p. 318). The perceptual element, however, "describes an orientation or outlook" (p. 318). Case summarized his view of a global perspective as the "capacity to see the 'whole picture' whether focusing on a local or an international matter" (p. 318). He went on to explain that this picture is constructed by making sense of the world through multiple perspectives and that one must possess certain characteristics in order to see the whole picture. These characteristics are:

Openmindedness. “... willingness to base our beliefs on the impartial consideration of available evidence" (Hare, as cited in Case, 1993, p. 321).

Anticipation of complexity. “...skepticism of explanations that fail to consider with sufficient imagination the range of interacting global factors and the breadth of plausible consequences" (Case, 1993, p. 322).

Resistance to stereotypes. “...skepticism about the adequacy of accounts of people, cultures, or nations that either are limited to a narrow range of characteristics (i.e., important features of the group are ignored) or depict little or no diversity within them (i.e., group heterogeneity is ignored)" (p. 322).

Inclination towards empathy. “...willingness and capacity to place ourselves in the role or predicament of others or at least to imagine issues from other individuals' or groups' perspectives" (p. 323). 
Non-chauvinism. “...the inclination neither to prejudice our judgments of others because we are not affiliated with them, nor to discount unfairly the interests of others even if, on occasion, they are incompatible with our own interests" (p. 323).

Case (1993) was careful to point out that although it is possible to define separate dimensions and components of a global perspective, these elements are so intertwined as to make it impossible to teach them individually. Rather, his effort in deconstructing the concept of global perspective was meant to lend credence to the idea that a global perspective is a complex notion and is a skill, as Hanvey asserted, that individuals possess in varying degrees for different reasons. In addition, Case claimed that as an SLO, global perspective is not unique to global education. All quality education is intended to move students away from "naïve, often mistaken views of the world" (p. 324) and towards more nuanced understandings based on their ability to critically analyze content from multiple perspectives.

While not using the term global perspective, Wicklund (1999) also discussed multiple perspectives as a form of perception in which:

...people can recognize that an event may be viewed, defined or perceived in more than one manner, through several social focal points. This is in contrast to the perception of an event as univocal, as having only one meaning, anchored in the presumed objective, physical nature of that event. (p. 2)

Pike and Selby (2006) also did not use the term global perspective, but the issues dimension of their four-dimension theory of global education did address multiperspective thinking. This dimension was primarily composed of: (a) knowledge of how perspectives are formed; (b) the ability to analyze, organize, and evaluate new 
information; and (c) critical consideration of other points of view. Also included in the issues dimension were knowledge of interconnections among issues, trends, and events and research and inquiry skills. The Selby and Pike (2000) model of global education was rooted in critical thinking and was based on the interrelationship between knowledge of interconnectedness and multiperspective analysis. To educate for this knowledge and skill base, the authors advocated for an active, transformative approach to learning that "entails a dynamic interaction between teachers, learners and multiple sources of information. Thus, the functions of 'teaching' and 'learning' are frequently interchangeable" (p. 140). Selby and Pike's learner-centered approach to global education pointed the way toward the formulation of global learning as an educational process designed to enable learners to construct meaning for active participation in a global society.

\section{Global Learning as an Educational Process}

Since 2001, the American Association of Colleges and Schools (AAC\&U) has led the national conversation on global learning through its Shared Futures: Global Learning and Social Responsibility initiative. Colleges and universities widely adhere to AAC\&U's conception of global learning as the process by which students are prepared to fulfill their civic responsibilities in a diverse and interconnected world (Hovland, 2006). Although global awareness and a global perspective are commonly cited as the knowledge and skills that should result from global learning (Hovland, 2009; Loveland, 2010; Skelton, 2010), there is far less agreement upon the nature of the process that should be implemented in order to encourage student achievement of these outcomes. 
When the concept of global learning was first introduced, educators discussed it in terms of the strategies associated with a traditional international education conceptual framework: study abroad, language instruction, and area studies programs (Cornwell \& Stoddard, 1999; Hovland, 2009). Increasingly, global learning theorists are attempting to discern the cognitive behaviors inherent in global learning outcomes, identify effective teaching and learning strategies that involve these behaviors, and apply these strategies across the curriculum and co-curriculum (Gibson, et al., 2008; Loveland, 2010). This work is more aligned with the global education conceptual framework that views education as a process. The strategies identified by these researchers have been grouped under the term global learning. In the process of global learning, SLOs drive the way educators design curriculum, pedagogy, and assessments, as well as the ways institutions integrate curriculum and co-curriculum. According to AAC\&U, global learning features "a close alignment between professed goals and actions taken to achieve those goals" (Musil, 2006, p. 4).

Global learning theorists consider global awareness and global perspective to be interrelated SLOs, therefore experiences that strengthen one outcome also serve to support the development of the other. Gibson, et al. (2008) asserted that the cognitive behaviors underlying these outcomes are those associated with critical thinking. These researchers identified specific global learning conditions that enable students to gain an understanding of the world's interconnectedness through activities that require them to view the world from multiple perspectives. These conditions are:

Cultural contrast. Also known as culture shock, cultural contrast prompts students to examine the extent of difference between values and beliefs on issues. The 
greater the frequency and intensity of contrast, the more memorable and meaningful the global learning experience.

Modern communication technologies. Methods such as email, videoconferencing, and web-based threaded discussions can enable students to experience cultural contrast without leaving their home environment.

Substantive and authentic goals. Effective global learning experiences engage students in real-world endeavors that are too complex and extensive for an individual to complete independently. Ideally, these projects should have intercultural ramifications and require a wide range of expertise to plan, design, and implement.

Teamwork. Global learning teams should be composed of members from different countries. The challenges associated with intercultural communication, particularly when different languages and communication technologies are involved, sensitize students to the need to improve communication for the sake of collaboration.

Gibson, et al. (2008) claimed that global learning is actually enhanced when learners remain situated in their home environment, as enculturation may inhibit students' ability to discern their own perspective. Merryfield (2008) also called for students to reflect on their own and others' perceptual lenses within the classroom setting. She recommended the exploration of themes through source materials from various cultural, political, and historical perspectives. Echoing the Gibson, et al. (2008) claim that global learning is enhanced through teamwork, Merryfield asserted that global awareness becomes significant when students collaborate to address authentic problems for the common good. 
Interdisciplinary investigation of problems has also become a hallmark of global learning. Multiple institutions have established interdisciplinary centers and programs in order to teach for global awareness and perspective (American Association of Colleges and Universities, 2005). Hovland (2009) asserted that interdisciplinary problems are particularly important for global learning because they demand that students bring multiple disciplinary frameworks to bear on a particular issue. In so doing, undergraduates learn to compare different disciplinary tools and perspectives. This is consistent with AAC\&U's recommendation in College Learning for the New Global Century (National Leadership Council for Liberal Education \& America's Promise, 2007) that students engage with big, complex questions that are both contemporary and enduring.

In "Learning for Democracy: From World Studies to Global Citizenship," Holden (2000) explored how global learning requires active, rather than passive learning strategies. Bonwell and Eison (1991) defined active learning strategies as those that demand students go beyond simply listening, requiring that they "read, write, discuss, or be engaged in solving problems. Most important, to be actively involved, students must engage in such higher order thinking tasks as analysis, synthesis, and evaluation” (p. iii). Typical active learning strategies used in higher education include debates, peer teaching, case analysis, service learning, role playing and simulations, computer-based instruction, and cooperative and team-based learning. Lantis, Kille, and Krain (2010) asserted that active learning strategies increase comprehension, help students make connections between theory and practice, and increase knowledge retention. In particular, case study 
and problem-based learning encourage these outcomes through critical thinking and multiperspective analysis (Lamy, 2007).

Given that the ultimate goal of global learning as an educational process is for students to acquire the knowledge and skills necessary to function successfully as global citizens, responsibility for global learning lies not only with faculty but also with student affairs professionals. With a common focus on global learning SLOs, academic and student affairs professionals can work together to provide a wide variety of venues for students to rehearse and reinforce their global awareness and global perspective (Bowen, 2005; Braskamp, 2010; Hovland, 2009; Temple-Thurston, 2005). This integrated curricular and co-curricular global learning approach aligns with the "Principles of Excellence" outlined in AAC\&U's report, College Learning for the New Global Century (National Leadership Council for Liberal Education \& America's Promise, 2007), which recommended making SLOs a framework for the entire educational experience. It also adheres to the Learning Reconsidered (Keeling, 2004) paradigm under which many university student affairs divisions operate. Learning Reconsidered was an argument for the integrated use of all of higher education's resources in the education and preparation of the whole student. It defined learning as a comprehensive, holistic, transformative activity that integrates academic learning and personal development, processes that have long been considered separate and even independent of each other.

\section{Assessing Global Awareness and Global Perspective}

In College Learning for the New Global Century, AAC\&U (National Leadership Council for Liberal Education \& America's Promise, 2007) recommended that essential SLOs form the basis of both educational intentionality and accountability across the 
institution. Additionally, the report's authors asserted that, "The right standard for both assessment and accountability at the college level is students' demonstrated ability to apply their learning to complex, unscripted problems in the context of their advanced studies" (p. 40). In its recommendation, AAC\&U suggested that essential SLOs be assessed to this standard at milestones within students' major program of study, and that such assessments be incorporated into graduation requirements. This suggestion called forth two exigencies: one, assessments for common SLOs must address differentiated content across fields of study, and two, these assessments must be held to common criteria in order to comparatively analyze and strengthen student achievement as part of the cycle of continuous improvement.

Assessments of global awareness and global perspective must reliably accommodate both of these needs—-differentiated content and common criteria—in order to be useful for global learning initiatives in higher education. Instruments must be tightly aligned with the constructs upon which these SLOs are based in order for student scores to be valid and meaningful. Interpretation of test results must enable faculty, staff, and other institutional stakeholders to make practical and effective improvements to content and teaching strategies in order to improve global learning. Institutions have made use of a variety of instruments to assess global learning, and these instruments need to be explored in terms of their validity and reliability, as well as their meaningfulness and utility, as measurements of global awareness and global perspective.

Following is a review of instruments that have been used to assess college and university students' global awareness and global perspective. These assessments were chosen for review because they were found most closely aligned with this study's 
definitions of global awareness and global perspective. Instruments that purported to assess these outcomes, even if they included the terms global awareness or global perspective in their titles, yet were not based on similar constructs were not reviewed herein. Included in the review of each instrument is a description of the conceptual framework upon which it is based; an explanation of how the test was developed; a review of the types of studies used to establish its validity and reliability; and, a determination of its construct validity as an assessment of global awareness or global perspective as defined in this study.

\section{Global Understanding Survey}

The Global Understanding Survey (Barrows, et al., 1981) was a landmark instrument developed to assess "what college students actually know and perceive about global relationships and to measure their comprehension of current global complexities" (Barrows, Clark, \& Klein, 1980, p. 10). The survey was a response to a request made by the Education and the World View project of the Council on Learning for an assessment of global understanding. The purpose of the initiative was to identify ways that higher education professionals could remedy what was then perceived as a deficit in college students' global understanding. The authors of the instrument acknowledged from the outset that a significant goal of their enterprise was to determine the meaning of global understanding, an oft-used but previously undefined term.

Barrows, et al. (1981) based their work on the premise that global understanding is a complex construct consisting of both cognitive and affective domains. In order to develop items for the instrument, the authors operationally defined global understanding as a function of four components: (a) knowledge; (b) attitudes and perceptions; (c) 
general background correlates; and, (d) language proficiency. Empirical data gathered from student responses on the instrument were then used to determine a reliable and valid predictive model for the construct.

The knowledge domain of the Global Understanding Survey was most closely aligned with global awareness as defined in this study. The developers discussed two primary approaches to determining the content of test items. One approach centered on traditional international relations and area studies curricula and the other approach dealt with global issues that transcend nations and regions. The committee decided that the latter approach would facilitate development of items that were indicative of their impression of global understanding, as respondents would be able to apply knowledge from multiple disciplines and identify ramifications of issues across time, space, and social institutions. On the advice of a consulting faculty committee from Eisenhower College, items were developed to address 13 global themes: (a) environment; (b) food; (c) health; (d) energy; (e) religion; (f) arts and culture; (g) distribution of national characteristics; (h) relations among states; (i) war and armaments; (j) international monetary and trade arrangements; (k) human rights, (1) racial and ethnic issues; and (m) population. Test items addressed real-world issues and most could be answered correctly on the basis of knowledge gained from regular reading of newspapers with good international coverage. As would be noted from analysis of background correlates, students who reported regular news consumption scored higher on the test. Some items required background instruction or reading in geography, world history, economics, and international relations (Torney-Purta, 1982). 
The knowledge domain consisted of 101 multiple-choice questions. Items addressed international institutions, major historical events and trends, and legal and policy frameworks associated with the 13 global themes. The authors assessed the domain's reliability by analyzing the internal consistency of respondent scores. The authors contended that internal consistency was demonstrated through high reliability quotients ( .84 for freshmen, .86 for seniors, and .87 for two-year college students), meaning that performance on each item was consistent with performance on others. The authors did not expressly discuss evidence concerning the domain's validity. However, the authors did contend that low correlations between scores on the knowledge, affect, and language portions of the survey indicated that these domains were indeed distinct, which was consistent with their proposed construct for global understanding.

In his summary of the findings of the study, Barrows, et al. (1981) admitted that the project fell short of its purpose. Data analysis did not yield a strongly predictive model for global understanding and score interpretations did not provide any indications of methods for improving students' level of global understanding based on the fourcomponent construct. In particular, the authors found no relationship between students' educational experiences — coursework, language study, or study abroad — and their levels of knowledge. Barrows, et al. (1981) reported that the authors' disappointment in this finding was mitigated by their perception of the college experience as being deficient (e.g., fewer than 20 percent of students reported discussing global issues on a daily basis and more than ten percent claimed they had never had such discussions). Nevertheless, the lack of correlation between any sort of experience and performance on the knowledge 
section of the test shed considerable doubt concerning the utility of scores yielded, a major threat to construct validity (Messick, 1996).

Another likely threat to the validity of these scores was construct underrepresentation. Construct representation is traditionally achieved through cognitive task analysis and identification of constituent processes. It is substantiated by evidence that assessment tasks rely on knowledge and skills that are consistent with those required by the construct (Messick, 1996). Apart from the expert judge opinions of the Eisenhower Faculty committee for the knowledge domain, Barrows, et al. (1981) did not provide any theoretical or empirical evidence that would assist in the determination of construct representation. In fact, the opposite appears to be true; the authors stated in the introduction that it was hoped that through the exploration of potential components and possible correlates, the study might yield some suggestion of the nature of global understanding and how it is formed. On the basis of the data presented by Barrows, et al. (1981), it appears that the knowledge component of the Global Understanding Survey was too narrow and failed to address important aspects of the construct of global understanding that could be empirically linked to formal and/or informal educational experiences.

In addition, the items in this instrument did not align with the construct of global awareness as defined in this study. The items required students to demonstrate that they had knowledge of institutions and events that represented international and global connections, however the items did not require students to demonstrate understanding of how issues, trends, and systems influence each other to produce the globalized context in which citizens live and work. On the basis of the evidence provided by Barrows, et al. 
(1981), the Global Understanding Survey was a test of lower order recall of then-current world events, institutions, and legal and policy frameworks that were the manifestations of global interconnectedness. Data indicated a strong correlation between news consumption and test performance; however, the assessment did not present students with an authentic task such as the analysis of news articles. Although the assessment held students to a common criterion, it did not allow for differentiation of content across the curriculum, nor did it allow students to bring their diverse background knowledge to bear in their understanding of the items.

\section{Worldmindedness Scale}

The Worldmindedness Scale (Sampson \& Smith, 1957) was found to be one of the instruments most frequently used to measure a global awareness (Hett, 1993). It was composed of 32 items - 16 "worldminded" statements and 16 "non-worldminded" statements—addressing eight thematic dimensions: religion, immigration, government, economics, patriotism, race, education, and war. Participants expressed agreement or disagreement with each statement along a six-point Likert scale.

Sampson and Smith (1957) established the reliability of the scale through both odd-even and test-retest methods. They argued that the internal consistency of the frame of reference of the scale's items provided evidence of construct validity, as did correlation with a similar instrument, the Ethnocentrism Scale of the California Public Opinion Scale. Finally, Sampson and Smith employed the known-group technique to establish validity, by comparing the pretest/posttest difference in Worldmindedness Scale mean scores of students who travelled to Europe as tourists or with student organizations in the summer of 1950 with those of students who travelled to Europe with the Quaker 
International Voluntary Service. The authors established non-equivalency of the groups through two methods. First, selection for the Quaker service program was based on a requirement that participants possess a worldminded attitude, whereas this requirement did not exist for students travelling to Europe for other reasons. Second, pretest Worldminded Scale means differed significantly between the two groups, with Quaker service program students scoring higher than students travelling for other reasons.

In defining the construct upon which the instrument was based, Sampson and Smith (1957) distinguished between "international-mindedness," which referred to factual knowledge about international affairs, and "worldmindedness," which they defined as a "value orientation, or frame of reference, apart from knowledge about, or interest in, international relations" (p. 99). The authors described the worldminded individual as one concerned with a global rather than a nationalistic view of problems, whose primary affiliation was with all of humanity rather than a single national or cultural group. This orientation placed the Worldmindedness Scale within the global education conceptual framework. However, Sampson and Smith (1957) explicitly identified world-mindedness as an attitude rather than as a knowledge set. The items on the scale were worded in terms of the affective component of attitudes, for example, "It would be a dangerous procedure if every person in the world had equal rights which were guaranteed by an international charter" and "Our country is probably no better than many others" (p. 100). Furthermore, the authors' definition of worldmindedness did not refer to the concept of interrelatedness. Both of these issues called into question the construct validity of the Worldmindedness Scale as a measure of global awareness as defined in this study. 


\section{Global-mindedness Scale}

In the words of the late Jane Hett (1993), the Global-mindedness Scale was developed in order to "measure attitudes of students related to their sense of connection to, interest in, and responsibility for, the global community and the behaviors associated with this perspective" (p. 4). Hett intended for the instrument to be used in both curricular and co-curricular settings. Hett developed the concept of global-mindedness and her associated survey in response to several issues she perceived as calling into question the construct validity of the concept of worldmindedness and its associated survey. First, Hett claimed that worldmindedness did not incorporate a contemporary notion of diversity and that several of the items in the Sampson and Smith (1957) scale were overtly racist. She also noted that worldmindedness was not a truly global concept, in that it was defined in opposition to the concept of nationalism rather than subsuming it within its broader conceptual framework. As Hett noted, research did not indicate that a person could not be both patriotic and worldminded. The multitude of definitions in the literature for the concept of worldmindedness also concerned Hett, particularly in light of the contextual changes that had occurred in the years since the development of the term. Hett (1993) developed the concept of global-mindedness in order to address all of these concerns:

People who are global-minded possess an ecological world view, believe in the unity of humankind and the interdependence of humanity, support universal human rights, have loyalties that extend beyond national borders, and are futurists. (p. 9)

The final version of Hett's (1993) Global-mindedness Scale contained 30 items that participants scored for level of agreement on a five-point Likert scale. As with items 
in the Worldmindedness Scale, Hett's items addressed broad themes, although she argued that hers were more valid given her contemporary globalized context. These themes were: (a) interconnectedness of humanity; (b) cultural pluralism; (c) ethic of responsibility/care; (d) futurist orientation; and (e) behaviors.

Hett (1993) established validity through both qualitative and quantitative methods. Scale items were induced from both a review of literature and interviews with subjects who had demonstrated a commitment to understanding global-mindedness. Expert judges assessed the content validity of instrument drafts and validity was enhanced through revisions that addressed judges' comments and concerns. Hett also assessed content validity through instrument administration and scoring in a pilot study. Convergent validity was tested by establishing correlations between the Globalmindedness Scale and instruments that assessed related concepts such as the Chauvinism and International Understanding subscales of the Global Understanding Project instrument (Barrows, Ager, Bennett, Braun, Clark, Harris, \& Klein, as cited in Hett, 1993). In addition, Hett explored predictive validity among the demographic variables collected and mean Global-mindedness Scale scores. Reliability was established through tests of internal consistency.

The items in Hett's (1993) scale addressed beliefs and behaviors associated with the themes and her definition of global-mindedness. Some of these items addressed a global perspective as defined in this study, but only as indirect assessments of an individual's affective or behavioral inclination towards analyzing the world via multiple perspectives. Examples of indirect assessment of this skill included the items, "Americans can learn something of value from all different cultures;" "I enjoy trying to 
understand people's behavior in the context of their culture;" and, "I sometimes try to imagine how a person who is always hungry must feel” (pp. 193-195). There were no items in the scale that directly assessed the individual's ability to view the world from multiple perspectives.

\section{Intercultural Development Inventory}

This instrument was based on Bennett's (1986) Developmental Model of Intercultural Sensitivity (DMIS). The DMIS identified six orientations that explained how people construed cultural difference. It was theorized that as people move through these orientations, they develop greater degrees of intercultural competence, which was defined as the ability to think and act in interculturally appropriate ways (Hammer, Bennett, \& Wiseman, 2003). Bennett (1986) grounded his model in a constructivist view of meaning, asserting that the more perceptual and conceptual discriminations that could be brought to bear on one's experience of cultural difference, the more complex and nuanced one's understanding and behavioral reaction to the event could become.

Bennett (1986) posited that the following six cognitive schemata could be used to describe how cultural difference was understood:

Denial. Belief that one's own culture is the only real one. Other cultures are not understood or may be understood vaguely.

Defense. Belief that one's own culture is the only viable one. The world is divided into "us" and "them" and other cultures are deemed inferior.

Minimization. Belief that one's own culture is universal. Similarities may be played up and differences denied or deemed unimportant. 
Acceptance. Understanding that one's culture is one of a variety of cultures. One experiences difference but does not judge others as unequal.

Adaptation. Experience of other cultures' changes perceptions and behaviors appropriate to that culture. One's view of the world is expanded to include knowledge of other worldviews.

Integration. State in which one's experience of self includes movement in and out of different worldviews. Describes the experience of individuals who describe themselves as bicultural or multicultural.

Content validity for the IDI items was established by using statements gleaned from interviews with student volunteers from a private university in the United States. Expert judges provided feedback on the items in the draft pool. Revisions to the instrument were made on the basis of qualitative responses and agreement among expert judges of .60 or above. To establish construct validity, the developers correlated the IDI with two comparable instruments: the Worldmindedness Scale (Sampson \& Smith, 1957) and the Intercultural Anxiety Scale, a modified version of the Social Anxiety Scale (Gao \& Gudykunst, as cited in Hammer, Bennett, \& Wiseman, 2003). The developers explored predictive validity by exploring correlations between demographic variables and IDI scores. Reliability was established by both expert judge agreement as to the validity of the items and through a confirmatory factor analysis that established the goodness of fit between the items and the discrete dimensions of the DMIS model.

The final version of the Intercultural Development Inventory (IDI) included 50 items, ten of which addressed demographic variables. Items were composed of statements clustered around the six DMIS orientations, to which respondents expressed degrees of 
agreement or disagreement along a seven-point Likert scale. As with the Globalmindedness Scale, the IDI also presented items as statements of affect and behavioral inclination. Sample items such as, "People from other cultures are generally lazier compared to people from my culture," "People are the same despite outward differences in appearances," and "I often act as a cultural mediator in disagreements between people from different cultures" (Landis, Bennett, \& Bennett, 2004, p. 99) were indirect assessments of respondents' ability to view the world from others' perspectives.

\section{Global Perspective Inventory}

Braskamp, Braskamp, and Merrill (2009) developed the Global Perspective Inventory (GPI) to assess the holistic development of a global perspective. The developers based their instrument on two theoretical constructs: intercultural maturity and intercultural communication. They used the work of Kegan (as cited in Braskamp, Braskamp, and Merrill, 2009) and King and Magolda (as cited in Braskamp, Braskamp, and Merrill, 2009) to identify three major domains of human development—cognitive, intrapersonal, and interpersonal—and divided each domain into two scales. In each domain, one scale was based on intercultural development theory and the other was based on intercultural communication theory. The instrument's domains and scales are described as follows:

Cognitive domain. Knowledge and understanding of what one knows and judges important to know. Includes viewing knowledge as complex and taking into account multiple perspectives.

Knowing scale. Degree to which one views cultural context as important in judging what one knows and is important to know. 
Knowledge scale. Degree of understanding of other cultures and their influence on global society, as well as level of proficiency in more than one language.

Intrapersonal domain. Sense of self-direction, purpose, and awareness of values and strengths.

Identity scale. Degree of acceptance of the gender, racial, and ethnic components of one's identity.

Affect scale. Degree of acceptance of differing perspectives and emotional tolerance for complex situations.

Interpersonal domain. Willingness to interact with people who are different. Includes being able to view others differently and move from dependence to independence and interdependence.

Social responsibility scale. Degree of sense of interdependence and concern for others' well-being.

Social interactions scale. Degree of engagement with others who are different and sensitivity to difference.

The most recent version of the GPI at the time of this review (Version 6a) featured 69 items, 21 of which addressed demographic variables. Respondents expressed degree of agreement with item statements on a five-point Likert scale. In their discussion in the GPI manual of the psychometric characteristics of the instrument, the developers addressed the issue of trustworthiness of self-report responses. They wrote that certain characteristics of the testing situation made it less likely that a respondent would choose socially desirable answers, thus throwing into question the credibility of data gathered from the assessment. They claimed that respondents were more likely to be honest when 
the items were clearly understood and non-threatening and the results were not used for selection purposes.

The developers reported that they had used multiple methods to explore the valid use of scores yielded from the GPI. They established face validity for the survey by soliciting feedback from college students and experts in study abroad programs concerning the extent to which items were perceived as fair and reasonable. The developers claimed that this feedback led to revisions in the instrument, including reduction of non-demographic items from the 69 administered in the pilot test to the $40+$ administered in Version 5 and beyond. The focus of their exploration of face validity concerned the perceived utility of items for making decisions that affected campus climate and programs.

The developers also conducted several studies to explore construct validity. Intercorrelations between the two scales in each domain yielded what was deemed a "reasonable" relationship between them: $.18, .46$, and .42 . They reported that factor analyses supported the assertion that the survey provided a "reasonable" structure of the three domains of the construct. One study tested convergent validity by correlating the GPI with the 16 scales of the Inventory on Learning Climate and Student Well-Being (Walker, as cited in Chickering \& Braskamp, 2009). Twelve of the GPI items accounted for $47 \%$ of the variability of students' well-being and five of the items accounted for $20 \%$ of the variance. This study was based responses from a sample of 185 students attending a Catholic university in the east.

Further analyses revealed that male and female average responses varied among the scales, with the greatest difference being on the social responsibility scale. Mean 
scores also trended upward as students rose in class rank, with the greatest difference being between freshman and sophomore years. Students in private institutions tended to score higher than students in public institutions, and in a pre/post study of students in semester-long study abroad programs, differences in mean scores on five of the six scales were significantly different. The developers contended that these findings were consistent with those that would be expected given the instrument's theoretical construct.

The GPIs test-retest reliability and internal consistency were also reported. A study was conducted to explore the stability of pretest and posttest scores for two groups of students who spent time studying abroad: those who spent three weeks abroad and those who spent one semester abroad. In five of the six scales, scores were more stable in the group of students who spent only three weeks abroad, but the developers did not report the statistical significance of the difference between these groups. The developers reported the coefficient alpha reliabilities for each of the subscales, but they did not present a discussion of what these statistics revealed concerning the GPI's internal consistency.

The developers contended that these studies supported the valid use of GPI scores within the following educational contexts: (a) program or institutional interventions; (b) study abroad; (c) international student orientation; (d) service learning; (e) freshman-tosenior gains; (f) faculty perspectives; and/or (g) the accreditation and quality improvement process of Forum on Education Abroad. They emphasized that the GPI was not a criterion-based instrument and that its greatest utility was as a means of focusing discussion concerning students' holistic development as globally-oriented citizens. 
Of the assessments reviewed, the GPI featured items most closely aligned with this study's definition of global perspective; however, these items assessed the SLO indirectly. The majority of items aligned with global perspective were part of the Cognitive Knowing scale, the most straightforward item being, "I can evaluate issues from several different perspectives." This statement specifically required students to evaluate whether or not they are able to analyze problems from multiple perspectives. Other pertinent items include, "Cultural differences make me question what is really true" and a reverse-coded item, "I tend to judge the values of others based on my own value system" (Braskamp, et al., 2009). Although these items addressed respondents' multiperspective analytical ability, they were not direct assessments of the extent to which respondents possessed this skill.

\section{Summary}

This review of literature established the theoretical consistency of the major educational components of this study - global awareness, global perspective, and global learning-within the context of global education as a conceptual framework. Valid assessments of global awareness and global perspective must sample knowledge and cognitive processes that are theoretically consistent with these components (Messick, 1996). Based on this review, valid assessments of these outcomes must require that students engage in authentic problem solving activities concerning issues that transcend national borders and affect humans and their environment (Gibson, et al., 2008; Kniep, 1989; Roeper, 2008). The content of these real-world problems must be sufficiently complex as to require the application of multiple disciplinary perspectives and broadbased cultural, socio-political, scientific, and/or historical knowledge in their analysis and 
evaluation (Clarke, 2004; Kniep, 1989; Merryfield, 2008). Valid assessments must also require students to discern differentiated viewpoints that might be brought to bear in the problem's analysis and solution, as well as determine underlying influences of these viewpoints (Case, 1993; Hanvey, 1975). In summary, valid assessments of global awareness and global perspective must enable faculty and other stakeholders to gain insight into the knowledge and cognitive processes students utilize to determine responsible choices in a diverse and interconnected world.

This researcher was not able to find any existing assessment instruments that met these theoretical requirements. However, as a result of the review of development, reliability, and validity data provided by the instruments' developers, this researcher was able to identify methods that could provide useful data for those evaluating the meaningfulness and usefulness of scores yielded from the instrument designed for this study. These methods will be described in detail in Chapter 3 . 


\section{CHAPTER III}

\section{METHODS}

This chapter outlines the methods that were used in this study. This was a quasiexperimental study estimating the validity and reliability of scores yielded from rubrics developed to measure students' global awareness and global perspective. Chapter 3 describes the study's research questions and hypotheses, research design, variables, population, sample, instrumentation, data collection procedures, and data analysis procedures.

\section{Research Questions and Hypotheses}

This study addressed four research questions concerning the extent to which evidence supported the validity and reliability of scores yielded from rubrics measuring students' global awareness and global perspective. Research hypotheses were developed for each of the research questions:

\section{Research Question 1}

To what extent does evidence support the reliability of scores yielded from a rubric measuring students' global awareness?

Hypothesis 1. There will be a significant inter-rater agreement rate of at least .80 among raters using the global awareness rubric to measure the performance of students enrolled in a global learning course and among raters using the rubric to measure the performance of students who are not enrolled in a global learning course.

\section{Research Question 2}

To what extent does evidence support the reliability of scores yielded from a rubric measuring students' global perspective? 
Hypothesis 2. There will be a significant inter-rater agreement rate of at least .80 among raters using the global perspective rubric to measure the performance of students enrolled in a global learning course and among raters using the rubric to measure the performance of students who are not enrolled in a global learning course.

\section{Research Question 3}

To what extent does evidence support the validity of scores yielded from a rubric measuring students' global awareness?

Hypothesis $\mathbf{3}_{\mathbf{a}}$. There will be a significant difference on global awareness rubric scores between those students who are enrolled in a global learning course and those who are not when controlling for pretest scores and class status, independent of global perspective rubric scores.

Hypothesis $\mathbf{3}_{\mathbf{b}}$. There will be a significant difference on global awareness rubric scores between those students who are enrolled in a global learning course and those who are not when controlling for pretest scores and race/ethnicity, independent of global perspective rubric scores.

Hypothesis $\mathbf{3}_{\mathbf{c}}$. There will be a significant difference on global awareness rubric scores between those students who are enrolled in a global learning course and those who are not when controlling for pretest scores and fluency in more than one language, independent of global perspective rubric scores.

Hypothesis $\mathbf{3}_{\mathrm{d}}$. There will be a significant difference on global awareness rubric scores between those students who are enrolled in a global learning course and those who are not when controlling for pretest scores and time spent abroad, independent of global perspective rubric scores. 
Hypothesis $\mathbf{3}_{\mathbf{e}}$. There will be a significant difference on global awareness rubric scores between those students who are enrolled in a global learning course and those who are not when controlling for pretest scores and previous global learning course completion, independent of global perspective rubric scores.

Hypothesis $\mathbf{3}_{\mathbf{f}}$. There will be a significant difference on global awareness rubric scores between students who earn a "B" and above in a global learning course and students who earn a "D" and below in a global learning course.

\section{Research Question 4}

To what extent does evidence support the validity of scores yielded from a rubric measuring students' global perspective?

Hypothesis $\mathbf{4}_{\mathrm{a}}$. There will be a significant difference on global perspective rubric scores between those students who are enrolled in a global learning course and those who are not when controlling for pretest scores and class status, independent of global awareness rubric scores.

Hypothesis $\mathbf{4}_{\mathbf{b}}$. There will be a significant difference on global perspective rubric scores between those students who are enrolled in a global learning course and those who are not when controlling for pretest scores and race/ethnicity, independent of global awareness rubric scores.

Hypothesis $\mathbf{4}_{\mathrm{c}}$. There will be a significant difference on global perspective rubric scores between those students who are enrolled in a global learning course and those who are not when controlling for pretest scores and fluency in more than one language, independent of global awareness rubric scores. 
Hypothesis $\mathbf{4}_{\mathrm{d}}$. There will be a significant difference on global perspective rubric scores between those students who are enrolled in a global learning course and those who are not when controlling for pretest scores and time spent abroad, independent of global awareness rubric scores.

Hypothesis $\mathbf{4}_{\mathrm{e}}$. There will be a significant difference on global perspective rubric scores between those students who are enrolled in a global learning course and those who are not when controlling for pretest scores and previous global learning course completion, independent of global awareness rubric scores.

Hypothesis $\mathbf{4}_{\mathrm{f}}$. There will be a significant difference on global awareness rubric scores between students who earn a "B" and above in a global learning course and students who earn a "D" and below in a global learning course.

\section{Research Design}

The research design that was used in this study was quasi-experimental (Cook \& Campbell, 1979). Specifically, the study utilized a pretest/posttest nonequivalent group design to compare the average learning gains of students enrolled in global learning courses and students enrolled in non-global learning courses. This design was used in order to determine the extent to which evidence supported the construct validity and reliability of the rubrics. The study was designed to enable comparison of known groups: students who were enrolled in a global learning course and students who were not enrolled in a global learning course. An assumption of this study was that if students were exposed to global learning, they were more likely to develop global awareness and a global perspective. If this assumption held true, then valid rubrics would detect learning gain differences between groups pertaining to these outcomes. 
Participants were not randomly assigned to comparison groups because the study made use of existing classes of students. Selection bias is one of the primary threats to the internal validity of non-randomized quasi-experimental studies (Campbell \& Stanley, 1963; Cook \& Campbell, 1979). This study controlled for selection threat in two ways. The pretest was used to provide the researcher with some information concerning the equivalence of groups. The study also made use of statistical methods for controlling variation and isolating the primary independent variable (IV) and extraneous variables. These methods are explained below.

\section{Variables}

The primary IV in this study was completion of a global learning course. The dependent variables (DVs) in this study were student posttest scores on a rubric measuring global awareness and student posttest scores on a rubric measuring global perspective.

The study controlled for several extraneous variables. This was done in order to minimize within-group variance and maximize experimental variance. Controlling for these variables also minimized threats to the study's internal validity, as explained below.

\section{Class Status}

Students' class status was collected in order to control for maturation effects. Class status was coded as a continuous variable (“1,” freshman; "2," sophomore; "3," junior; "4," senior).

\section{Race/Ethnicity}

Students were asked to report their ethnicity: African-American; American Indian/Alaskan Native; Asian/Pacific Islander; European/White; Hispanic/Latino; or, 
Unknown/Other. In addition to these racial/ethnic categories, students were able to identify themselves as multi-ethnic. Multi-ethnic students may have a greater propensity for developing global awareness and global perspective than those who claim a single ethnicity (Banks, 2008; Moore, 2008). These data were used to control for selection bias. Race/ethnicity was coded as a categorical variable. Each variable was dummy coded, with " 1 " representing the self-reported race/ethnicity and " 0 " representing that a participant did not self-report that race/ethnicity.

\section{Fluency In More Than One Language}

Students were asked to report the number of languages they spoke fluently. Multilingual students may have a greater propensity for developing global awareness and global perspective than those who speak a single language (Clark, 1981; Tochon, 2009). These data were used to control for selection bias. Fluency in more than one language was coded as a continuous variable ("1," one language; "2," two languages; "3," three or more languages).

\section{Time Spent Abroad}

Students were asked to report the length of time they had spent abroad. Students who have travelled or lived abroad may have a greater propensity for developing global awareness and global perspective than those who have not (Donnelly-Smith, 2009; Sobania \& Braskamp, 2009). These data were used to control for selection bias. Time spent abroad was coded as a continuous variable (“0," no time spent abroad; " 1 ," two weeks or less spent abroad; “2,” more than two weeks spent abroad). 


\section{Previous Global Learning Course Completion}

Students were asked if they had previously completed a global learning course at the university. Previous completion of a global learning course might have affected the variability of pretest and posttest scores. Previous global learning course completion was dummy coded as a categorical variable (“1," previous global learning course completion; “0,” no previous global learning course completion).

\section{Global Learning Course Grade}

At the end of the semester, faculty members were asked to provide course grades for students who had completed the global learning courses. These data were collected in order to perform a within-group discriminant analysis for the purpose of determining whether there was a positive relationship between global learning course grades and global awareness and global perspective posttest scores. Students who earned a "B" or higher were coded "1;" students who earned a "D" or lower were coded " 0 ."

\section{Previous Case Response Assessment Completion}

Students were asked if they had previously taken the pretest. It was possible that some students may have previously taken the pretest as part of the university assessment program. Students who had previously taken the pretest were removed from the sample. This was done to control for testing bias.

\section{Population}

The population for this study was composed of ethnically and racially diverse undergraduate students enrolled in a large, Hispanic-serving, urban, public, research university in South Florida. Of the approximately 32,901 undergraduates enrolled in this institution at the time of the study, approximately $65 \%$ were of Hispanic/Latino origin, 
$12 \%$ were of European/White origin, 12\% were of Black or African American origin, 3\% were of Asian origin, 1\% were of American Indian or Alaskan Native origin, and .05\% were of Native Hawaiian or other Pacific Islander origin. Approximately 93\% of undergraduates were classified as in-state residents, $4 \%$ as out-of-state residents, and 3\% as international students.

Approximately $64 \%$ of the undergraduate student body was composed of students who had transferred from another institution to complete their baccalaureate degree. Annually approximately $2 \%$ of undergraduates engaged in credit-bearing travel abroad programs and $1 \%$ engaged in non-credit bearing travel abroad, including service and internships. As a public institution, the university had a foreign language requirement for entrance. All students were required to complete at least two years of the same foreign language for admission. The College of Arts and Sciences had an additional foreign language requirement. This entrance requirement stated that students needed to demonstrate proficiency equivalent to the second semester university level in a foreign language. The university did not collect data on the number of undergraduates who reported fluency in more than one language.

\section{Sample}

This study involved a purposive sample of students enrolled in two global learning courses and students enrolled in two non-global learning courses. Students enrolled in the global learning courses were considered the treatment group. Students enrolled in the non-global learning courses were considered the control group. The sample size for the study was sought on the basis of a power analysis. Power refers to the probability that a false null hypothesis will be rejected (i.e. the probability of not 
committing a Type II error). One of the ways the researcher can increase power is to increase sample size. Large sample sizes more accurately reflect the characteristics of the population, thus increasing power and reducing Type II error. Since it is often impractical to obtain large samples in real-world settings, researchers rely on sample size rules of thumb in order to achieve maximum power in the context of available resources (Green, 1991; Van Voorhis \& Morgan, 2007).

Based upon the maximum number of linearly independent vectors in the models tested, a power analysis was conducted. The analysis indicated that for an alpha level of .05 and a medium effect size, $f^{2}=.15$ (Cohen, 1988), the power would be at least .99 for a sample size of at least 400 .

\section{Instrumentation}

This researcher initially drafted a performance assessment instrument in spring 2009 to assess the six global learning SLOs that the university was working with at that time. The draft instrument was composed of two parallel case studies (see Appendices C and D), open-ended questions concerning each of the case studies, and an analytic scoring rubric that addressed all six of the outcomes (see Appendix E). The scoring criteria in the draft rubric were generated from input from six faculty members who were field testing the use of the global learning SLOs to revise existing courses across the general education curriculum during spring 2009. The criteria reflected faculty members' expectations as well as observed student response trends during in-person and online discussions and other active learning and performance-based assessment activities. The rubrics were also developed according to best practices described in the research literature. The analytic rubric contained no more than five scoring levels, and descriptive 
performance criteria were included to assist raters in distinguishing qualitative response differences pertaining to each score level (Popham, 1997). The faculty members field tested the parallel forms of the draft instrument and provided feedback to the researcher. Late in the spring 2009 semester the university narrowed the knowledge and skill outcomes to two, global awareness and global perspective. Based on faculty feedback concerning the draft analytic rubric and the constructs underlying the revised SLOs, separate global awareness and global perspective rubrics were drafted for use with each of the cases and their corresponding open-ended questions. At this point, benchmark responses derived from the pilot studies were included in the rubrics to operationalize the scoring criteria (Popp, Ryan, \& Thompson, 2009) (see Appendices F and G).

To evaluate the construct validity of the revised parallel case narratives, questions, and scoring rubrics, this researcher conducted a web-based survey of the faculty members involved in the spring 2009 field tests (see Appendix H). The instrument forms were also emailed to three outside global learning experts for feedback (see Appendix I). Both sets of survey results were used to make further adjustments to the case narratives, questions, and rubrics.

Field tests of the newly revised parallel forms of the instrument were conducted in fall 2009 and spring 2010. Feedback from raters and faculty testing the instruments was used to make further revisions to the wording of the criteria. This was done to enhance construct validity and inter-rater agreement. In fall 2010, the university began delivering the final forms of the instrument to $10 \%$ samples of incoming freshman, transfer students, and graduating seniors. These final forms were also used in this study. In the final forms, for each of the parallel cases one open-ended question addressed the global awareness 
SLO and the other addressed the global perspective SLO. Two five-level (“0”-“4”) holistic rubrics were used to score students' responses according to their level of achievement of the SLOs (see Appendices J and K). The levels of the rubric corresponded to the levels of Bloom's (1956) Taxonomy of Cognitive Development. A score of " 3 " represented the cognitive level of analysis. Faculty and raters involved in the instruments' field tests agreed that Bloom's Taxonomy reflected the underlying cognitive processes involved in the performance assessment of global awareness and global perspective. They also agreed that the cognitive level of analysis was a meaningful academic criterion and a meaningful minimum criterion for success.

\section{Data Collection Procedures}

To gather participants, the researcher identified global learning and non-global learning courses being taught in the same areas of the general education curriculum that covered similar subject matter. Emails were sent to faculty teaching the selected global learning foundations courses to introduce the study and to request cooperation. Emails were also sent to chairs of departments that oversaw the selected non-global learning courses to introduce the study and to request cooperation in identifying faculty teaching class sections in which the pretest and posttest could be administered (see Appendix L). Of the participating classes, one global learning course and one non-global learning course were in the "Humanities with Writing" section of the general education curriculum and one global learning course and one non-global learning course were in the "Arts" section of the general education curriculum. Both of the courses in the "Arts" section were survey courses rather than fine or performing arts courses. 
The pretest was administered in class during the first two weeks of the semester (see Appendix M). The posttest was administered in class during the last two weeks of the semester (see Appendix N). Faculty members were informed that the test administration would take approximately 45 minutes. To encourage students to give the assessment their best effort, faculty members were asked to remain present during the test administration and to assign some sort of grade credit for completion of the task. The researcher attended class to introduce and administer the assessments. To protect confidentiality, student names were not collected and only student IDs were placed on the assessment forms. The completed assessments were kept in a locked file in the researcher's office; only the researcher, raters, and participating faculty members had access to the completed assessments.

Data concerning students' class status, race/ethnicity, language fluency, time spent abroad, previous global learning course completion, and previous assessment completion were also self-reported on the assessment forms (see Appendices $\mathrm{M}$ and $\mathrm{N}$ ). At the end of the semester, faculty members were asked to provide course grades for students who had completed the global learning courses. These data were collected in order to perform a within-group discriminant analysis, described below.

Trained faculty raters scored the completed assessments. Faculty raters received a stipend to score the assessments from this study as well as those collected as part of the university's pre/post assessment program. Two raters read and scored student responses to each question. Each rater assigned each response a score (“0"-“4”). If the two raters' scores were not discrepant, the final response score was an average of the two raters' scores. Discrepancy was defined as a difference of more than one point between the two 
raters' scores. For instance, if one rater gave a response a score of " 1 " and a second rater gave the same response a score of " 3 ," the scores were considered discrepant. In the case of discrepancy, a third trained rater read the response and the final score was an average of the three raters' scores.

Prior to the scoring sessions, raters were sent a letter outlining the scoring procedures, as well as copies of the cases, questions, and rubrics for their review (see Appendix O). At the beginning of the scoring sessions, the team of raters participated in a 90-minute training. The training consisted of an open discussion reviewing the cases, questions, and rubrics, followed by a norming session in which all raters scored and discussed anchor papers. The researcher chose anchor papers from the sample of student responses. Each anchor paper was chosen because it exemplified criteria described at each of the rubrics' scoring levels. The norming session began with raters silently reading an anchor paper that the researcher had determined exemplary of a score of " 3 ." Raters were not informed of the researcher's score determination for the anchor paper. Raters were each provided with a packet of 5 score signs (“0”-“4”); after all raters finished reading the anchor paper, they simultaneously held up the sign corresponding to the score they assigned the paper. Raters discussed each other's responses, with raters being asked to cite specific text in the anchor paper that influenced their score determination. The session continued with raters repeating the same process for anchor papers exemplifying scores of " 0, , "1," "2," and "4." After the norming session, the training continued with a sample scoring session ( $10 \%$ of the total papers to be scored) to establish an inter-rater agreement rate of at least .80 . 


\section{Data Analysis Procedures}

Reliability in this study was operationally defined as the percentage of inter-rater agreement. Statistically significant inter-rater agreement of at least .80 was deemed necessary to meet the minimum reliability requirement.

Linear regression (Cohen, Cohen, West, \& Aiken, 2003; McNeil, et al., 2012) was used to test the research hypotheses associated with research questions 3 and 4. Linear regression is the general case of the least square solution. Analysis of variance (ANOVA) and analysis of covariance (ANCOVA) are subsets of the general linear model. Since this study was conducted to determine if rubric scores predicted known groups, independent of the number of covariates, ANCOVA was determined an appropriate approach to use. ANCOVA is a general linear model with a continuous DV and two or more IVs. For ANCOVA, covariates may be categorical or continuous. Since these conditions matched those of this study, ANCOVA was further determined an appropriate statistical procedure. Post hoc analyses were also conducted in order to determine if significant interaction existed among predictor variables in the multiple regression models.

One-tailed tests of significance at the .05 level were used to test the research hypotheses because there was reason to believe that the treatment group would do better than the control group. Performing directional tests increased the study's power (i.e., detecting if a relationship exists). Similarly, a within-group analysis was performed to determine if a positive relationship existed between rubric scores and students' grades in global learning courses. 


\section{CHAPTER IV}

\section{RESULTS}

The purpose of this study was to demonstrate the extent to which evidence supported the validity and reliability of scores yielded from rubrics designed to measure undergraduate students' global awareness and global perspective. Data were collected and analyzed in order to test the research hypotheses associated with the study's four research questions:

1. To what extent does evidence support the reliability of scores yielded from a rubric measuring students' global awareness?

2. To what extent does evidence support the reliability of scores yielded from a rubric measuring students' global perspective?

3. To what extent does evidence support the validity of scores yielded from a rubric measuring students' global awareness?

4. To what extent does evidence support the validity of scores yielded from a rubric measuring students' global perspective?

This chapter presents a description and analysis of the data collected to test each of the research hypotheses, including post hoc analyses. The chapter includes an explanation of how the sample was narrowed, a description of the participants, analysis of the test results for each of the study's research hypotheses, and a summary of findings.

\section{Narrowing the Sample}

For this study, a total of 306 students took the pretest. Of students taking the pretest, 181 students (59\%) were enrolled in a global learning course and 125 students (41\%) were enrolled in a non-global learning course. A total of 257 students took the 
posttest. Of students taking the posttest, 149 students (58\%) were enrolled in a global learning course and 108 students (42\%) were enrolled in a non-global learning course. Students' pretest scores were used to establish equivalence between groups. An independent samples $t$-test was conducted to compare pretest mean scores between students enrolled in a global learning course and students enrolled in a non-global learning course. There was a significant difference in mean global awareness pretest scores between students enrolled in a global learning course $(M=1.49, S D=.80)$ and students enrolled in a non-global learning course $(M=1.76, S D=.89) ; t(304)=2.84$, $p=.005$. There was also a significant difference in mean global perspective pretest scores between students enrolled in a global learning course $(M=.92, S D=.69)$ and students enrolled in a non-global learning course $(M=1.16, S D=.84) ; t(304)=2.73, p=.007$. Since pretest score means were found to be significantly different, the study sample was narrowed to include only those students who took both the pretest and the posttest. The final matched-pair sample was composed of a total of 220 students, with 132 students $(60 \%)$ enrolled in a global learning course and 88 students $(40 \%)$ enrolled in a non-global learning course.

Based on the new sample size, an analysis was conducted to determine the study's power. The analysis indicated that for an alpha level of .05 and a medium effect size, $f^{2}=.15$ (Cohen, 1988), the power would be at least .99 for a sample of 220 .

\section{Description of Participants}

The first page of the assessment form contained 10 questions requesting background information from each student. Tables 1 through 5 present the demographic characteristics of the study's participants and frequency analyses. Table 6 presents a 
frequency analysis of final grades for students enrolled in a global learning course. These data were collected after students completed the posttest and the semester was over.

\section{Class Status}

Students who participated in the study were asked to report their class status. Table 1 shows that 92 students (69.7\%) enrolled in a global learning course and 30 students (34.1\%) enrolled in a non-global learning course were freshmen; 19 students (14.4\%) enrolled in a global learning course and 35 students (39.8\%) enrolled in a nonglobal learning course were sophomores; 13 students $(9.8 \%)$ enrolled in a global learning course and 15 students (17\%) enrolled in a non-global learning course were juniors; and, 8 students $(6.1 \%)$ enrolled in a global learning course and 8 students $(9.1 \%)$ enrolled in a non-global learning course were seniors. Crosstabulation analysis determined that the groups differed significantly in terms of students' class status, Pearson $\chi^{2}(3, N=220)=$ $28.741, p<.0001$.

Table 1

Descriptive Statistics of the Student Sample, Class Status

\begin{tabular}{lccccc}
\hline \multirow{2}{*}{ Class Status } & \multicolumn{2}{c}{ Global Learning } & & \multicolumn{2}{c}{ Non-Global Learning } \\
\cline { 2 - 3 } \cline { 5 - 6 } Freshman & $N$ & $\%$ & & $N$ & $\%$ \\
Sophomore & 92 & 69.7 & & 30 & 34.1 \\
Junior & 19 & 14.4 & & 35 & 39.8 \\
Senior & 13 & 9.8 & & 15 & 17.0 \\
\hline
\end{tabular}

\section{Race/Ethnicity}

Participants were also asked to report their race/ethnicity. Table 2 shows that as in the general university population, the majority of participants were Hispanic/Latino 
(59.1\% of students enrolled in a global learning course and $47.7 \%$ of students enrolled in a non-global learning course). Also as in the university population, European/White students and African American students composed nearly the same proportion of the sample. Of students enrolled in a global learning course, $16 \%$ were European/White and 17\% were African American; of students enrolled in a non-global learning course, 16\% were European/White and 15\% were African American. Crosstabulation analysis determined that the groups were similar across all races/ethnicities, including Hispanic/Latino, Pearson $\chi^{2}(1, N=220)=2.750, p=.064$.

Table 2

Descriptive Statistics of the Student Sample, Race/Ethnicity

\begin{tabular}{lccccc}
\hline & \multicolumn{2}{c}{ Global Learning } & & \multicolumn{2}{c}{ Non-Global Learning } \\
\cline { 2 - 3 } \cline { 5 - 6 } Race/Ethnicity & $N$ & $\%$ & & $N$ & $\%$ \\
\hline African American & 17 & 12.9 & & 15 & 17.0 \\
American & 0 & 0 & & 0 & 0 \\
Indian/Alaskan Native & & & & & 4.5 \\
Asian/Pacific Islander & 7 & 5.3 & & 4 & 18.2 \\
European/White & 16 & 12.1 & & 16 & 47.7 \\
Hispanic/Latino & 78 & 59.1 & & 42 & 5.7 \\
Multiple Ethnicities & 11 & 8.3 & & 5 & 6.8 \\
Unknown/Other & 3 & 2.3 & & 6 & \\
\hline
\end{tabular}

\section{Fluency In More Than One Language}

Table 3 shows that the majority of participants in both global learning courses (73.5\%) and non-global learning courses (69.4\%) spoke two or more languages. The university did not maintain statistics on the number of languages students spoke fluently. 
In terms of the number of languages students reported speaking fluently, the groups were similar, Pearson $\chi^{2}(2, N=220)=1.691, p=.429$.

Table 3

Descriptive Statistics of the Student Sample, Fluency in More Than One Language

\begin{tabular}{lcccccc}
\hline & Fluency & \multicolumn{2}{c}{ Global Learning } & & \multicolumn{2}{c}{ Non-Global Learning } \\
\cline { 2 - 3 } \cline { 5 - 6 } & & $N$ & $\%$ & & $N$ & $\%$ \\
\hline One & 35 & 26.5 & & 27 & 30.7 \\
Two & 87 & 65.9 & & 51 & 58.0 \\
Three or more & 10 & 7.6 & & 10 & 11.4 \\
\hline
\end{tabular}

\section{Time Spent Abroad}

Participants in the study were asked to report the amount of time they had spent abroad. Table 4 shows that slightly less than the majority of students in both global learning courses $(47.7 \%)$ and non-global learning courses $(48 \%)$ had spent more than two weeks abroad. The groups were very similar concerning the amount of time students' had spent abroad, Pearson $\chi^{2}(2, N=220)=.198, p=.906$.

Table 4

Descriptive Statistics of the Student Sample, Time Spent Abroad

\begin{tabular}{lccccc}
\hline \multirow{2}{*}{ Time Spent Abroad } & \multicolumn{2}{c}{ Global Learning } & & \multicolumn{2}{c}{ Non-Global Learning } \\
\cline { 2 - 3 } \cline { 5 - 6 } None & $N$ & $\%$ & & $N$ & $\%$ \\
Two weeks or less & 31 & 23.5 & & 22 & 25.0 \\
More than two weeks & 38 & 28.8 & & 23 & 26.1 \\
\hline
\end{tabular}

\section{Previous Global Learning Course Completion}

Table 5 shows that the majority of students enrolled in a global learning course (70.5\%) and of students enrolled in a non-global learning course $(76.1 \%)$ had not 
previously taken a global learning course. In terms of the number of students who had previously taken a global learning course, the groups were similar, Pearson $\chi^{2}(1, N=$ $220)=.859, p=.221$.

Table 5

Descriptive Statistics of the Student Sample, Previous Global Learning Course

\begin{tabular}{lcccccc}
\hline & \multicolumn{2}{c}{ Global Learning } & & \multicolumn{2}{c}{ Non-Global Learning } \\
\cline { 2 - 3 } \cline { 5 - 6 } & Previous Course & $N$ & $\%$ & & $N$ & $\%$ \\
\hline Yes & 39 & 29.5 & & 21 & 23.9 \\
No & 93 & 70.5 & & 67 & 76.1 \\
\hline
\end{tabular}

\section{Global Learning Course Grade}

After the end of the semester, faculty teaching global learning courses were asked to report their students' grades in order to perform a within-group discriminant analysis. This was done to determine whether there was a positive relationship between global learning course grades and global awareness and global perspective posttest scores. Table 6 shows the course grades for students enrolled in a global learning course.

Table 6

Descriptive Statistics of the Student Sample, Global Learning Course Grade

\begin{tabular}{lcc}
\hline Global Learning Course Grade & $N$ & $\%$ \\
\hline "B" or higher & 97 & 84.3 \\
"D" or lower & 18 & 15.7 \\
\hline
\end{tabular}

\section{Data Analysis}

The results of tests for each of the study's hypotheses are reported in this section. The percentage of inter-rater agreement was calculated to test the hypotheses associated with research questions 1 and 2 . Linear regression analysis was used to test the hypotheses associated with research questions 3 and 4 . For hypotheses tested with 
multiple linear regression, the full regression model was tested against the restricted model in order to determine if the primary IV, the global learning course, accounted for a significant amount of the unique variance in predicting each DV, global awareness and global perspective posttest scores, independent of the covariates. For hypotheses tested with simple linear regression, the full regression model is presented. Post hoc analyses were conducted in order to determine if significant interaction existed among predictor variables in the multiple regression models.

\section{Research Question 1}

To what extent does evidence support the reliability of scores yielded from a rubric measuring students' global awareness?

\section{Hypothesis 1}

The first hypothesis stated that there would be significant inter-rater agreement of at least .80 among raters who used the global awareness rubric to measure the performance of students enrolled in a global learning course and among raters who used the rubric to measure the performance of students who were not enrolled in a global learning course. There was $89 \%$ agreement and $11 \%$ disagreement among raters using the global awareness rubric to score all students' pretests. A chi-square test of frequency found that these results were significant, $\chi 2(1, N=220)=65.68, p<.0001$. There was 95\% agreement and 5\% disagreement among raters using the global awareness rubric to score all students' posttests. A chi-square test of frequency that these results were also significant, $\chi 2(1, N=220)=174.62, p<.0001$. The results of these tests confirmed research hypothesis 1 . 


\section{Research Question 2}

To what extent does evidence support the reliability of scores yielded from a rubric measuring students' global perspective?

\section{Hypothesis 2}

The second hypothesis stated that there would be significant inter-rater agreement of at least .80 among raters who used the global perspective rubric to measure the performance of students enrolled in a global learning course and among raters who used the rubric to measure the performance of students who were not enrolled in a global learning course. There was $92 \%$ agreement and $8 \%$ disagreement among raters using the global perspective rubric to score all students' pretests. A chi-square test of frequency found that these results were significant, $\chi 2(1, N=220)=157.25, p<.0001$. There was $91 \%$ agreement and $9 \%$ disagreement among raters using the global perspective rubric to score all students' posttests. A chi-square test of frequency found that these results were also significant, $\chi 2(1, N=220)=150.56, p<.0001$. The results of these tests confirmed research hypothesis 2 .

\section{Research Question 3}

To what extent does evidence support the validity of scores yielded from a rubric measuring students' global awareness?

Hypothesis $\mathbf{3}_{\mathrm{a}}$. This hypothesis stated that there would be a significant difference on global awareness posttest scores between those students who were enrolled in a global learning course and those who were not when controlling for global awareness pretest scores and class status, independent of global perspective posttest scores. A multiple regression analysis was conducted to test this hypothesis using the following models: 
- Full model: $\mathrm{y}$ (Global Awareness Posttest Score $)=\mathrm{a}_{0} \mathrm{u}+\mathrm{a}_{1}$ (Global Learning Course Enrollment $)+\mathrm{a}_{2}($ Global Awareness Pretest Score $)+\mathrm{a}_{3}($ Status $)+$ $\mathrm{a}_{4}($ Global Perspective Posttest Score $)+\mathrm{E}_{1}$

- Restricted model: y (Global Awareness Posttest Score $)=a_{0} u+a_{5}$ (Global Awareness Pretest Score $)+a_{6}($ Status $)+a_{7}($ Global Perspective Posttest Score $)+$ $\mathrm{E}_{2}$

A summary of results of this analysis is presented in Table 7. The coefficients and significance of each of the models' variables are presented in Table 8 .

Table 7

Hypothesis $3_{a}$ : Summary of Results of Regression Analysis

\begin{tabular}{lccccc}
\hline \multicolumn{1}{c}{ Model } & $R^{2}$ & $d f$ & $F$ Change & $p$ & Significance \\
\hline Full & .236 & 4 & .312 & .577 & NS \\
Restricted & .235 & 3 & & & \\
\hline
\end{tabular}

Table 8

Hypothesis $3_{a}$ : Predictor Variables to Determine Global Awareness Posttest Scores

\begin{tabular}{|c|c|c|c|c|c|c|}
\hline \multirow[b]{2}{*}{ Variables } & \multicolumn{3}{|c|}{ Full Model } & \multicolumn{3}{|c|}{ Restricted Model } \\
\hline & $b$ & $t$ & $p$ & $b$ & $t$ & $p$ \\
\hline (Constant) & .787 & 4.488 & .000 & .730 & 5.108 & .000 \\
\hline Global Awareness & & & & & & \\
\hline Pretest Score & .129 & 2.227 & .027 & 136 & 2.412 & .017 \\
\hline Status & 019 & .371 & .711 & .026 & .535 & .593 \\
\hline $\begin{array}{l}\text { Global Perspective } \\
\text { Posttest Score }\end{array}$ & .438 & 7.433 & .000 & 437 & 7.425 & .000 \\
\hline $\begin{array}{l}\text { Global Learning } \\
\text { Course Enrollment }\end{array}$ & -.056 & -.558 & .577 & & & \\
\hline
\end{tabular}

Note. The DV in this analysis was students' global awareness posttest scores. See Chapter 3 for a description and coding of other variables. 
These results indicated that the global learning course did not account for a significant amount of the unique variance in predicting global awareness posttest scores, independent of the global awareness pretest score, class status, and the global perspective posttest score $(p=.577, \alpha<.05)$. These results did not confirm research hypothesis $3_{\mathrm{a}}$.

Hypothesis $\mathbf{3}_{\mathbf{b}}$. This hypothesis stated that there would be a significant difference on global awareness posttest scores between those students who were enrolled in a global learning course and those who were not when controlling for global awareness pretest scores and race/ethnicity, independent of global perspective posttest scores. A multiple regression analysis was conducted to test this hypothesis using the following models:

- Full model: y (Global Awareness Posttest Score) $=\mathrm{a}_{0} \mathrm{u}+\mathrm{a}_{8}$ (Global Learning Course Enrollment $)+\mathrm{a}_{9}($ Global Awareness Pretest Score $)+\mathrm{a}_{10}($ EurWhite $)+$ $\mathrm{a}_{11}($ AsnPacI $)+\mathrm{a}_{12}($ HispLat $)+\mathrm{a}_{13}($ Mult $)+\mathrm{a}_{14}($ UnkOther $)+\mathrm{a}_{15}($ Global Perspective Posttest Score) $+\mathrm{E}_{3}$

- Restricted model: y (Global Awareness Posttest Score $)=\mathrm{a}_{0} \mathrm{u}+\mathrm{a}_{16}($ Global Awareness Pretest Score $)+a_{17}($ EurWhite $)+a_{18}($ AsnPacI $)+a_{19}($ HispLat $)+$ $\mathrm{a}_{20}($ Mult $)+\mathrm{a}_{21}($ UnkOther $)+\mathrm{a}_{22}($ Global Perspective Posttest Score $)+\mathrm{E}_{4}$ A summary of results of this analysis is presented in Table 9 . These results indicated that the global learning course did not account for a significant amount of the unique variance in predicting global awareness posttest scores, independent of the global awareness pretest score, race/ethnicity, and the global perspective posttest score ( $p=$ $.338, \alpha<.05)$. These results did not confirm research hypothesis $3_{\mathrm{b}}$. Coefficients and significance of each of the models' variables are presented in Table 10. 
Table 9

Hypothesis $3_{b}$ : Summary of Results of Regression Analysis

\begin{tabular}{lccccc}
\hline Model & $R^{2}$ & $d f$ & $F$ Change & $p$ & Significance \\
\hline Full & .248 & 8 & .921 & .338 & NS \\
Restricted & .245 & 7 & & & \\
\hline
\end{tabular}

Table 10

Hypothesis $3_{b}$ : Predictor Variables to Determine Global Awareness Posttest Scores

\begin{tabular}{|c|c|c|c|c|c|c|}
\hline \multirow[t]{2}{*}{ Variables } & \multicolumn{3}{|c|}{ Full Model } & \multicolumn{3}{|c|}{ Restricted Model } \\
\hline & $b$ & $t$ & $p$ & $b$ & $t$ & $p$ \\
\hline (Constant) & .865 & 5.028 & .000 & .797 & 5.084 & .000 \\
\hline Global Awareness & & & & & & \\
\hline Pretest Score & .113 & 1.937 & .054 & .125 & 2.206 & .028 \\
\hline European/White & -.058 & -.332 & .740 & -.054 & -.311 & .756 \\
\hline Asian/Pacific Islander & -.124 & -.511 & .610 & -.132 & -.543 & .588 \\
\hline Hispanic/Latino & .073 & .523 & .601 & .061 & .441 & .660 \\
\hline Multiple Ethnicities & -.024 & -.112 & .911 & -.038 & -.179 & .858 \\
\hline Unknown/Other & -.321 & -1.223 & .223 & -.300 & -1.145 & .254 \\
\hline $\begin{array}{l}\text { Global Perspective } \\
\text { Posttest Score }\end{array}$ & .431 & 7.253 & .000 & .428 & 7.206 & .000 \\
\hline $\begin{array}{l}\text { Global Learning } \\
\text { Course Enrollment }\end{array}$ & -.095 & -.959 & .338 & & & \\
\hline
\end{tabular}

Note. No participants reported American Indian/Alaskan Native race/ethnicity, therefore this variable was excluded from analysis. The variable African American was excluded as a constant. The DV in this analysis was students' global awareness posttest scores. See Chapter 3 for a description and coding of other variables.

Hypothesis $3_{c}$. This hypothesis stated that there would be a significant difference on global awareness posttest scores between those students who were enrolled in a global learning course and those who were not when controlling for global awareness pretest scores and fluency in more than one language, independent of global perspective posttest 
scores. A multiple regression analysis was conducted to test this hypothesis using the following models:

- Full model: y (Global Awareness Posttest Score) $=\mathrm{a}_{\mathrm{o}} \mathrm{u}+\mathrm{a}_{23}$ (Global Learning Course Enrollment $)+\mathrm{a}_{24}($ Global Awareness Pretest Score $)+\mathrm{a}_{25}($ Language $)+$ $\mathrm{a}_{26}($ Global Perspective Posttest Score $)+\mathrm{E}_{5}$

- Restricted model: y (Global Awareness Posttest Score $)=a_{0} u+a_{27}($ Global Awareness Pretest Score $)+\mathrm{a}_{28}($ Language $)+\mathrm{a}_{29}($ Global Perspective Posttest Score) $+\mathrm{E}_{6}$ A summary of results of this analysis is presented in Table 11. These results indicated that the global learning course did not account for a significant amount of the unique variance in predicting global awareness posttest scores, independent of the global awareness pretest score, fluency in more than one language, and the global perspective posttest score $(p=.499, \alpha<.05)$. These results did not confirm research hypothesis $3_{\mathrm{c}}$. The coefficients and significance of each of the models' variables are presented in Table 12.

Table 11

Hypothesis $3_{c}$ : Summary of Results of Regression Analysis

\begin{tabular}{lccccc}
\hline \multicolumn{1}{c}{ Model } & $R^{2}$ & $d f$ & $F$ Change & $p$ & Significance \\
\hline Full & .237 & 4 & .460 & .499 & NS \\
Restricted & .236 & 3 & & & \\
\hline
\end{tabular}


Table 12

Hypothesis $3_{c}$ : Predictor Variables to Determine Global Awareness Posttest Scores

\begin{tabular}{|c|c|c|c|c|c|c|}
\hline \multirow[b]{2}{*}{ Variables } & \multicolumn{3}{|c|}{ Full Model } & \multicolumn{3}{|c|}{ Restricted Model } \\
\hline & $b$ & $t$ & $p$ & $b$ & $t$ & $p$ \\
\hline (Constant) & .711 & 3.501 & .001 & .661 & 3.500 & .001 \\
\hline Global Awareness & & & & & & \\
\hline Pretest Score & .129 & 2.235 & .026 & .137 & 2.432 & .016 \\
\hline Language & .062 & .770 & .442 & .062 & .772 & .441 \\
\hline Global Perspective & & & & & & \\
\hline Posttest Score & .441 & 7.468 & .000 & .438 & 7.448 & .000 \\
\hline Global Learning & & & & & & \\
\hline Course Enrollment & -.066 & -.678 & .499 & & & \\
\hline
\end{tabular}

Note. The DV in this analysis was students' global awareness posttest scores. See Chapter 3 for a description and coding of other variables.

Hypothesis $\mathbf{3}_{\mathrm{d}}$. This hypothesis stated that there would be a significant difference on global awareness posttest scores between those students who were enrolled in a global learning course and those who were not when controlling for global awareness pretest scores and time spent abroad, independent of global perspective posttest scores. A multiple regression analysis was conducted to test this hypothesis using the following models:

- Full model: y (Global Awareness Posttest Score $)=\mathrm{a}_{\mathrm{o}} \mathrm{u}+\mathrm{a}_{30}($ Global Learning Course Enrollment $)+\mathrm{a}_{31}($ Global Awareness Pretest Score $)+\mathrm{a}_{32}($ Abroad $)+$ $\mathrm{a}_{33}$ (Global Perspective Posttest Score $)+\mathrm{E}_{7}$

- Restricted model: y (Global Awareness Posttest Score $)=\mathrm{a}_{\mathrm{o}} \mathrm{u}+\mathrm{a}_{34}($ Global Awareness Pretest Score $)+\mathrm{a}_{35}$ (Abroad) $+\mathrm{a}_{36}$ (Global Perspective Posttest Score) $+\mathrm{E}_{8}$ 
A summary of results of this analysis is presented in Table 13. These results indicated that the global learning course did not account for a significant amount of the unique variance in predicting global awareness posttest scores, independent of the global awareness pretest score, time spent abroad, and the global perspective posttest score $(p=.500, \alpha<.05)$. These results did not confirm research hypothesis $3_{\mathbf{d}}$. The coefficients and significance of each of the models' variables are presented in Table 14.

Table 13

Hypothesis $3_{d}$ : Summary of Results of Regression Analysis

\begin{tabular}{lccccc}
\hline \multicolumn{1}{c}{ Model } & $R^{2}$ & $d f$ & $F$ Change & $p$ & Significance \\
\hline Full & .236 & 4 & .456 & .500 & NS \\
Restricted & .234 & 3 & & & \\
\hline
\end{tabular}

Table 14

Hypothesis $3_{\text {d: }}$ Predictor Variables to Determine Global Awareness Posttest Scores

\begin{tabular}{lccccccc}
\hline & \multicolumn{3}{c}{ Full Model } & & \multicolumn{3}{c}{ Restricted Model } \\
\cline { 2 - 3 } \cline { 6 - 7 } Variables & $b$ & $t$ & $p$ & & $b$ & $t$ & $p$ \\
\hline (Constant) & .777 & 4.199 & .000 & & .727 & 4.298 & .000 \\
Global Awareness & & & & & & & \\
Pretest Score & .128 & 2.216 & .028 & & .136 & 2.412 & .017 \\
Abroad & .023 & .401 & .689 & & .023 & .405 & .686 \\
Global Perspective & & & & & & & \\
$\begin{array}{l}\text { Posttest Score } \\
\text { Global Learning Course }\end{array}$ & .436 & 7.392 & .000 & & .433 & 7.372 & .000 \\
Enrollment & & & & & & & \\
\hline
\end{tabular}

Note. The DV in this analysis was students' global awareness posttest scores. See Chapter 3 for a description and coding of other variables.

Hypothesis $\mathbf{3}_{\mathrm{e}}$. This hypothesis stated that there would be a significant difference on global awareness posttest scores between those students who were enrolled in a global 
learning course and those who were not when controlling for global awareness pretest scores and previous global learning course completion, independent of global perspective posttest scores. A multiple regression analysis was conducted to test this hypothesis using the following models:

- Full model: y (Global Awareness Posttest Score $)=\mathrm{a}_{0} \mathrm{u}+\mathrm{a}_{37}$ (Global Learning Course Enrollment $)+\mathrm{a}_{38}($ Global Awareness Pretest Score $)+\mathrm{a}_{39}$ (Previous Global Learning Course $)+\mathrm{a}_{40}($ Global Perspective Posttest Score $)+\mathrm{E}_{9}$

- Restricted model: y (Global Awareness Posttest Score) $=\mathrm{a}_{0} \mathrm{u}+\mathrm{a}_{41}($ Global Awareness Pretest Score $)+a_{42}($ Previous Global Learning Course $)+a_{43}($ Global Perspective Posttest Score) $+\mathrm{E}_{10}$ A summary of results of this analysis is presented in Table 15. These results indicated that the global learning course did not account for a significant amount of the unique variance in predicting global awareness posttest scores, independent of the global awareness pretest score, previous global learning course completion, and the global perspective posttest score $(p=.520, \alpha<.05)$. These results did not confirm research hypothesis 3 . The coefficients and significance of each of the models' variables are presented in Table 16.

Table 15

Hypothesis $3_{e}$ : Summary of Results of Regression Analysis

\begin{tabular}{lccccc}
\hline \multicolumn{1}{c}{ Model } & $R^{2}$ & $d f$ & $F$ Change & $p$ & Significance \\
\hline Full & .236 & 4 & .415 & .520 & NS \\
Restricted & .234 & 3 & & & \\
\hline
\end{tabular}


Table 16

Hypothesis $3{ }_{e}$ : Predictor Variables to Determine Global Awareness Posttest Scores

\begin{tabular}{lccccccc}
\hline & \multicolumn{3}{c}{ Full Model } & & \multicolumn{3}{c}{ Restricted Model } \\
\cline { 2 - 4 } \cline { 5 - 7 } Variables & $b$ & $t$ & $p$ & & $b$ & $t$ & $p$ \\
\hline (Constant) & .840 & 6.148 & .000 & & .794 & 6.833 & .000 \\
$\begin{array}{l}\text { Global Awareness } \\
\text { Pretest Score }\end{array}$ & .128 & 2.225 & .027 & & .136 & 2.419 & .016 \\
$\begin{array}{l}\text { Previous Global } \\
\text { Learning Course }\end{array}$ & -.040 & -.377 & .707 & & -.046 & -.432 & .666 \\
$\begin{array}{l}\text { Global Perspective } \\
\text { Posttest Score }\end{array}$ & .433 & 7.224 & .000 & & .430 & 7.205 & .000 \\
$\begin{array}{l}\text { Global Learning } \\
\text { Course Enrollment }\end{array}$ & -.063 & -.644 & .520 & & & & \\
\hline
\end{tabular}

Note. The DV in this analysis was students' global awareness posttest scores. See Chapter 3 for a description and coding of other variables.

Hypothesis $\mathbf{3}_{\mathrm{f}}$. This hypothesis stated that there would be a significant difference on global awareness posttest scores between students who earned a "B" and above in a global learning course and students who earned a " $D$ " and below in a global learning course. A simple regression analysis was conducted to test this hypothesis using the following model:

- Full model: y (Global Awareness Posttest Score $)=\mathrm{a}_{0} \mathrm{u}+\mathrm{a}_{44}($ Global Learning Grade) $+E_{11}$

A summary of results of this analysis is presented in Table 17. These results indicated that the global learning course grade did not account for a significant amount of the unique variance in predicting global awareness posttest scores $(p=.567, \alpha<.05)$. These results did not confirm research hypothesis $3_{\mathrm{f}}$. The coefficients and significance of each of the models' variables are presented in Table 18. 
Table 17

Hypothesis $3_{f}$ : Summary of Results of Regression Analysis

\begin{tabular}{rccccc}
\hline Model & $R^{2}$ & $d f$ & $F$ Change & $p$ & Significance \\
\hline Full & .003 & 1 & .330 & .567 & NS \\
\hline
\end{tabular}

Table 18

Hypothesis 3f: Predictor Variable to Determine Global Awareness Posttest Scores

\begin{tabular}{|c|c|c|c|}
\hline \multirow[b]{2}{*}{ Variables } & \multicolumn{3}{|c|}{ Full Model } \\
\hline & $b$ & $t$ & $p$ \\
\hline (Constant) & 1.259 & 6.633 & .000 \\
\hline $\begin{array}{l}\text { Global Learning } \\
\text { Grade }\end{array}$ & .119 & .574 & .567 \\
\hline
\end{tabular}

Note. The DV in this analysis was students' global awareness posttest scores. See Chapter 3 for a description and coding of other variables.

Post hoc Analysis. Global awareness pretest scores were used to control for initial differences between treatment and control groups. A post hoc independent-samples $t$-test found that there was a significant difference $(\alpha<.05)$ in mean global awareness pretest scores between students enrolled in a global learning course $(M=1.51, S D=.75)$ and students enrolled in a non-global learning course $(M=1.85, S D=.91) ; t(218)=$ $3.015, p=.003$. Since the difference between group pretest scores was associated with the treatment in the ANCOVA models and a non-linear relationship between these variables might produce a differential effect on the DV, interaction was tested. One of the assumptions of ANCOVA is that there is a linear relationship among predictor variables in the model; in other words, one predictor's effect on the DV does not influence the effect of another predictor on the DV. Dependent relationships between or among predictors are known as interactions (McNeil, et al., 2012). Interaction among predictor 
variables may increase the probability of committing a Type IV error, "the incorrect interpretation of a correctly rejected hypothesis" (Marascuilo \& Levin, 1970, p. 398).

The post hoc regression analysis revealed a significant interaction between student global awareness pretest scores and the treatment in predicting global awareness posttest scores, $F(3,216)=4.354, p=.005$. Figure 1 shows the non-linear relationship between the two predictor variables, global awareness pretest scores and treatment. The figure displays the differential effect of treatment on students' global awareness posttest scores at varying levels of their global awareness pretest scores.

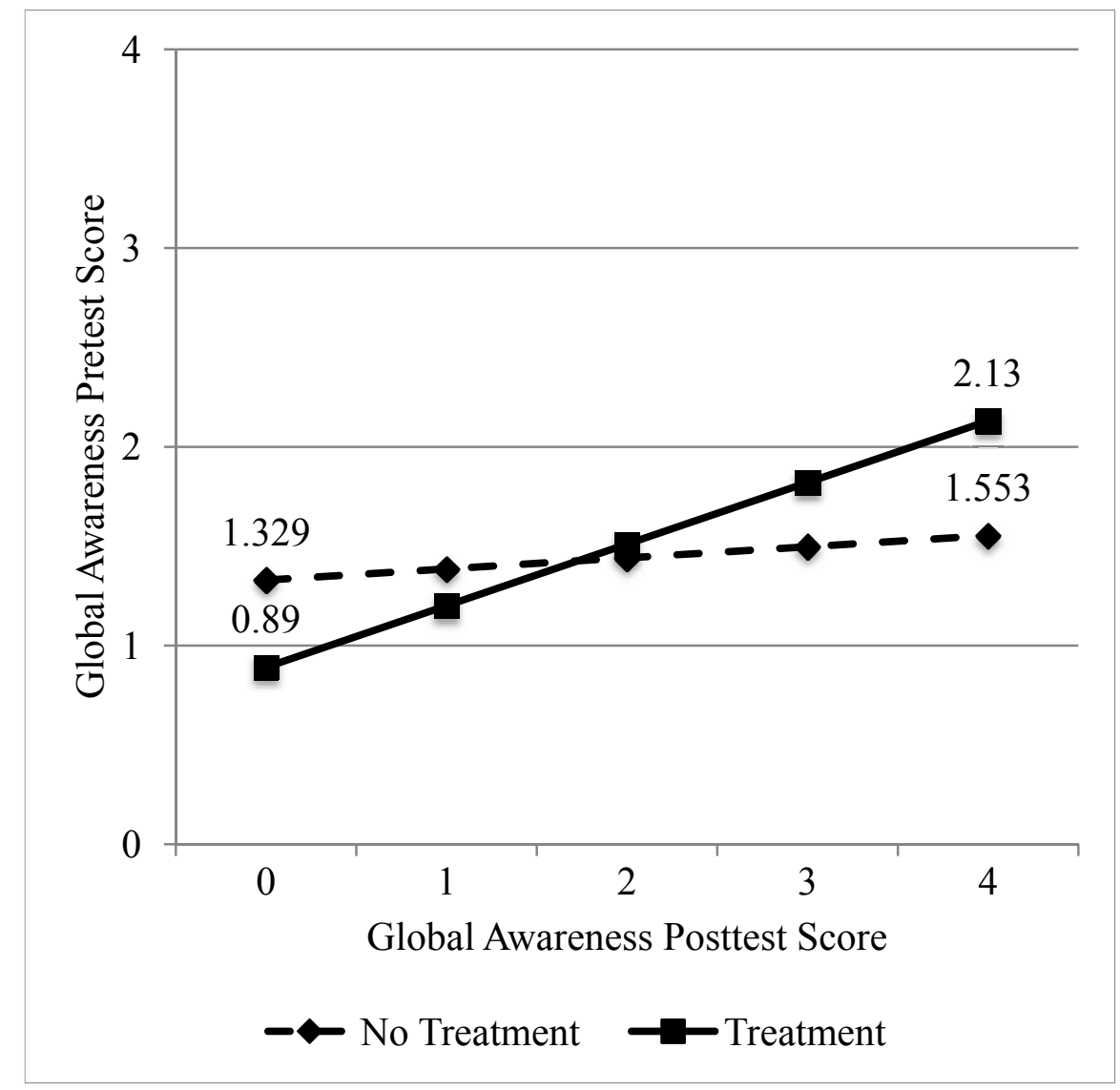

Figure 1. Interaction between global awareness pretest score and treatment 


\section{Research Question 4}

To what extent does evidence support the validity of scores yielded from a rubric measuring students' global perspective?

Hypothesis $4_{a}$. This hypothesis stated that there would be a significant difference on global perspective posttest scores between those students who were enrolled in a global learning course and those who were not when controlling for global perspective pretest scores and class status, independent of global awareness posttest scores. A multiple regression analysis was conducted to test this hypothesis using the following models:

- Full model: y (Global Perspective Posttest Score) $=\mathrm{a}_{0} \mathrm{u}+\mathrm{a}_{45}$ (Global Learning Course Enrollment $)+\mathrm{a}_{46}($ Global Perspective Pretest Score $)+\mathrm{a}_{47}($ Status $)+$ $\mathrm{a}_{48}($ Global Awareness Posttest Score $)+\mathrm{E}_{12}$

- $\quad$ Restricted model: y (Global Perspective Posttest Score $)=\mathrm{a}_{\mathrm{o}} \mathrm{u}+\mathrm{a}_{49}$ (Global Perspective Pretest Score $)+\mathrm{a}_{50}($ Status $)+\mathrm{a}_{51}($ Global Awareness Posttest Score $)+$ $\mathrm{E}_{13}$

A summary of results of this analysis is presented in Table 19. These results indicated that the global learning course did not account for a significant amount of the unique variance in predicting global perspective posttest scores, independent of the global perspective pretest score, class status, and the global awareness posttest score $(p=.185$, $\alpha<.05)$. These results did not confirm research hypothesis $4_{a}$. The coefficients and significance of each of the models' variables are presented in Table 20. 
Table 19

Hypothesis $4_{a}$ : Summary of Results of Regression Analysis

\begin{tabular}{lccccc}
\hline \multicolumn{1}{c}{ Model } & $R^{2}$ & $d f$ & $F$ Change & $p$ & Significance \\
\hline Full & .239 & 4 & 1.770 & .185 & NS \\
Restricted & .233 & 3 & & & \\
\hline
\end{tabular}

Table 20

Hypothesis $4_{a}$ : Predictor Variables to Determine Global Perspective Posttest Scores

\begin{tabular}{|c|c|c|c|c|c|c|}
\hline \multirow[b]{2}{*}{ Variables } & \multicolumn{3}{|c|}{ Full Model } & \multicolumn{3}{|c|}{ Restricted Model } \\
\hline & $b$ & $t$ & $p$ & $b$ & $t$ & $p$ \\
\hline (Constant) & .098 & .561 & .575 & .233 & 1.637 & .103 \\
\hline Global Perspective & & & & & & \\
\hline Pretest Score & .145 & 2.203 & .029 & .126 & 1.963 & .051 \\
\hline Status & -.042 & -.806 & .421 & -.061 & -1.203 & .230 \\
\hline $\begin{array}{l}\text { Global Awareness } \\
\text { Posttest Score }\end{array}$ & .456 & 7.418 & .000 & .455 & 7.380 & .000 \\
\hline $\begin{array}{l}\text { Global Learning } \\
\text { Course Enrollment }\end{array}$ & .137 & 1.330 & .185 & & & \\
\hline
\end{tabular}

Note. The DV in this analysis was students' global perspective posttest scores. See Chapter 3 for a description and coding of other variables.

Hypothesis $\mathbf{4}_{b}$. This hypothesis stated that there would be a significant difference on global perspective posttest scores between those students who were enrolled in a global learning course and those who were not, when controlling for global perspective pretest scores and race/ethnicity, independent of global awareness posttest scores. A multiple regression analysis was conducted to test this hypothesis using the following models: 
- Full model: y (Global Perspective Posttest Score) $=\mathrm{a}_{0} \mathrm{u}+\mathrm{a}_{52}$ (Global Learning Course Enrollment $)+\mathrm{a}_{53}($ Global Perspective Pretest Score $)+\mathrm{a}_{54}($ EurWhite $)+$ $\mathrm{a}_{55}($ AsnPacI $)+\mathrm{a}_{56}($ HispLat $)+\mathrm{a}_{57}$ (Mult $)+\mathrm{a}_{58}($ UnkOther $)+\mathrm{a}_{59}$ (Global Awareness Posttest Score) $+\mathrm{E}_{14}$

- Restricted model: $\mathrm{y}$ (Global Perspective Posttest Score) $=\mathrm{a}_{0} \mathrm{u}+\mathrm{a}_{60}($ Global Perspective Pretest Score $)+\mathrm{a}_{61}($ EurWhite $)+\mathrm{a}_{62}($ AsnPacI $)+\mathrm{a}_{63}($ HispLat $)+$ $\mathrm{a}_{64}($ Mult $)+\mathrm{a}_{65}($ UnkOther $)+\mathrm{a}_{66}($ Global Awareness Posttest Score $)+\mathrm{E}_{15}$ A summary of results of this analysis is presented in Table 21 . These results indicated that the global learning course did not account for a significant amount of the unique variance in predicting global perspective posttest scores, independent of the global perspective pretest score, race/ethnicity, and the global awareness posttest score ( $p=$ $.111, \alpha<.05)$. These results did not confirm research hypothesis $4_{\mathbf{b}}$. The coefficients and significance of each of the models' variables are presented in Table 22.

Table 21

Hypothesis $4_{\boldsymbol{b}}$ : Summary of Results of Regression Analysis

\begin{tabular}{lccccc}
\hline \multicolumn{1}{c}{ Model } & $R^{2}$ & $d f$ & $F$ Change & $p$ & Significance \\
\hline Full & .246 & 8 & 2.557 & .111 & NS \\
Restricted & .237 & 7 & & & \\
\hline
\end{tabular}


Table 22

Hypothesis $4_{\boldsymbol{b}}$ : Predictor Variables to Determine Global Perspective Posttest Scores

\begin{tabular}{|c|c|c|c|c|c|c|}
\hline \multirow[b]{2}{*}{ Variables } & \multicolumn{3}{|c|}{ Full Model } & \multicolumn{3}{|c|}{ Restricted Model } \\
\hline & $b$ & $t$ & $p$ & $b$ & $t$ & $p$ \\
\hline (Constant) & .010 & .067 & .946 & .141 & 1.155 & .249 \\
\hline Global Perspective & & & & & & \\
\hline Pretest Score & .148 & 2.217 & .028 & .127 & 1.930 & .055 \\
\hline European/White & .126 & .888 & .376 & .106 & .743 & .458 \\
\hline African American & -.059 & -.415 & .678 & -.081 & -.568 & .571 \\
\hline $\begin{array}{l}\text { Asian/Pacific } \\
\text { Islander }\end{array}$ & -.218 & -.961 & .338 & -.213 & -.935 & .351 \\
\hline Multiple Ethnicities & .090 & .472 & .637 & .102 & .534 & .594 \\
\hline Unknown/Other & -.013 & -.051 & .960 & -.066 & -.266 & .791 \\
\hline $\begin{array}{l}\text { Global Awareness } \\
\text { Posttest Score }\end{array}$ & .450 & 7.167 & .000 & .447 & 7.087 & .000 \\
\hline $\begin{array}{l}\text { Global Learning } \\
\text { Course Enrollment }\end{array}$ & .162 & 1.599 & .111 & & & \\
\hline
\end{tabular}

Note. No participants reported American Indian/Alaskan Native race/ethnicity, therefore this variable was excluded from analysis. The variable Hispanic/Latino was excluded as a constant. The DV in this analysis was students' global perspective posttest scores. See Chapter 3 for a description and coding of other variables.

Hypothesis $4_{c}$. This hypothesis stated that there would be a significant difference on global perspective posttest scores between those students who were enrolled in a global learning course and those who were not when controlling for global perspective pretest scores and fluency in more than one language, independent of global awareness posttest scores. A multiple regression analysis was conducted to test this hypothesis using the following models: 
- Full model: y (Global Perspective Posttest Score $)=\mathrm{a}_{0} \mathrm{u}+\mathrm{a}_{68}$ (Global Learning Course Enrollment $)+\mathrm{a}_{69}($ Global Perspective Pretest Score $)+\mathrm{a}_{70}($ Language $)+$ $\mathrm{a}_{71}($ Global Awareness Posttest Score $)+\mathrm{E}_{16}$

- Restricted model: $\mathrm{y}$ (Global Perspective Posttest Score $)=\mathrm{a}_{0} \mathrm{u}+\mathrm{a}_{72}($ Global Perspective Pretest Score $)+a_{73}($ Language $)+a_{74}($ Global Awareness Posttest Score) $+E_{17}$

A summary of results of this analysis is presented in Table 23. The coefficients and significance of each of the models' variables are presented in Table 24 .

Table 23

Hypothesis $4_{c}$ : Summary of Results of Regression Analysis

\begin{tabular}{lccccc}
\hline \multicolumn{1}{c}{ Model } & $R^{2}$ & $d f$ & $F$ Change & $p$ & Significance \\
\hline Full & .242 & 4 & 2.554 & .112 & NS \\
Restricted & .233 & 3 & & & \\
\hline
\end{tabular}

Table 24

Hypothesis 4c: Predictor Variables to Determine Global Perspective Posttest Scores

\begin{tabular}{|c|c|c|c|c|c|c|}
\hline \multirow[b]{2}{*}{ Variables } & \multicolumn{3}{|c|}{ Full Model } & \multicolumn{3}{|c|}{ Restricted Model } \\
\hline & $b$ & $t$ & $p$ & $b$ & $t$ & $p$ \\
\hline (Constant) & .194 & .965 & .336 & .314 & 1.674 & .095 \\
\hline $\begin{array}{l}\text { Global Perspective } \\
\text { Pretest Score }\end{array}$ & .145 & 2.223 & .027 & .125 & 1.943 & .053 \\
\hline Language & -.102 & -1.239 & .217 & -.102 & -1.245 & .214 \\
\hline $\begin{array}{l}\text { Global Awareness } \\
\text { Posttest Score }\end{array}$ & .457 & 7.453 & .000 & 456 & 7.398 & .000 \\
\hline $\begin{array}{l}\text { Global Learning } \\
\text { Course Enrollment }\end{array}$ & .158 & 1.598 & .112 & & & \\
\hline
\end{tabular}

Note. The DV in this analysis was students' global perspective posttest scores. See Chapter 3 for a description and coding of other variables. 
These results indicated that the global learning course did not account for a significant amount of the unique variance in predicting global perspective posttest scores, independent of the global perspective pretest score, fluency in more than one language, and the global awareness posttest score $(p=.112, \alpha<.05)$. These results did not confirm research hypothesis $4_{c}$.

Hypothesis $\mathbf{4}_{\mathrm{d}}$. This hypothesis stated that there would be a significant difference on global perspective posttest scores between those students who were enrolled in a global learning course and those who were not when controlling for global perspective pretest scores and time spent abroad, independent of global awareness posttest scores. A multiple regression analysis was conducted to test this hypothesis using the following models:

- Full model: $y$ (Global Perspective Posttest Score $)=a_{0} u+a_{75}$ (Global Learning Course Enrollment $)+a_{76}($ Global Perspective Pretest Score $)+a_{77}($ Abroad $)+$ $\mathrm{a}_{78}($ Global Awareness Posttest Score $)+\mathrm{E}_{18}$

- Restricted model: y (Global Perspective Posttest Score) $=\mathrm{a}_{0} \mathrm{u}+\mathrm{a}_{79}($ Global Perspective Pretest Score $)+\mathrm{a}_{80}($ Abroad $)+\mathrm{a}_{81}($ Global Awareness Posttest Score $)$ $+\mathrm{E}_{19}$ A summary of results of this analysis is presented in Table 25. The coefficients and significance of each of the models' variables are presented in Table 26. 
Table 25

Hypothesis $4_{d}$ : Summary of Results of Regression Analysis

\begin{tabular}{lccccc}
\hline \multicolumn{1}{c}{ Model } & $R^{2}$ & $d f$ & $F$ Change & $p$ & Significance \\
\hline Full & .237 & 4 & 2.545 & .112 & NS \\
Restricted & .228 & 3 & & & \\
\hline
\end{tabular}

Table 26

Hypothesis $4_{d}$ : Predictor Variables to Determine Global Perspective Posttest Score

\begin{tabular}{|c|c|c|c|c|c|c|}
\hline \multirow[b]{2}{*}{ Variables } & \multicolumn{3}{|c|}{ Full Model } & \multicolumn{3}{|c|}{ Restricted Model } \\
\hline & $b$ & $t$ & $p$ & $b$ & $t$ & $p$ \\
\hline (Constant) & -.036 & -.200 & .841 & .079 & .470 & .639 \\
\hline Global Perspective & & & & & & \\
\hline Pretest Score & .148 & 2.257 & .025 & .128 & 1.977 & .049 \\
\hline Abroad & .021 & .352 & .726 & .022 & .378 & .706 \\
\hline Global Awareness & & & & & & \\
\hline Posttest Score & .456 & 7.398 & .000 & .454 & 7.343 & .000 \\
\hline Global Learning & & & & & & \\
\hline Course Enrollment & .158 & 1.595 & .112 & & & \\
\hline
\end{tabular}

Note. The DV in this analysis was students' global perspective posttest scores. See Chapter 3 for a description and coding of other variables.

These results indicated that the global learning course did not account for a significant amount of the unique variance in predicting global perspective posttest scores, independent of the global perspective pretest score, time spent abroad, and the global awareness posttest score $(p=.112, \alpha<.05)$. These results did not confirm research hypothesis $4_{d}$.

Hypothesis $4_{\mathrm{e}}$. This hypothesis stated that there would be a significant difference on global perspective posttest scores between those students who were enrolled in a global learning course and those who were not when controlling for global perspective 
pretest scores and previous global learning course completion, independent of global awareness posttest scores. A multiple regression analysis was conducted to test this hypothesis using the following models:

- Full model: y (Global Perspective Posttest Score $)=\mathrm{a}_{0} \mathrm{u}+\mathrm{a}_{82}$ (Global Learning Course Enrollment $)+\mathrm{a}_{83}($ Global Perspective Pretest Score $)+\mathrm{a}_{84}($ Previous Global Learning Course $)+\mathrm{a}_{85}($ Global Awareness Posttest Score $)+\mathrm{E}_{20}$

- Restricted model: $y($ Global Perspective Posttest Score $)=\mathrm{a}_{0} \mathrm{u}+\mathrm{a}_{86}($ Global Perspective Pretest Score $)+a_{87}($ Previous Global Learning Course $)+a_{88}($ Global Awareness Posttest Score) $+E_{21}$ A summary of results of this analysis is presented in Table 27.

Table 27

Hypothesis $4_{e}$ : Summary of Results of Regression Analysis

\begin{tabular}{lccccc}
\hline \multicolumn{1}{c}{ Model } & $R^{2}$ & $d f$ & $F$ Change & $p$ & Significance \\
\hline Full & .257 & 4 & 3.192 & .075 & $\mathrm{~S}$ \\
Restricted & .246 & 3 & & & \\
\hline
\end{tabular}

Using a one-tailed test of significance, these results indicated that the global learning course did account for a significant amount of the unique variance in predicting global perspective posttest scores, independent of the global perspective pretest score, previous enrollment in a global learning course, and the global awareness posttest score $(p=.0375, \alpha<.05)$. These results confirmed research hypothesis $4_{\mathbf{e}}$. The coefficients and significance of each of the models' variables are presented in Table 28. 
Table 28

Hypothesis $4_{e}$ : Predictor Variables to Determine Global Perspective Posttest Scores

\begin{tabular}{|c|c|c|c|c|c|c|}
\hline \multirow[b]{2}{*}{ Variables } & \multicolumn{3}{|c|}{ Full Model } & \multicolumn{3}{|c|}{ Restricted Model } \\
\hline & $b$ & $t$ & $p$ & $b$ & $t$ & $p$ \\
\hline (Constant) & .080 & .592 & .555 & .207 & 1.791 & .075 \\
\hline $\begin{array}{l}\text { Global Perspective } \\
\text { Pretest Score }\end{array}$ & .159 & 2.457 & .015 & .136 & 2.137 & .034 \\
\hline $\begin{array}{l}\text { Previous Global } \\
\text { Learning Course }\end{array}$ & -.259 & -2.436 & .016 & -.246 & -2.307 & .022 \\
\hline $\begin{array}{l}\text { Global Awareness } \\
\text { Posttest Score }\end{array}$ & .441 & 7.215 & .000 & .440 & 7.159 & .000 \\
\hline $\begin{array}{l}\text { Global Learning } \\
\text { Course Enrollment }\end{array}$ & .175 & 1.787 & .075 & & & \\
\hline
\end{tabular}

Note. The DV in this analysis was students' global perspective posttest scores. See Chapter 3 for a description and coding of other variables.

Hypothesis $4_{\mathbf{f}}$. This hypothesis stated that there would be a significant difference on global perspective rubric scores between students who earned a "B" and above in a global learning course and students who earned a " $D$ " and below in a global learning course. A simple regression analysis was conducted to test this hypothesis using the following model:

- Full model: y (Global Perspective Posttest Score) $=\mathrm{a}_{\mathrm{o}} \mathrm{u}+\mathrm{a}_{89}$ (Global Learning Grade) $+\mathrm{E}_{22}$

A summary of results of this analysis is presented in Table 29. These results indicated that the global learning course grade did not account for a significant amount of the unique variance in predicting global awareness posttest scores $(p=.941, \alpha<.05)$. These results did not confirm research hypothesis $4_{\mathbf{f}}$. The coefficients and significance of each of the models' variables are presented in Table 30. 
Table 29

Hypothesis 4f: Summary of Results of Regression Analysis

\begin{tabular}{rccccc}
\hline Model & $R^{2}$ & $d f$ & $F$ Change & $p$ & Significance \\
\hline Full & .000 & 1 & .005 & .941 & NS \\
\hline
\end{tabular}

Table 30

Hypothesis $4_{f}$ : Predictor Variable to Determine Global Perspective Posttest Scores

\begin{tabular}{lccl}
\hline & \multicolumn{3}{c}{ Full Model } \\
\cline { 2 - 4 } Variables & $b$ & $t$ & $p$ \\
\hline (Constant) & .944 & 4.589 & .000 \\
Global Learning & & \\
Grade & -.017 & -.074 & .941 \\
\hline Note. The DV in this analysis was students' global perspective posttest scores. See
\end{tabular}

Note. The DV in this analysis was students' global perspective posttest scores. See Chapter 3 for a description and coding of other variables.

Post hoc Analysis. Along with global awareness pretest scores, global perspective pretest scores were used to control for initial differences between treatment and control groups. A post hoc independent-samples $t$-test found that there was a significant difference $(\alpha<.05)$ in mean global perspective pretest scores between students in a global learning course $(M=.90, S D=.66)$ and students in a non-global learning course $(M=1.2, S D=.84) ; t(218)=2.987, p=.003$. Since this difference was associated with the treatment in the ANCOVA models, interaction was tested.

The post hoc regression analysis revealed a significant interaction between student global perspective pretest scores and the treatment in predicting global perspective posttest scores, $F(3,216)=4.464, p=.005$. Figure 2 shows the non-linear relationship between the two predictor variables, global perspective pretest scores and treatment. The figure displays the differential effect of treatment on students' global perspective posttest scores at varying levels of their global awareness pretest scores. 


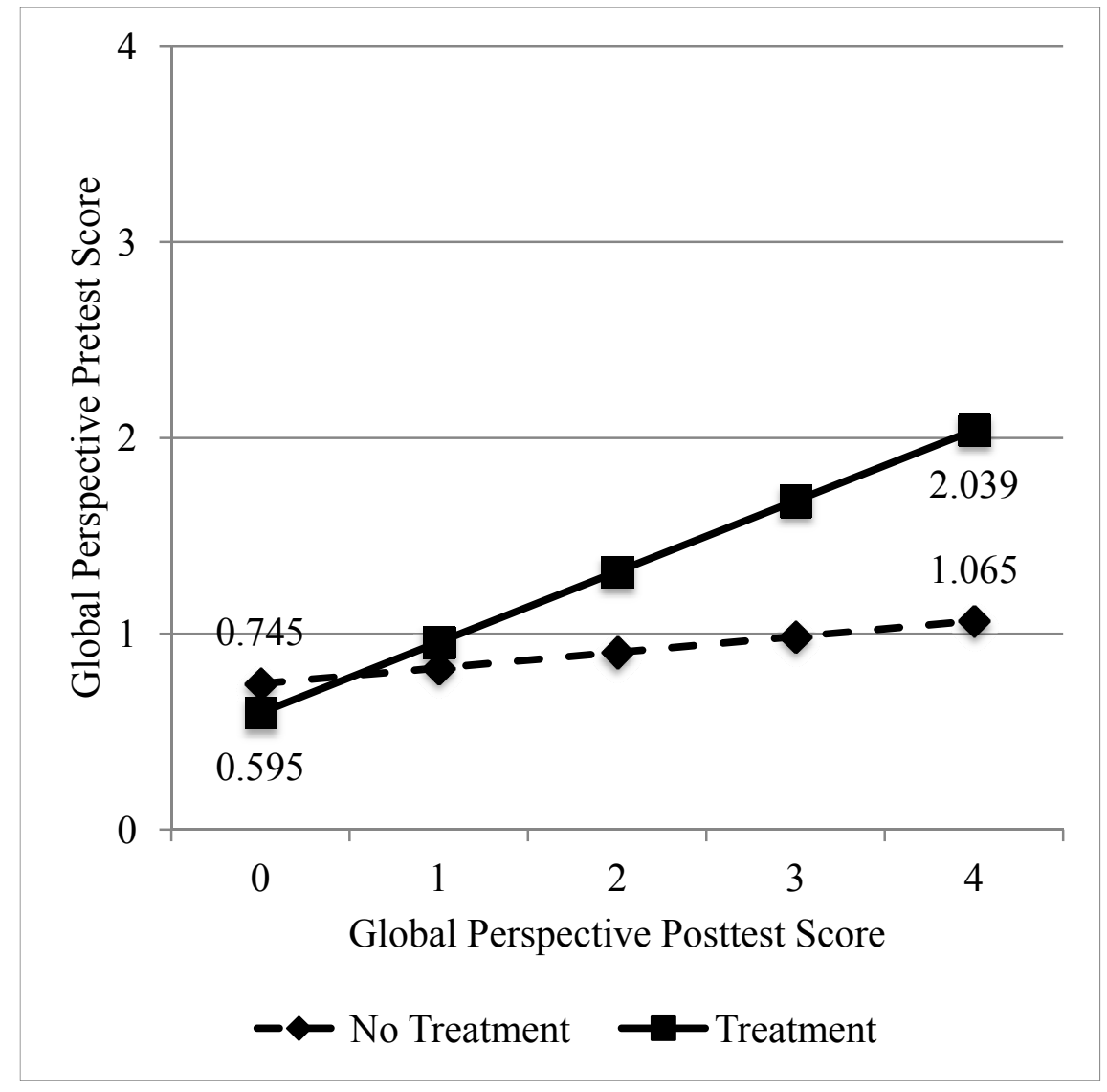

Figure 2. Interaction between global perspective pretest score and treatment

\section{Summary}

This chapter presented an explanation of how the final sample was determined, a description and crosstabulation analysis of the study's participants, and the results of statistical tests for each of the study's research hypotheses.

The study's final matched-pair sample was composed of a total of 220 students, with 132 students (60\%) enrolled in a global learning course and 88 students (40\%) enrolled in a non-global learning course. Statistical analysis was conducted to explore the equivalence of the treatment and control groups. Crosstabulation analysis found that the groups were similar in terms of race/ethnicity, number of languages spoken fluently, time spent abroad, and previous completion of a global learning course. The groups differed 
significantly, however, in terms of students' class status. The treatment group was composed of $69.7 \%$ freshmen, $14.4 \%$ sophomores, $9.8 \%$ juniors, and $6.1 \%$ seniors, as opposed to $34.1 \%$ freshmen, $39.8 \%$ sophomores, $17 \%$ juniors, and $9.1 \%$ seniors in the control group. There was also a significant difference in mean global awareness and mean global perspective pretest scores between the groups in the final sample.

The research hypotheses served as the basis for presentation of results of the data analyses. Research hypotheses 1 and 2 addressed the percentage of inter-rater agreement as evidence of the extent to which the rubrics were reliable. Among raters using the global awareness rubric for the pretest, agreement exceeded the minimum .80 reliability requirement (.89) and a chi-square test of frequency found these results statistically significant $(p<.0001)$. Posttest rater agreement also exceeded the minimum requirement (.95) and was found statistically significant $(p<.0001)$. Agreement also exceeded the reliability requirement among raters using the global perspective rubric to score the pretest $(.92, p<.0001)$ and posttest $(.91, p<.0001)$. These results confirmed both research hypotheses 1 and 2.

Research hypotheses $3_{\mathrm{a}}$ through $3 \mathrm{f}$ addressed the validity of the global awareness rubric. Hypotheses $3_{\mathrm{a}}$ through $3_{\mathrm{e}}$ stated that there would be a significant difference on global awareness posttest scores between those students enrolled in a global learning course and those who were not when controlling for global awareness pretest scores and an extraneous variable, independent of global perspective posttest scores. Multiple regression analyses were used to test these hypotheses, wherein the full regression model was tested against the restricted model in order to determine if the primary IV, the global learning course, accounted for a significant amount of the unique variance in predicting 
the DV, global awareness posttest scores, independent of the covariates. Using one-tailed tests of significance at the .05 level, these regression analyses found no significant differences between treatment and control groups on global awareness posttest scores, independent of global awareness pretest scores and global perspective posttest scores, when controlling for the extraneous variables: (a) class status; (b) race/ethnicity; (c) fluency in more than one language; (d) time spent abroad; and (e) previous global learning course completion. A simple regression analysis was used to test hypothesis $3_{\mathrm{f}}$, which stated that there would be a significant difference on global awareness posttest scores between students who earned a "B" and above in a global learning course and students who earned a "D" and below in a global learning course. No significant difference between the comparison groups was found. On the basis of these results, hypotheses $3_{\mathrm{a}}$ through $3_{\mathrm{f}}$ were not confirmed.

A post hoc independent-samples $t$-test found that there was a significant difference $(\alpha<.05)$ in mean global awareness pretest scores between students enrolled in a global learning course $(M=1.51, S D=.75)$ and students enrolled in a non-global learning course $(M=1.85, S D=.91) ; t(218)=3.015, p=.003$. A post hoc regression analysis revealed a significant interaction between student global awareness pretest scores and the treatment in predicting global awareness posttest scores, $F(3,216)=$ $4.354, p=.005$.

The validity of the global perspective rubric was explored through research hypotheses $4_{\mathrm{a}}$ through $4_{\mathrm{f}}$. Hypotheses $4_{\mathrm{a}}$ through $4_{\mathrm{e}}$ stated that there would be a significant difference on global perspective posttest scores between those students enrolled in a global learning course and those who were not when controlling for global 
perspective pretest scores and an extraneous variable, independent of global awareness posttest scores. Multiple regression analyses were used to test these hypotheses, wherein the full regression model was tested against the restricted model in order to determine if the primary IV, the global learning course, accounted for a significant amount of the unique variance in predicting the DV, global perspective posttest scores, independent of the covariates. For hypotheses $4_{a}$ through $4_{d}$, these analyses found no significant differences at the .05 level between treatment and control groups on global perspective posttest scores, independent of global perspective pretest scores and global awareness posttest scores, when controlling for the extraneous variables: (a) class status; (b) race/ethnicity; (c) fluency in more than one language; and (d) time spent abroad. On the basis of these results, hypotheses $4_{a}$ through $4_{d}$ were not supported. Using a one-tailed test of significance, regression analysis for hypothesis $4_{\mathbf{e}}$ did find a significant difference on global perspective posttest scores between those students who were enrolled in a global learning course and those who were not when controlling for global perspective pretest scores and previous global learning course completion, independent of global awareness posttest scores $(p=.03755, \alpha<.05)$. These results confirmed hypothesis $4_{\mathrm{e}}$. A simple regression analysis conducted to test hypothesis $4_{\mathrm{f}}$ did not reveal a significant difference in global perspective posttest scores between students who earned a "B" and above in a global learning course and students who earned a " $\mathrm{D}$ " and below in a global learning course. On the basis of these results, hypothesis $4_{f}$ was not confirmed.

A post hoc independent-samples $t$-test found a significant difference $(\alpha<.05)$ in mean global perspective pretest scores between students enrolled in a global learning course $(M=.90, S D=.66)$ and students enrolled in a non-global learning course 
$(M=1.2, S D=.84) ; t(218)=2.987, p=.003$. A post hoc test also revealed a significant interaction between global perspective pretest scores and the treatment in predicting global perspective posttest scores, $F(3,216)=4.464, p=.005$. 


\section{CHAPTER V}

\section{DISCUSSION}

This chapter begins with a summary of the study, including a presentation of answers to each of the study's research questions based on test results for the study's research hypotheses. The chapter continues with an interpretation and analysis of the results as they relate to the relevant literature. The chapter concludes with study limitations, implications for practice, and recommendations for future research.

\section{Summary of the Study}

Today's young adults are citizens in a diverse and interconnected world. The issues and problems they face - whether national, international, or global in scope - are complex, ill-structured, and shaped by shifting dynamics. In order to think critically and make responsible decisions concerning these challenges, undergraduates must understand how local, global, international, and intercultural issues, trends, and systems are interrelated, and they must be able to analyze problems from multiple perspectives (Adams \& Carfagna, 2006; American Council on International Intercultural Education Conference, 1996; Hunter, White, \& Godbey, 2006). Knowledge of interrelationships among issues, trends, and systems across the globe has been called global awareness (Lemke, 2002). The ability to examine the world via diverse cultural, intellectual, and spiritual points of view has been called global perspective (Braskamp, et al., 2009). Increasingly, students view themselves as citizens of not only local and national communities, but also of the global community (Education Development Center, 2006; Our World Alliance, 2006). To address this complex, multi-layered conception of affiliation, colleges and universities across the United States have implemented global 
learning initiatives to prepare students for global citizenship; "the willingness of individuals to apply their knowledge of interrelated issues, trends, and systems and multiperspective analytical skills to local, global, international, and intercultural problem solving" (Florida International University, 2010, p. 58). Global learning is the process by which students are prepared to fulfill their civic responsibilities in a diverse and interconnected world (Hovland, 2006). Global learning is also a term that has been used to describe specific content, pedagogy, and assessment strategies that enable students to develop SLOs associated with global citizenship, that is global awareness and global perspective (Florida International University, 2010).

Global awareness and global perspective were two SLOs for an institution-wide global learning initiative at a large, Hispanic-serving, urban, public, research university in South Florida. The purpose of this initiative was to provide all undergraduate students with curricular and co-curricular opportunities to develop these outcomes through global learning. All undergraduates, both native and transfer, were required to take a minimum of two global learning courses - one as part of the general education curriculum and one as part of their major program of study — and participate in co-curricular activities designed to increase their global awareness and global perspective. Global learning courses were developed to enhance these outcomes through components such as international and global content, active learning strategies, team teaching, integrated cocurricular activities, and interdisciplinary and problem-based curricula.

Despite the marked increase in global learning initiatives in recent years, global learning assessment options remain limited. A review of research for the initiative and this study yielded a lack of instruments designed to measure global awareness and global 
perspective in the context of an authentic performance assessment. The purpose of this quasi-experimental study was to demonstrate the extent to which evidence supported the validity and reliability of scores yielded from rubrics developed to measure undergraduate students' global awareness and global perspective. The study utilized a pretest/posttest nonequivalent group design to ascertain the rubrics' ability to discern and compare average learning gains of undergraduate students enrolled in two global learning courses and students enrolled in two non-global learning courses. This study addressed the following four research questions:

1. To what extent does evidence support the reliability of scores yielded from a rubric measuring students' global awareness?

2. To what extent does evidence support the reliability of scores yielded from a rubric measuring students' global perspective?

3. To what extent does evidence support the validity of scores yielded from a rubric measuring students' global awareness?

4. To what extent does evidence support the validity of scores yielded from a rubric measuring students' global perspective?

\section{Setting and Participants}

This study was conducted at a large, Hispanic-serving, urban, public, research university in South Florida with approximately 32,901 undergraduate students. It involved a purposive matched-pair sample of 220 students, of whom 132 students $(60 \%)$ were enrolled in a global learning course and 88 students (40\%) were enrolled in a nonglobal learning course. Crosstabulation analysis of participants' demographic characteristics determined that the treatment and control groups were similar in terms of 
race/ethnicity, number of languages spoken fluently, time spent abroad, and previous completion of a global learning course. The groups differed significantly, however, in terms of students' class status. Post hoc independent-samples $t$-tests found that the groups also differed significantly in terms of their global awareness and global perspective pretest scores.

\section{Methods}

Research hypotheses were developed to address each of the study's research questions. This study utilized a pretest/posttest nonequivalent group design to compare the average learning gains of students enrolled in global learning and non-global learning courses. The study's pretest was administered to students during the first two weeks of the semester and the posttest was administered during the last two weeks of the semester. Trained faculty raters used the rubrics to score completed assessments. Hypotheses associated with research questions 1 and 2 concerned the rubrics' reliability. Reliability in this study was operationally defined as the percentage of inter-rater agreement. Interrater reliability of at least .80 agreement among raters was deemed necessary to meet the minimum reliability requirement.

Research questions 3 and 4 concerned the rubrics' validity. Linear regression (Cohen, et al., 2003; McNeil, et al., 2012) was used to test the hypotheses associated with these questions. One-tailed tests of significance at the .05 level were used to test the research hypotheses because there was reason to believe that the treatment group would do better than the control group. The primary IV in this study was completion of a global learning course. The DVs in this study were student posttest scores on a rubric measuring global awareness and student posttest scores on a rubric measuring global perspective. 
The study controlled for several extraneous variables in order to maximize experimental variance: (a) class status; (b) race/ethnicity; (c) fluency in more than one language; (d) time spent abroad; and (e) previous global learning course completion. End-of-course grades for students enrolled in a global learning course were collected in order to perform a within-group discriminant analysis.

\section{Results}

Research Question 1. Evidence supported the finding that the global awareness rubric yielded scores that were highly reliable measures of students' global awareness. This question was addressed by research hypothesis 1 , which stated that there would be significant inter-rater agreement among raters using the rubric to measure students' pretests and posttests in the treatment and control groups. The hypothesis was confirmed because agreement rates on both the pretest (.89) and posttest (.95) far exceeded the minimum .80 reliability requirement, and these results were found highly significant $(p<$ $.0001)$.

Research Question 2. Evidence also supported the finding that the global perspective rubric yielded highly reliable scores. This question was addressed by research hypothesis 2 , which stated that there would be significant inter-rater agreement among raters who used the global perspective rubric to measure students' pretests and posttests. Because agreement rates on both the pretest (.92) and the posttest (.91) far exceeded the minimum requirement and were found were also found highly significant $(p<.0001)$, the hypothesis was confirmed.

Research Question 3. Evidence supported the finding that the global awareness rubric yielded scores that were valid measures of students' development of this learning 
outcome. Research hypotheses $3_{\mathrm{a}}$ through $3_{\mathrm{f}}$ addressed the validity of the global awareness rubric. Multiple regression analyses used to test hypotheses $3_{\mathbf{a}}$ through $3_{\mathrm{e}}$ did not find significant main effects for the treatment when controlling for pretest scores and extraneous variables, therefore the research hypotheses were not confirmed. Hypothesis $3_{\mathrm{f}}$ was also not confirmed because a simple regression analysis did not reveal a significant difference in global awareness posttest scores between students who earned a "B" and above in a global learning course and students who earned a " $\mathrm{D}$ " and below in a global learning course. However, a post hoc regression analysis did reveal a significant interaction between global awareness pretest scores and treatment. On average, for students who scored above 1.728 on the global awareness pretest, the treatment led to significantly and disproportionately higher global awareness posttest scores than were predicted for students who did not receive the treatment. The treatment did not, on average, have a significant effect for students who scored below 1.728 on the global awareness pretest. This cross-over interaction supported the finding that the global awareness rubric could be used to detect learning differences between the treatment and control groups as well as differences within the treatment group. This evidence strongly supported the validity of the scores yielded from the global awareness rubric.

Research Question 4. Evidence also supported the finding that the global perspective rubric yielded scores that were valid measures of students' development of this learning outcome. Research hypotheses $4_{\mathrm{a}}$ through $4_{\mathrm{f}}$ addressed the validity of the global perspective rubric. Multiple regression analyses used to test hypotheses $4_{\mathrm{a}}$ through $4_{d}$ did not find significant main effects for the treatment when controlling for pretest scores and extraneous variables, therefore these research hypotheses were not confirmed. 
Significant main effects were found, however, for hypothesis $4_{e}$, which was confirmed. A simple regression analysis conducted to test hypothesis $4_{\mathrm{f}}$ did not reveal a significant difference in global awareness posttest scores between students who earned a "B" and above in a global learning course and students who earned a "D" and below in a global learning course, therefore this hypothesis was not confirmed. As was found for the global awareness rubric, however, a post hoc regression analysis revealed significant interaction between global perspective pretest scores and treatment. On average, the treatment led to significantly higher global perspective posttest scores for students who scored above .533 on the global perspective pretest than were predicted for students who did not receive the treatment. The treatment did not, on average, have a significant effect for students who scored below .533 on the pretest. This cross-over interaction supported the finding that the global perspective rubric could be used to detect learning differences between the treatment and control groups as well as within the treatment group. This evidence was strong support for the validity of the scores yielded from the global perspective rubric.

\section{Interpretation and Analysis of Results}

This study derived its theoretical foundation from constructivism, which guided its approach to research methods, teaching, learning, and assessment. The study's methodology was based on a unified concept of validity, such that interrelated aspects of construct validity and reliability were explored as sources of evidence to build a case for the appropriateness, meaningfulness, and usefulness of scores yielded from the assessment instrument (Messick, 1996). In terms of teaching and learning, the study was informed by Cognitive Flexibility Theory (CFT), which suggests that learning and cognition in ill-structured content domains (ISDs) require individuals to flexibly apply 
background knowledge and skills to unique cases (Spiro \& DeSchryver, 2009; Spiro, Vispoel, Schmitz, Samarapungavan, \& Boerger, 1987). According to CFT, individuals must be able to consider multiple unanticipated contextual variables and a variety of sometimes unrelated precedents in order to think critically in ISDs. The study was further informed by the work of global education researchers who view global awareness and global perspective as distinct yet highly interrelated outcomes of global learning (Case, 1993; Hanvey, 1975; Merryfield; 2008; Selby \& Pike, 2000). These researchers have asserted that global awareness and global perspective result from a constructivist approach to global learning that includes international and global content, active learning strategies, team teaching, integrated co-curricular activities, and interdisciplinary and problem-based curricula.

\section{Reliability}

The study found high percentages of inter-rater agreement, far exceeding the .80 minimum requirement, among raters using the rubrics to assess students' global awareness and global perspective. These results were expected, as the rubrics' development and rater training procedures were based on best practices described in the research literature. Each of the holistic rubrics contained no more than five scoring scale levels, and descriptive performance criteria were included to assist raters in distinguishing qualitative response differences pertaining to each score level (Popham, 1997). The scoring scale and criteria followed a continuum grounded in an underlying theoretical framework, Bloom's (1956) Taxonomy of Cognitive Development. Attributes described in the performance criteria aligned with the framework and were depicted consistently and progressively from one level to the next (Tierney \& Simon, 2004). The 
language used to explain the criteria was specific and was generated by faculty and raters who observed response trends during pilot studies of the instrument. Additionally, benchmark responses derived from the pilot studies were included in the rubric to operationalize the scoring criteria (Popp, Ryan, \& Thompson, 2009). All of these rubric attributes contributed to the raters' ability to place "the desired emphasis on specific, uniform criteria, so that the role of subjective opinions is minimized" (Newell, Dahm, \& Newell, as cited in Stellmack, et al., 2009, p. 103).

The high rates of agreement were also consistent with the literature on recommended training protocols for raters using rubrics to score open-ended questions. These training protocols were implemented during the data collection phase of the study. Training sessions began with study and discussion of the rubrics, followed by collaborative and individual scoring of anchor papers distributed along levels of the scoring scale. Training concluded with a sample scoring session of $10 \%$ of the total papers to be scored to establish an agreement rate of at least .80 (Gearhart, 1994). To resolve discrepant scores, defined in this study as a difference of more than one point between two trained raters' scores, a third trained rater was asked to score the response and the final score was an average of the three raters' scores. This score resolution method has been found to contribute to higher rates of agreement as compared to alternative methods (Johnson, Penny, \& Gordon, 2000).

The study's findings concerning inter-rater agreement provided empirical support for the rubrics' reliability, but they also pointed to the rubrics' structural integrity, a key source of evidence for construct validity (Messick, 1989; Miller \& Linn, 2000; Popp, Ryan, \& Thompson, 2009). Loevinger (1957) pioneered the concept of structural validity, 
which "refers to the extent to which structural relations between test items parallel the structural relations of other manifestations of the trait being measured" (p. 661). The structural validity of rubrics is rarely discussed in research literature. Evidence of the structural validity of criterion-based tests and surveys is commonly gathered through intercorrelations among items or through factor analyses. However, in her discussion of class models of traits as one type of underlying assessment structure, Loevinger (1957) implied that structural validity is an important characteristic of rubrics. Inter-rater agreement has been specifically cited as a source of evidence for rubrics' structural validity (Gadbury-Amyot, et al., 2003; Miller \& Linn, 2000).

\section{Validity}

Treatment effects between groups. One of the unexpected results of this study concerned the rubrics' ability to discern the differential effect of the global learning course on student achievement of the learning outcomes. An assumption of this study was that global learning courses were comprised of substantively different learning strategies than non-global learning courses and that students in global learning courses would make greater learning gains in global awareness and global perspective than students in nonglobal learning courses. If this assumption held true and the rubrics could be used to detect learning differences between groups, then this would support the rubrics' substantive validity, an important contribution to the overall assessment of the rubrics' construct validity. Messick (1994) asserted that the substantive aspect of construct validity is supported by empirical evidence that the processes sampled by the performance are those in which the student is actually engaged. Miller and Linn (2000) 
specifically recommended pretest/posttest design experiments comparing contexts in which the process model is taught as a source of evidence for substantive validity.

The ANCOVAs conducted to test between group comparisons did not reveal significant main effects for the treatment in predicting either global awareness or global perspective posttest scores. However, one of the assumptions of ANCOVA is that there is a linear relationship among predictor variables in the model; in other words, one predictor's effect on the DV does not influence the effect of another predictor on the DV. In this study, student pretest scores were included as a predictor variable in the linear regression models used to test hypotheses $3_{\mathbf{a}}$ through $3_{\mathbf{e}}$ and $4_{\mathbf{a}}$ through $4_{\mathbf{e}}$. They were also used to control for initial differences between treatment and control groups. Post hoc independent-samples $t$-tests found that there was a significant difference $(\alpha<.05)$ in mean global awareness pretest scores between students enrolled in a global learning course $(M=1.51, S D=.75)$ and students enrolled in a non-global learning course $(M=1.85, S D=.91) ; t(218)=3.015, p=.003$. There was also a significant difference $(\alpha<.05)$ in mean global perspective pretest scores between students enrolled in a global learning course $(M=.90, S D=.66)$ and students enrolled in a non-global learning course $(M=1.2, S D=.84) ; t(218)=2.987, p=.003$. Since these differences between group pretest scores were associated with the treatment, interaction was tested.

Post hoc tests revealed a significant interaction between students' global awareness pretest scores and the treatment in predicting global awareness posttest scores, $F(3,216)=4.354, p=.005$. Significant interaction was also found between global perspective pretest scores and the treatment in predicting global perspective posttest scores $F(3,216)=4.464, p=.005$. In summary, this study found that for students 
enrolled in global learning courses, those who scored higher on the global awareness and global perspective pretests scored significantly and disproportionately higher on the posttest than did students who received lower pretest scores. These results indicated that the global learning course did indeed have an effect on student achievement of global awareness and global perspective. The rubrics were able to detect the differential effect of the global learning course on student learning, namely that the course significantly increased global awareness and global perspective for students who entered with a minimum level of prior achievement of these outcomes. These minimum levels of prior achievement may be considered measures of students' aptitude or readiness for developing global awareness and global perspective through global learning courses.

These results further substantiate Cronbach's (1957) contention that aptitudetreatment interactions (ATI) frequently underlie educational and psychological testing results. Cronbach based his argument on a number of then-recent studies that uncovered interactions between personality and conditions of learning, interactions that could be used to predict "who will learn better from one curriculum than from the other" (p. 681). What's more, Cronbach argued that in terms of designing treatments to fit individual needs, findings concerning the interaction of attributes with other treatment variables were potentially of greater practical importance than those for which interaction was not uncovered.

One of the principles of ATI is that low structured instructional environments tend to be more conducive to learning for students of higher aptitude or readiness, while highly structured environments may result in better learning for students of lower aptitude or readiness (Cronbach \& Snow, 1977; Snow, 1989). This principle is consistent 
with the study's finding that students of higher aptitude or readiness for global awareness and global perspective were found to learn more in global learning courses than students with lower aptitude or readiness, as reflected in their pretest scores. Global learning courses were characterized by instructional strategies and content associated with ISDs, and the rubrics were designed to measure cognition within ISDs. This study's findings of interaction between pretest scores and treatment thus served as strong evidence of the rubrics' substantive validity as assessments of global awareness and global perspective.

Treatment effect within group. This study involved a within-group discriminant analysis to determine if the global learning course grade could be used to predict a significant amount of the unique variance in student posttest scores. This analysis was conducted in order to gather convergent evidence of the external aspect of the rubrics' construct validity (Messick, 1996). It was assumed that since global learning courses were designed to develop students' global awareness and global perspective, global learning course grades and rubric scores were both measuring the same construct. This study did not reveal a significant relationship between global learning course grades and posttest scores, which may be a source of invalidity for the rubrics. On the other hand, it may be the case that faculty grading criteria and rubric score criteria addressed different constructs, negating the value of this comparison. This study did not collect information concerning the specific criteria used for assigning grades in the global learning courses. Although no significant relationship was found between course grades and the global awareness and global perspective posttest scores, the rubrics were able to detect within-group differences through the interaction between students' pretest scores and treatment. Specifically, students in the treatment group who earned above a certain 
minimum score on the global awareness or global perspective pretest scored significantly and disproportionately higher on the posttest than did those who earned a score below the minimum. This indicates that the rubrics were able to detect differences within groups, strong evidence that they indeed measure the intended constructs.

Relationship between global awareness and global perspective. An additional source of evidence for the rubrics' substantive validity concerned correlations between global awareness and global perspective scores. Post hoc tests revealed a significant moderately positive correlation between global awareness and global perspective pretest scores, $r_{(218)}=.464, p=<.0001$. There was also a significant moderately positive correlation between global awareness and global perspective posttest scores, $r_{(218)}=.462$, $p=<.0001$. These results served as substantive evidence of the rubrics' validity in that they were consistent with one of the theoretical premises of this study, that global awareness and global perspective are distinct yet interrelated outcomes.

Confirmatory evidence for substantive validity reveals "response consistencies or performance regularities reflective of domain processes" (Loevinger, as cited in Messick, 1994, p. 13). Studies examining the relationship of performances across different process models represent a potential empirical source of evidence for substantive validity (Miller \& Linn, 2000). This study found a significant moderately positive relationship between global awareness and global perspective pretest scores as well as between global awareness and global perspective posttest scores. Within the context of global learning, experiences that strengthen one outcome are believed to support the development of the other (Case, 1993; Hanvey, 1975; Merryfield; 2008; Selby \& Pike, 2000). The results of these correlations indicated that the rubrics detected the underlying linear relationship 
between these outcomes, although they could not be used to ascertain a causal relationship between them, as some have theorized (Case, 1993; Hanvey, 1975). The moderate strength of these correlations served as evidence of the rubrics' ability to detect the distinct yet interrelated nature of these outcomes' development. The correlations were not so weak as to cast doubt on an interrelationship, yet not so strong as to imply that the rubrics were actually measuring the same construct.

Extraneous variables. Another of the unexpected results of this study was that with one exception, none of the extraneous variables in the linear models (class status; race/ethnicity; fluency in more than one language; time spent abroad; previous global learning course completion) were found to have accounted for a significant amount of the unique variance in predicting the DVs, global awareness and global perspective posttest scores. The one exception concerned the test for hypothesis $4_{\mathrm{e}}$, in which previous global learning course completion was found to have accounted for a significant amount of unique variance in predicting global perspective posttest scores $(p=.022, \alpha<.05)$. However, the beta coefficient for the variable was negative, meaning that students who took a previous global learning course actually scored lower on the global perspective posttest. This result cast doubt on the confirmation of the hypothesis. Furthermore, all of the regression models yielded low to moderate coefficients of determination, with $R^{2}$ values ranging between .236 and .257 . This suggested that other variables, those not tested in the study, were contributing to the unaccounted for variance in the DVs.

The results from these tests were similar to those of Barrows et al. (1981), who found no relationship between students' educational experiences—coursework, language study, or study abroad — and their levels of knowledge. Barrows et al. theorized that this 
was largely due to deficiencies in students' college education; however, in the present study, the global learning course was found to have a significant albeit differential effect on student learning. Assessment of student attitudes associated with global learning and global citizenship was lacking in both studies. Global education researchers such as Case (1993) and Merryfield (2008) have contended that attitudinal dispositions such as openmindedness, anticipation of complexity, resistance to stereotypes, empathy, and nonchauvinism determine our aptitude or readiness for global awareness and global perspective. Measures of student attitudes may prove to be better predictors of student achievement of these outcomes than the extraneous variables explored in this study.

\section{Study Limitations}

Generalizability of this study to the larger population was limited by the demographic characteristics of the study's sample, which was purposive rather than randomly selected. The study was also limited by attrition bias resulting from students who were absent on the day of the posttest, dropped the course prior to the posttest, or chose not to complete the posttest. Although faculty did not assign credit based on the quality of students' responses, some faculty required assessment completion as part of the course grade while others offered extra credit. Assignment of credit may have influenced students' choice to complete the posttest and/or perform the task to the best of their ability. The study was further limited by the content of the cases that were used. The cases' content was not directly associated with the subject matter of any of the courses involved in the study; therefore, students' background knowledge and/or interest in the cases may have influenced the quality of responses. Additionally, variability in testtaking procedures may have contributed to measurement error that limited the study. 
Faculty allowed approximately 45 minutes to complete the assessment, but this may not have been enough time for some students to complete the task to the best of their ability. Students who came to class late or left early also had less time to complete the task. Other unsystematic errors such as student's test-taking attitudes, motivation, anxiety, and other preconceptions also limited the study (Coaley, 2010).

\section{Implications for Practice}

The results of this study generated practice implications for global learning assessment and instruction. These implications were derived on the basis of their consistency with the results and with literature on global learning and assessment.

\section{Use Rubrics for Formative, Summative, Peer-, and Self-Assessment}

The rubrics used in this study were developed as a pre/post student learning assessment for incoming freshmen, transfers, and graduating seniors participating in a university global learning initiative. Pilot faculty, however, reported that they found the rubrics useful at the classroom level for many types of performance tasks. According to Musil (2006), "the most accessible, and typically the richest, sources of information about student learning are found in the assignments that are an integral part of any course and designed specifically to allow students to demonstrate what they are learning" (p. 20). The global awareness and global perspective rubrics could be used to assess student learning through a wide variety of performance tasks, across the entire span of the curriculum, and at the course, program, and entrance/graduation levels. These multiple formative and summative data sources, using the same evaluative criteria, could help substantiate findings, uncover subtle implications that a single assessment source might miss, identify areas for improvement, and reveal areas for further research (Musil, 2006). 
The rubrics could also be used for valid peer- and self-assessment in order to contribute to increased global learning. When utilized for these purposes, rubrics can be used not only as assessment tools but also as teaching and learning tools (Hafner \& Hafner, 2003). Faculty members sometimes share assessment criteria with students for the sake of fairness and transparency or to encourage students to work towards expectations. Research literature supports the contention that peer- and self-assessment both help students to reflect on the quality of their own performance, benefitting the assessor and the assessee and increasing learning (Lejk \& Wyvill, 2001; Sluijsmans, Moerkerke, van Merrienboer, \& Dochy, 2001). It is recommended here that faculty use the rubrics for peer- and self-assessment to empower and motivate students to actively seek learning experiences that will increase their global awareness and perspective. However, just as the results of this study indicated that training protocols were related to high estimates of reliability, evidence suggests that students should also be trained in the use of relevant rubrics to mitigate error and subjectivity (Sluijsmans, et al., 2001).

\section{Train Faculty and Staff to Integrate Rubrics Into Curriculum and Co-curriculum}

The results of this study demonstrated the importance of theoretical consistency, clear language and construction, and effective training protocols in the development and implementation of valid and reliable rubrics. The methods used in this study may serve as a model for global learning professional development. According to Hurtado (2009), "Institutions must build faculty-driven models of assessment to ensure results will have a direct impact on teaching and learning" (p. 3). Pilot faculty and trained faculty raters involved in this study consistently reflected on the impact of different instructional methods on student achievement as they worked to design and implement a valid and 
reliable assessment instrument for global learning. As a result of their involvement, several faculty members using the instruments customized the language of rubric criteria to address both the global learning outcomes and the specific content of their courses. Student affairs staff could also customize the rubric criteria in order to assess student learning in global learning co-curricular activities. Faculty and staff development could be implemented to increase valid and reliable customization of the global learning rubrics and promote buy-in for assessment.

Professional development could also increase the meaningfulness and utility of the rubrics by encouraging their use for the purpose of Backwards Curriculum Design (Wiggins \& McTighe, 2005). The Backwards Curriculum Design process shifts the educator's perspective away from traditional content coverage towards a learner-centered approach that leads to deeper understanding and critical thinking. Backwards Curriculum Design involves three stages: (a) establishing desired outcomes; (b) determining the kinds of evidence that will demonstrate achievement of the outcomes; and (c) developing learning experiences and selecting content that will enable student achievement of the outcomes. With this shift in perspective, participants see that assessment and teaching strategies are as influential as content. The process of identifying outcomes and developing assessment instruments has been found to positively impact instruction through increased coherence in the organization of curriculum and the use of assessment data for continuous improvement (Crossley \& Wang, 2010).

\section{Use Pretest Data to Differentiate Instruction}

Another implication concerns faculty members utilizing global awareness and global perspective pretest scores to differentiate pedagogy and content in order to 
maximize student learning. Faculty could use enrolled students' pretest averages to make pedagogical decisions concerning the appropriate number and pacing of highly structured vs. low structured learning activities throughout the course and the amount of time allowed for students to complete these tasks (Cronbach \& Snow, 1977; Snow, 1989). Pretest scores could be used to organize diverse learning groups composed of students with varying aptitude or readiness for global awareness and global perspective. To that end, it would be efficacious for global learning faculty to conceive of individual and group learning needs as interrelated. Hanvey (1975) asserted that a global perspective is actually a characteristic of the group, composed of the differentiated cognitive attributes of the individual members of that group. The same may hold true for global awareness, as it is a learning outcome that is highly interconnected with global perspective.

\section{Recommendations for Future Research}

This study adds to the research literature on global learning by providing empirical information regarding the development and validation of rubrics to assess undergraduate students' global awareness and global perspective. In this section, additional research is recommended to expand our knowledge and understanding of such assessments.

\section{Conduct Similar Studies at Other Institutions}

Based upon the results of this study, one recommendation for future research is to replicate the study at other institutions implementing global learning initiatives with similar global learning SLOs. This study was restricted to undergraduate students in selected global learning and non-global learning courses at one large, Hispanic-serving, 
urban, public, research university in South Florida. Results may differ at other types of institutions, in different courses, with dissimilar student demographics.

\section{Apply Rubrics to Other Performance Assessments}

In this study the global awareness and global perspective rubrics were used to assess student learning through one kind of performance task, written responses to two open-ended questions concerning complex case studies. In addition, the content of the cases used was not aligned with the subject matter of any of the courses involved in the study. Since this study required an instrument that could be used across the curriculum, it was decided to utilize cases that could be understood via numerous disciplinary perspectives. Many institutions are in need of cross-disciplinary instruments in order to conduct comparisons within and between institutions for the purpose of continuous improvement (Musil, 2006; Sternberger, Pysarchik, Yun, \& Deardorff, 2009). However, studies are needed to determine if valid, reliable comparisons can be made using uniform global learning performance criteria but differentiated and discipline-specific authentic performance tasks, such as portfolios, research papers, poster presentations, case studies, speeches, debates, films, blogs, models and prototypes, and fine and performing arts presentations.

\section{Conduct Qualitative and Mixed Methods Studies}

Further research concerning the validity and reliability of the global awareness and global perspective rubrics should involve qualitative and mixed-methods approaches that make use of evidence sources that differ from those explored in this study. Among these studies, other IVs should be explored for their contribution to the unique variance of student scores. These may include critical thinking skills, attitudes towards global 
learning, and dispositions that may determine aptitude or readiness for global awareness and global perspective, such as openmindedness, anticipation of complexity, resistance to stereotypes, empathy, and non-chauvinism (Case, 1993; Merryfield, 2008).

Studies should also gather additional evidence concerning the rubrics' reliability through test-retest studies of intra-rater reliability. To bolster evidence of their validity, content analysis of the rubrics and performance tasks should be conducted to determine the extent to which they adequately address the constructs of global awareness and global perspective. Think-aloud interviews could also be conducted to substantiate the substantive validity of the rubrics' scoring level criteria and model responses. Since the rubrics' scoring scale and criteria follow a continuum grounded in Bloom's (1956) Taxonomy of Cognitive Development, rubric scores could be compared to those yielded from other assessments of critical thinking in order to gather convergent evidence of the external aspect of the rubrics' construct validity. Studies are also needed concerning the consequential aspect of the rubrics' construct validity. Experimental and naturalistic explorations of the rubrics' meaningfulness and utility for faculty, staff, administrators, and students, as well as intended and unintended consequences of their use and interpretation, would provide a valuable contribution to the literature on valid global learning assessment.

\section{Conduct Long-term Studies}

This pretest/posttest study compared the average learning gains of students enrolled in global learning courses and non-global learning courses over the course of a single semester. It may be the case that this span of time was too brief for many students to make measurable, much less significant, strides in their development of global 
awareness and global perspective. Spiro et al. (1988) have contended that in ISDs, exposure to multiple case studies over time is necessary for students to overcome overreliance on reductive cognitive schema. Hanvey (1975) also argued that individuals develop a suite of attributes associated with a global outlook to a greater or lesser degree throughout the course of their lives. Long-term studies utilizing empirical and qualitative evidence are therefore needed to gauge students' learning after taking multiple global learning courses or after having engaged in multiple opportunities to apply their global awareness and global perspective in the undergraduate curriculum and co-curriculum.

\section{Conclusions}

While colleges and universities across the United States have surged forward in their implementation of a variety of global learning initiatives, effective student learning assessment of these programs has lagged behind (Grudzinski-Hall, 2007). Institutions are in need of valid and reliable assessments of global learning outcomes, such as global awareness and global perspective, in order to make valid data-based decisions that improve student learning through curriculum, faculty development and placement, planning, and budgeting. In many cases, institutions must provide student learning evidence to accrediting agencies, students, and other stakeholders that demonstrates the extent to which such decisions are meaningful, useful, and appropriate (Messick, 1998). These student learning-based decisions have ethical, instructional, and practical implications that ultimately influence the efficacy of the educational endeavor.

This study examined the development of rubrics to measure undergraduate students' global awareness and global perspective at one large, Hispanic-serving, urban, public, research university in South Florida. It was undertaken to demonstrate the extent 
to which evidence supported the validity and reliability of scores yielded from the rubrics.

The results of this study suggested that the rubrics were highly reliable and could be used to validly detect the differentiated effects of global learning courses on student development of global awareness and global perspective. Although these particular rubrics may not be appropriate for use at all universities implementing global learning initiatives, this study's methods could be used as a model for other institutions to leverage scarce internal resources in order to gather valid, reliable student learning data to facilitate self-improvement and address the exigencies of external accountability. 


\section{REFERENCES}

Adams, J. M., \& Carfagna, A. (2006). Coming of age in a globalized world. Bloomfield, IL: Kumarian Press.

American Association of Colleges and Universities. (2005). Globalizing the curriculum. Diversity Digest, 8(3), 12.

American Association for Higher Education. (1991). Nine principles of good practice for assessing student learning. Sterling, VA: Stylus.

American Council on International Intercultural Education Conference. (1996, November). Educating for the global community, a framework for community colleges. Paper presented at the Stanley Foundation and the American Council on International Intercultural Education Conference, Warrenton, VA. Retrieved from www.stanleyfoundation.org/publications/archive/CC2.pdf

American Educational Research Association, American Psychological Association \& National Council on Measurement in Education. (1999). Standards for educational and psychological testing. Washington, DC: American Educational Research Association.

Anderson, C. C., Nicklas, S. K., \& Broadford, A. R. (1994). Global understandings: A framework for teaching and learning. Retrieved from ERIC database. (ED381462)

Anderson, H. R. (1954). Developing an understanding of world affairs. In H. R. Anderson (Ed.)., Approaches to an understanding of world affairs (pp. 1-10). Menasha, WI: George Banta Publishing.

Anderson, L. F. (1979). Schooling and citizenship in a global age: An exploration of the meaning and significance of global education. Retrieved from ERIC database. (ED214834)

Andrade, H. G. (2005). Teaching with rubrics: The good, the bad, and the ugly. College Teaching, 53(1), 27-30.

Appiah, K. A. (2006). Cosmopolitanism: Ethics in a world of strangers. New York, NY: W. W. Norton \& Company.

Archbald, D.A., \& Newmann, F.M. (1988). Beyond standardized testing: Assessing authentic academic achievement in the secondary school. Reston, VA: National Association of Secondary School Principals. 
Arum, S., \& Van de Water, J. (1992). The need for definition of international education in U.S. universities. In C. B. Klasek (Ed.), Bridges to the future: Strategies for internationalizing higher education (pp. 191-203). Carbondale, IL: Association of International Education Administrators.

Auba, J. (1970). Comenius and the organisation of education. In C. H. Dobinson (Ed.), Comenius and contemporary education (pp. 60-75). Retrieved from ERIC database. (ED079212)

Banks, J. A. (2008). Diversity, group identity, and citizenship education in a global age. Educational Researcher, 37(3), 129-139.

Barrows, T. S., Ager, S. M., Bennett, M. F., Braun, H. I., Clark, J. L. D., Harris, L. G., \& Klein, S. F. (1981). College students' knowledge and beliefs: A survey of global understanding. New Rochelle, NY: Change Magazine Press.

Barrows, T. S., Clark, J., \& Klein, S. (1980). What students know about their world. Change, 12(4), 10-17.

Becker, J. M. (1969). An examination of objectives, needs and priorities in international education in U.S. secondary and elementary schools. Retrieved from ERIC database. (ED031612)

Bennett, M. J. (1986). A developmental approach to training for intercultural sensitivity. International Journal of Intercultural Relations, 10(2), 179-196.

Biggs, J. (1999). Enhancing teaching through constructive alignment. Higher Education, $32(3), 347-364$.

Bloom B. S. (1956). Taxonomy of educational objectives, handbook I: The cognitive domain. New York, NY: David McKay.

Bonney, C. G. (1894). Address of welcome. Proceedings of the International Congress of Education of the Columbian Exposition, Chicago, July 25-28, 1893. New York, NY: National Education Association.

Bonwell, C. C., \& Eison, J. A. (1991). Active learning: Creating excitement in the classroom. Retrieved from ERIC database. (ED336049)

Bowen, L. (2005). Engaging diversity on the homogenous campus: The power of immersion experiences. Diversity Digest, 8(3), 18-19.

Bragaw, D. (2001). Schooling and citizenship in a global age. Retrieved from ERIC database. (ED460055) 
Braskamp, L. (2010). Being effective interventionists to foster global citizenship. Journal of College and Character, 11(1), 1-6.

Braskamp, L., Braskamp, D. C., \& Merrill, K. C. (2009). Global perspectives inventory (GPI): Its purpose, construction, potential uses, and psychometric characteristics. Retrieved from https://gpi.central.edu/supportDocs/manual.pdf

Brunold, A. O. (2005). Global learning and education for sustainable development. Higher Education in Europe, 30(3 \& 4), 295-306.

Buell, R. L. (1925). International relations. New York, NY: Henry Holt and Company.

Burn, B. B. (1980). Expanding the international dimension of higher education. London, England: Jossey-Bass Publishers.

Butts, R. F. (1969). America's role in international education: A perspective on thirty years. In H. G. Shane (Ed.), The United States and international education: The sixty-eighth yearbook of the National Society for the Study of Education (pp. 345). Chicago, IL: University of Chicago Press.

Butts, R. F. (1971). International education: overview. In L. C. Deighton (Ed.), The encyclopedia of education (pp. 164-171). New York, NY: Macmillan.

Campbell, D. T., \& Stanley, J. C. (1963). Experimental and quasi-experimental designs for research. Chicago, IL: Rand McNally.

Case, R. (1993). Key elements of a global perspective. Social Education, 57(6), 318-325.

Castells, M. (1999). Information technology, globalization, and social development [PDF document]. Discussion paper, United Nations Research Institute for Social Development. Retrieved from http://vega.soi.city.ac.uk/ abct353/SM1061N/Castells_1999.pdf

Chase-Dunn, C. (1999). Globalization: A world-systems perspective. Journal of WorldSystems Research, 2(3), 187-215.

Chickering, A., \& Braskamp, L. (2009). Developing a global perspective for personal and social responsibility. Peer Review, 11(4), 27-30.

Clark, J. L. D. (1981). Language. In T. S. Barrows, S. M. Ager, M. F. Bennett, H. I. Braun, J. L. D. Clark, L. G. Harris, \& S. F. Klein, College students' knowledge and beliefs: A survey of global understanding. New Rochelle, NY: Change Magazine Press. 
Clark, R. P. (2002). Global awareness: Thinking systematically about the world. Lanham, MD: Rowman and Littlefield.

Clarke, V. (2004). Students' global awareness and attitudes to internationalism in a world of cultural convergence. Journal of Research in in International Education, 3(1), 51-70.

Coaley, K. (2010). An introduction to psychological assessment and psychometrics. London, England: Sage.

Cohen, J., Cohen, P., West, S. G., \& Aiken, L. S. (2003). Applied multiple regression/correlation analysis for the behavioral sciences. Mahwah, NJ: Lawrence Erlbaum Associates.

Cohen, J. (1988). Statistical power analysis for the behavioral sciences (2nd edition). Hillsdale, NJ: Lawrence Erlbaum Associates, Inc.

Cole, K. (2003). Globalization: Understanding complexity. Progress in Development Studies, 3(4), 323.

Cook, T.D., \& Campbell, D.T. (1979). Quasi-experimentation: design and analysis issues for field settings. Chicago, IL: Rand McNally.

Cornwell, G. H., \& Stoddard, E. W. (1999). Globalizing knowledge: Connecting international and intercultural studies. Washington, DC: American Association of Colleges and Schools.

Cronbach, L. J. (1957). The two disciplines of scientific psychology. American Psychologist, 12(11), 671-684.

Cronbach, L. J., \& Snow, R. (1977). Aptitudes and instructional methods: A handbook for research on interactions. New York, NY: Irvington Publishers, Inc.

Crossley, M., \& Wang, L. (2010). Learning by doing: An experience with outcomes assessment. The University of Toledo Law Review, 41(2), 269-292.

Davies, L. (2006). Global citizenship: Abstraction or framework for action? Educational Review, 58(1), 5-25.

Davis, N. J., \& Robinson, R. V. (2006). Using a research article to foster moral reflection and global awareness in teaching about religion and politics, theory testing, and democracy in the Muslim world. Teaching Sociology, 34(3), 296-312.

Donnelly-Smith, L. (2009). Global learning through short-term study abroad. Peer Review, 11(4), 12-15. 
Drucker, P. (1999). Beyond the information revolution. Atlantic Monthly, 284(3), 47-48.

Education Development Center. (2006, September 19). Global youth survey explores perspectives on social, cultural identity. Retrieved from http://www.edc.org/newsroom/press_releases/global_youth_survey_explores_pers pectives_social_cultural_identity

Faure, E., Herrera, F., Kaddoura, A-R., Lopes, H., Petrovsky, A. V., Rahnema, M., \& Ward, F. C. (1972). Learning to be: The world of education today and tomorrow. Paris, France: United Nations Educational, Scientific and Cultural Organization.

Falk, R. (1994). The making of global citizenship. In B. V. Steenbergen (Ed.), The condition of citizenship (pp. 127-140). London, England: Sage Publications.

Fleenor, J. W., Fleenor, J. B., \& Grossnickle, W. F. (1996). Interrater reliability and agreement of performance ratings: A methodological comparison. Journal of Business and Psychology, 10(3), 367-380.

Florida International University. (2010, September). Florida International University's quality enhancement plan: Global Learning for Global Citizenship. Retrieved from http://goglobal.fiu.edu/QEP_Report-Final.pdf

Fraser, S. E., \& Brickman, W. W. (1968). A history of international and comparative education: Nineteenth century documents. Glenview, IL.: Scott, Foresman.

Gaudelli, W. (2003) World class: Teaching and learning in global times. Mahwah, NJ: Lawrence Erlbaum Associates, Inc.

Gearhart, M. (1994). Toward the instructional utility of large-scale writing assessment: Validation of a new narrative rubric. Retrieved from ERIC database. (ED379294)

Gibson, K. L., Rimmington, G. M., \& Landwehr-Brown, M. (2008). Developing global awareness and responsible world citizenship with global learning. Roeper Review, $30(1), 11-23$.

Gilliom, E. M. (2001). Global education and the social studies. Theory Into Practice, 20(3), 169-173.

Golich, V. L., Boyer, M., Franko, P., \& Lamy, S. (2000). The ABCs of case teaching. Retrieved from http://ecase.georgetown.edu/abcs.pdf

Good, H. G. (1960). A history of western education. New York, NY: Macmillan. 
Greater Expectations Project on Accreditation and Assessment. (2004). Taking responsibility for the quality of the baccalaureate degree. Retrieved from http://www.mus.edu/transfer/councils/TakingResponsibility.pdf

Green, S. B. (1991). How many subjects does it take to do a regression analysis? Multivariate Behavioral Research, 26, 499-510.

Grudzinski-Hall, M. N. (2007). How do college and university undergraduate level global citizenship programs advance the development and experiences of global competencies? Retrieved from Dissertations \& Theses: Full Text. (AAT 3261868)

Hafner, J. C., \& Hafner, P. M. (2003). Quantitative analysis of the rubric as an assessment tool: An empirical study of student peer-group rating. International Journal of Science Education, 25(12), 1509-1528.

Hammer, M. R., Bennett, M. J., \& Wiseman, R. (2003). The Intercultural Development Inventory: A measure of intercultural sensitivity. International Journal of Intercultural Relations, 27(4), 421-443.

Hanvey, R. G. (1975). An attainable global perspective. Retrieved from ERIC database. (ED116993)

Herman, J. L., Aschbacher, P. R., \& Winters, L. (1992). Using alternative assessment for decision making. A practical guide to alternative assessment. Alexandria, VA: Association for Supervision and Curriculum Development.

Hett, E. J. (1993). The development of an instrument to measure global-mindedness. (Doctoral dissertation). Retrieved from ProQuest dissertations \& theses. (Publication No. AAT 9408210).

Hicks, D. (2003). Thirty years of global education: A reminder of key principles and precedents. Educational Review, 55(3), 265-275.

Holden, C. (2000). Learning for democracy: From world studies to global citizenship. Theory Into Practice, 39(2), 74-80.

Hovland, K. (2006). Shared futures: Global learning and liberal education. Washington, DC: Association of American Colleges and Universities.

Hovland, K. (2009). Global learning: What is it? Who is responsible for it? Peer Review, 11(4), 4-7.

Howard, E. R. (2002). Two-way immersion: A key to global awareness. Educational Leadership, 60(2), 62-64. 
Huba, M. E., \& Freed, J. E. (2000). Learner-centered assessment on college campuses: Shifting the focus from teaching to learning. Boston, MA: Allyn and Bacon.

Hunter, B., White, G. P., \& Godbey, G. C. (2006). What does it mean to be globally competent? Journal of Studies in International Education, 10(3), 267-285.

Hurtado, S. (2009). Assessing higher education's advancement toward a new vision of society. Diversity \& Democracy, 12(1), 1-3.

International Association of Universities. (2003). Internationalization of higher education: Trends and developments since 1998. Retrieved from http://portal.unesco.org/education/en/files/20030/10561413171IAU_rev.pdf/IAU_ rev.pdf

Joint Committee on Testing Practices. (2004). Code of fair testing practices in education. Retrieved from http://www.ncme.org/pubs/pdf/CodeofFairTestingPractices.pdf

Johnson, R. L., Penny, J., \& Gordon, B. (2000). The relation between score resolution methods and interrater reliability: An empirical study of an analytic scoring rubric. Applied Measurement in Education, 13(2), 121-138.

Jonsson, A., \& Svingby, G. (2007). The use of scoring rubrics: Reliability, validity and educational consequence. Educational Research Review, 2(2), 130-144.

Kandel, I. L. (1937). Intelligent nationalism in the school curriculum. In I. L. Kandel \& G. M. Whipple (Eds.), Thirty-sixth yearbook of the National Society for the Study of Education, committee on understanding, Part II, international understanding through the public school curriculum (pp. 35-42). Bloomington, IN: Public School Publishing, Inc.

Kandel, I. L. (1955). National and international aspects of education. International Review of Education, 1(1), 5-15.

Keeling, R. P. (2004). Learning reconsidered. Washington, DC: National Association of Student Personnel Administrators.

Kenworthy, L. S. (1951). The schools of the world an education for a world society. In C. O. Arndt and S. Everett (Eds.), Education for a world society (pp. 199-230). New York, NY: Harper \& Brothers.

Keohane, R. O., \& Nye, J. S., Jr. (2000). Globalization: What's new? what's not? (and so what?). Foreign Policy, 118, 104.

Kerr, C. (1979). Education for global perspectives. The Annals of the American Academy of Political and Social Science, 442(1), 109-116. 
Kirkwood, T. F. (2001). Our global age requires global education: Clarifying definitional ambiguities. The Social Studies, 92(1), 10-15.

Kniep, W. M. (1989). Essentials for a global education. The ATA Magazine, 69(4), 12-15.

Lamy, S. (1982). Teacher training in global perspectives education: The center for teaching international relations. Theory Into Practice, 21(3), 206-211.

Lamy, S. (2007). Challenging hegemonic paradigms and practices: Critical thinking and active learning strategies for international relations. PS: Political Science and Politics, 40(1), 112-116.

Landis, D., Bennett, J. M., \& Bennett, M. J. (2004). Handbook of intercultural training ( $3^{\text {rd }}$ ed. $)$. Thousand Oaks, CA: Sage Publications, Inc.

Lantis, J. S., Kille, K. J., \& Krain, M. (2010). The state of the active teaching and learning literature. In R. A. Denemark (Ed.), The international studies encyclopedia. Chichester, West Sussex, England: Wiley-Blackwell.

Lemke, C. (2002). EnGauge $21^{\text {st }}$ century skills: Digital literacies for a digital age. Retrieved from ERIC database. (ED463753)

Loevinger, J. (1957). Objective tests as instruments of psychological theory. Psychological Reports, 3(3), 635-694.

Library of Congress Authorities. (2012). International education. Retrieved from http://authorities.loc.gov/

Loveland, E. (2010). Global learning for all. International Educator, 19(6), 12-23.

Marascuilo, L. A., \& Levin, J. R. (1970). Appropriate post hoc comparisons for interaction and nested hypotheses in analysis of variance designs: The elimination of Type IV errors. American Educational Research Journal, 7(3), 397-421.

McNeil, K. A., Newman, I., \& Fraas, J. W. (2012). Designing general linear models to test research hypotheses. Lanham, MD: University Press of America, Inc.

McTighe, J., \& Thomas, R. S. (2003). Backward design for forward action. Educational Leadership, 60(5), 52-55.

Merryfield, M. (2008). Scaffolding social studies for global awareness. Social Education, 72(7), 363-366.

Messick, S. (1989). Validity. In R. L. Linn (Ed.), Educational measurement (3rd ed., pp.13-103). New York, NY: American Council on Education/ Macmillan. 
Messick, S. (1994). Validity of psychological assessment: Validation of inferences from persons' responses and performances as scientific inquiry into score meaning. Retrieved from ERIC database. (ED380496)

Messick, S. (1996). Validity of performance assessments. In G.W. Phillips (Ed.), Technical issues in large scale performance assessment (pp. 1-18). Washington, DC: National Center for Educational Statistics.

Messick, S. (1998). Test validity: A matter of consequence. Social Indicators Research, $45(1), 35-44$.

Miller, D. M., \& Linn, R. L. (2000). Validation of performance-based assessments. Applied Psychological Measurement, 24(4), 367-378.

Moore, J. R. (2008). The role of ethnicity in social studies education: Identity and conflict in a global age. Social Studies Research and Practice, 3(1), 42-54.

Moskal, B. M. (2000). Scoring rubrics: What, when and how? Practical Assessment, Research \& Evaluation. 7(3). Retrieved from http://PAREonline.net/getvn.asp?v=7\&n=3

Moskal, B. M., \& Leydens, J. A. (2000). Scoring rubric development: validity and reliability. Practical Assessment, Research \& Evaluation, 7(10). Retrieved from http://PAREonline.net/getvn.asp? $=7 \& \mathrm{n}=1$

Murray, S. G. (1929). The international aspect of education. School and Society, 30(764), 213-215.

Musil, C. M. (2006). Assessing global learning: Matching good intentions with good practice. Washington, DC: Association of American Colleges and Universities.

National Leadership Council for Liberal Education \& America's Promise (LEAP). (2007). College learning for the new global century [PDF document]. Retrieved from http://www.aacu.org/leap/documents/GlobalCentury_final.pdf

Newman, I., Newman, C, Brown, R., \& McNeely, S. (2006). Conceptual statistics for beginners (3rd ed.). Lanham, MD: University Press of America.

Nussbaum, M. (2004). Liberal education and global community. Liberal Education, 90(4), 42-48.

Our World Alliance. (2006). Survey of youth identity and citizenship. Retrieved from http://www.jugendhilfeportal.de/db/adminbin/getfile.php?c_fileid=DE0260000006 
Palm, T. (2008). Performance assessment and authentic assessment: A conceptual analysis of the literature. Practical Assessment, Research \& Evaluation, 13(4). Retrieved from $\mathrm{http} / / /$ pareonline.net/genpare.asp? $\mathrm{wh}=0 \& \mathrm{abt}=13$

Piaget, J. (1993). John Amos Comenius. Prospects, 23(1/2), 173-196.

Pike, G., \& Selby, D. (2001). In the global classroom, 2. Toronto, Canada: Pippin Publishing.

Popham, W. J. (1997). What's wrong —and what's right—with rubrics. Educational Leadership, 55(2), 72-75.

Popp, S. E. O., Ryan, J. M., \& Thompson, M. S. (2009). The critical role of anchor paper selection in writing assessment. Applied Measurement in Education, 22(3), 255271.

Prescott, D. (1930). Education and international relations: A study of the social forces that determine the influence of education. Cambridge, MA: Harvard University Press.

Rivas, O., Jones, I., \& Pena, E. (2010). Lessons learned while conducting educational program assessment. Journal of Case Studies in Accreditation and Assessment, 1, $1-8$.

Roeper, A. (1992). Global awareness and the young child. Roeper Review, 15(1), 52-53.

Roeper, A. (2008). Global awareness and gifted children. Roeper Review, 30(1), 8-10.

Sadler, F. E. (1970). Comenius as an international citizen. In C. H. Dobinson (Ed.), Comenius and contemporary education (pp. 60-75). Retrieved from ERIC database. (ED079212)

Sampson, D. \& Smith, H. (1957). A scale to measure world-minded attitudes. Journal of Social Psychology, 45(1), 99-106.

Scanlon, D. G. (1960). International education: An introduction. In D. G. Scanlon (Ed.), International education: A documentary history. New York, NY: Columbia University, Bureau of Publications, Teachers College.

Selby, D., \& Pike, G. (2000). Civil global education: Relevant learning for the twentyfirst century. Convergence, 33(1/2), 138-149.

Simonson, M. R. (1977). Global awareness: A curriculum plan for world study. National Association of Secondary School Principals Bulletin, 61(411), 75-80. 
Skelton, M. (2010). Global learning. Education Journal, 124, 39.

Sluijsmans, D. M. A., Moerkerke, G., van Merrienboer, J. J. G., \& Dochy, F. J. R. C. (2001). Peer assessment in problem based learning. Studies in Educational Evaluation, 27(2), 153-173.

Snow, R. (1989). Aptitude-treatment interaction as a framework for research on individual differences in learning. In P. Ackerman, R.J. Sternberg, \& R. Glaser (Ed.), Learning and Individual Differences. New York, NY: W.H. Freeman.

Sobania, N., \& Braskamp, L. (2009). Study abroad or study away: It's not merely semantics. Peer Review, 11(4), 17-20.

Spiro, R. J., Coulson, R. L., Feltovich, P. J., \& Anderson, D. (1988). Cognitive flexibility theory: Advanced knowledge acquisition in ill-structured domains. Retrieved from ERIC database. (ED302821)

Spiro, R. J. \& Deschryver, M. (2009). Constructivism: When it's the wrong idea and when it's the only idea. In S. Tobias \& T.M. Duffy (Eds.), Constructivist instruction: Success or failure? New York: Routledge.

Spiro, R. J., Feltovich, P. J., Jacobson, M. I., \& Coulson, R. L. (1995). Cognitive flexibility, constructivism, and hypertext: Random access instruction for advanced knowledge acquisition in ill-structured domains. In L. P. Steffe \& J. E. Gale (Eds.), Constructivism in education (pp. 85-107). Mahwah, NJ: Lawrence Erlbaum Associates, Inc.

Spiro, R. J., Vispoel, W. L., Schmitz, J., Samarapungavan, A., \& Boerger, A. (1987). Knowledge acquisition for application: Cognitive flexibility and transfer in complex content domains. Retrieved from ERIC database. (ED287155)

Steen, L. (1999). Assessing assessment. In B. Gold, S. Z. Keith \& W. A. Marion (Eds.), Assessment practices in undergraduate mathematics (pp. 1-5). Washington, DC: Mathematical Association of America.

Steenburgen, B. V. (1994). The condition of citizenship. London, England: Sage.

Stellmack, M. A., Konheim-Kalkstein, Y. L., Manor, J. E., Massey, A. R., \& Schmitz, J. A. P. (2009). An assessment of reliability and validity of a rubric for grading APA-style introductions. Teaching of Psychology, 36(2), 102-107.

Sternberger, L., Pysarchik, D. T., Yun, Z., \& Deardorff, D. (2009). Designing a model for international learning assessment. Diversity \& Democracy, 12(1), 7-9. 
Stoker, S. (1933). The schools and international understanding. Chapel Hill, NC: University of North Carolina Press.

Stomfay-Stitz, A. M. (1993). Peace education in America, 1828-1990: Sourcebook for education and research. London, England: Scarecrow Press.

Sylvester, R. (2002). Mapping international education: A historical survey, 1893-1944. Journal of Research in International Education, 1(1), 90-125.

Sylvester, R. (2003). Further mapping of the territory of international education in the $20^{\text {th }}$ century (1944-1969). Journal of Research in International Education, 2(2), 185-204.

Sylvester, R. (2005). Framing the map of international education (1969-1998). Journal of Research in International Education, 4(2), 123-151.

Temple-Thurston, B. (2005). Student civic engagement at home and abroad. Diversity Digest, 8(3), 8-9.

Tewksbury, D. G. (1945). New directions in international education. Teachers College Record, 46(5), 293-301.

Thaler, N., Kazemi, E., \& Huscher, C. (2009). Developing a rubric to assess student learning outcomes using a class assignment. Teaching of Psychology, 36(2), 113116.

Thompson, J. B. (2003). The globalization of communication. In D. Held and A. McGrew (Eds.), The global transformations reader (pp. 246-259). Cambridge, England: Polity Press.

Tierney, R., \& Simon, M. (2004). What's still wrong with rubrics: Focusing on the consistency of performance criteria across scale levels. Practical Assessment, Research \& Evaluation, 9(2). Retrieved from http://PAREonline.net/getvn.asp? $\mathrm{v}=9 \& \mathrm{n}=2$

Tochon, F. V. (2009). The key to global understanding: World languages educationwhy schools need to adapt. Review of Educational Research, 79(2), 650-681.

Tomlinson, J. (1999). Globalization and culture. Chicago, IL: University of Chicago Press.

Torney-Purta, J. V. (1982). The global awareness survey: Implications for teacher education. Theory Into Practice, 21(3), 200-205. 
Torney-Purta, J. V. (1989). A research agenda for the study of global/international education in the United States. Retrieved from ERIC database. (ED313295)

Tucker, J. L. (1982). Developing a global dimension in teacher education: The Florida International University experience. Theory Into Practice, 21(3), 212-217.

Tye, B. B., \& Tye, K. A. (1992). Global education: A study of school change. Albany, NY: State University of New York Press.

Tye, K. A. (1990). Introduction: The world at a crossroads. In K. A. Tye (Ed.), Global education: From thought to action (pp. 1-9). Alexandria, VA: The Association for Supervision and Curriculum Development.

Tye, K. A. (2009). A history of the global education movement in the United States. In T. F. Kirkwood-Tucker (Ed.), Visions in global education: The globalization of curriculum and pedagogy in teacher education and schools (pp. 3-24). New York, NY: Peter Lang Publishing, Inc.

Van Voorhis, C. R. W., \& Morgan, B. L. (2007). Understanding power and rules of thumb for determining sample sizes. Tutorials in Quantitative Methods for Psychology, 3(2), 43-50.

Volkwein, J. F. (2009). The assessment context: Accreditation, accountability, and performance. New Directions for Institutional Research, 2010(S1), 3-12.

Von Karolyi, C. (2008). Introduction to special issue on global awareness and giftedness. Roeper Review, 30(1), 6-7.

Wicklund, R. A. Multiple perspectives in person perception and theorizing. Theory \& Psychology, 9(5), 668-678.

Wiggins, G. (1990). The case for authentic assessment. Practical assessment, research \& evaluation, 2(2). Retrieved from http://PAREonline.net/getvn.asp? $\mathrm{v}=2 \& \mathrm{n}=2$

Wiggins, G. (1993). Assessment: Authenticity, context, and validity. Phi Delta Kappan, 75(3), 200-214.

Wiggins, G., \& McTighe, J. (2005). Understanding by design. Alexandria, VA: Association for Supervision and Curriculum Development.

Wilson, H. E., \& Collings, M. R. (1963). Education for international understanding. National Elementary Principal, 42(6), 11-17. 
Wolkonsky, S. (1894). Address of welcome by the delegate of the ministry of public education in Russia. Proceedings of the International Congress of Education of the world's Columbian exposition in Chicago, July 25-28, 1893 (pp. 37-9). New York: National Education Association.

Wooten, F. (1929). The international school of Geneva. School and Society, 30(758), 2325.

Wraga, W. G. (2008). Trying our own medicine: The case method of instruction in the United States in historical perspective. International Journal of Case Method Research \& Application, 20(3), 299-312. 
APPENDICES 
APPENDIX A

Blank Global Learning Course Assessment Plan 


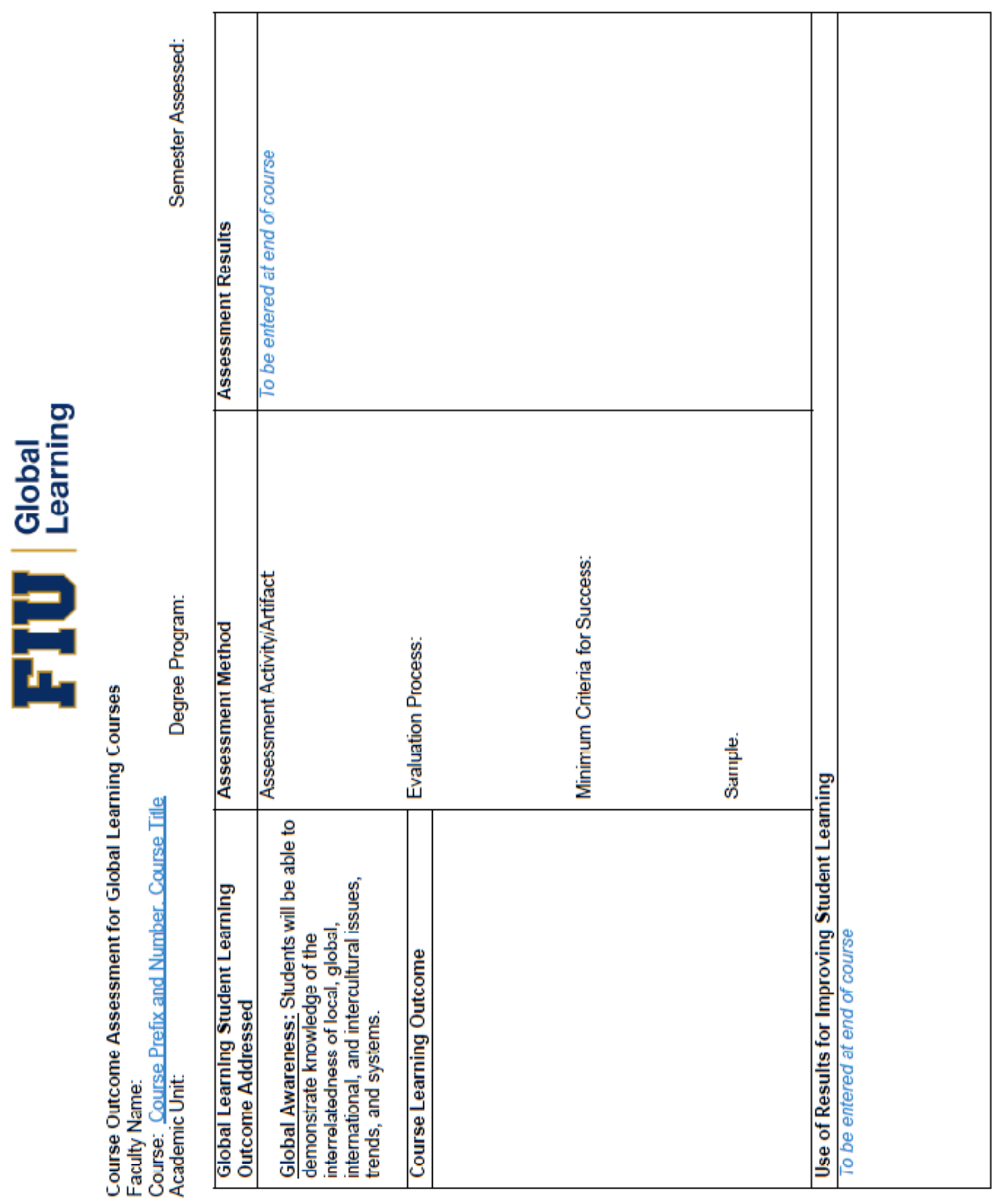




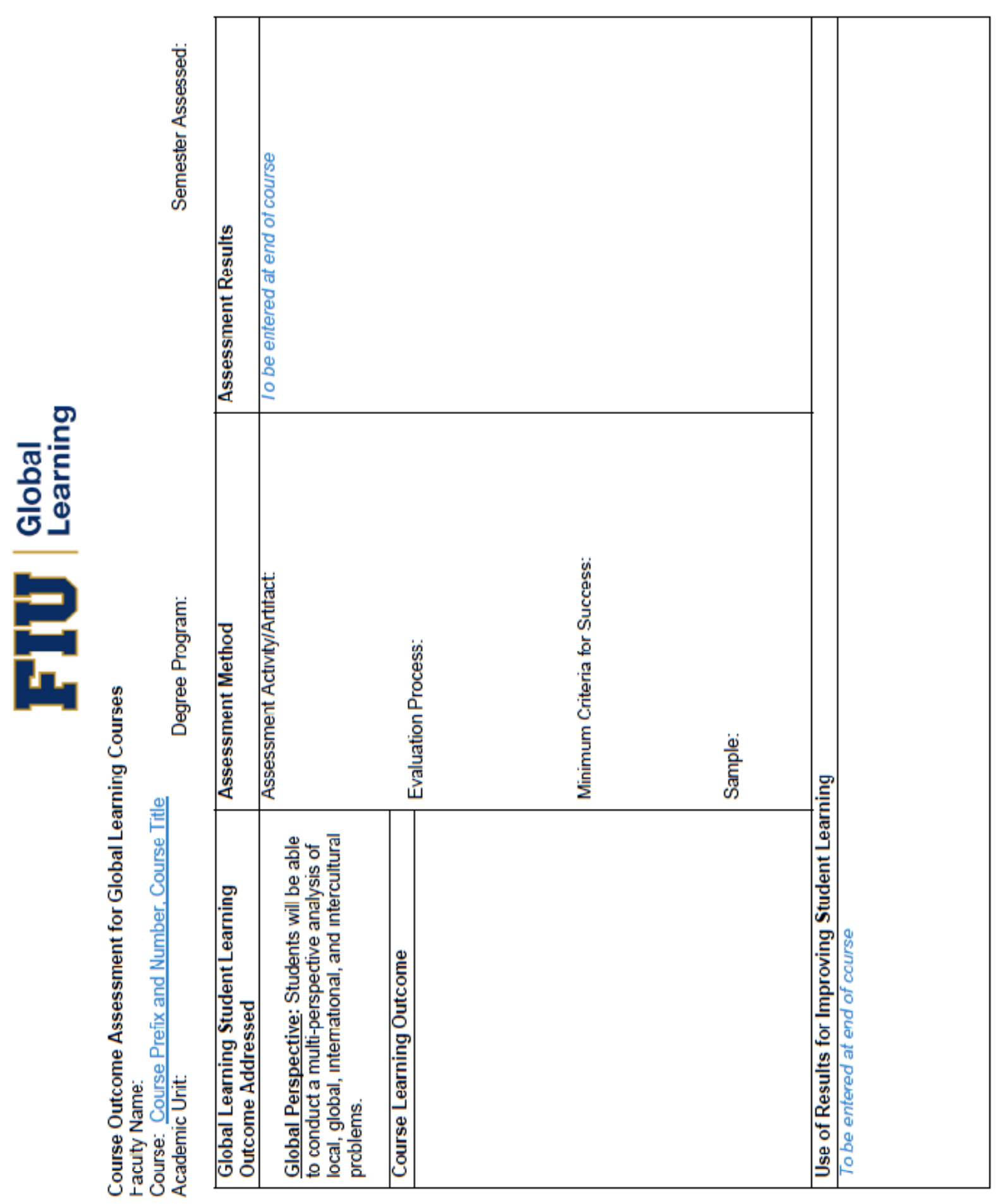




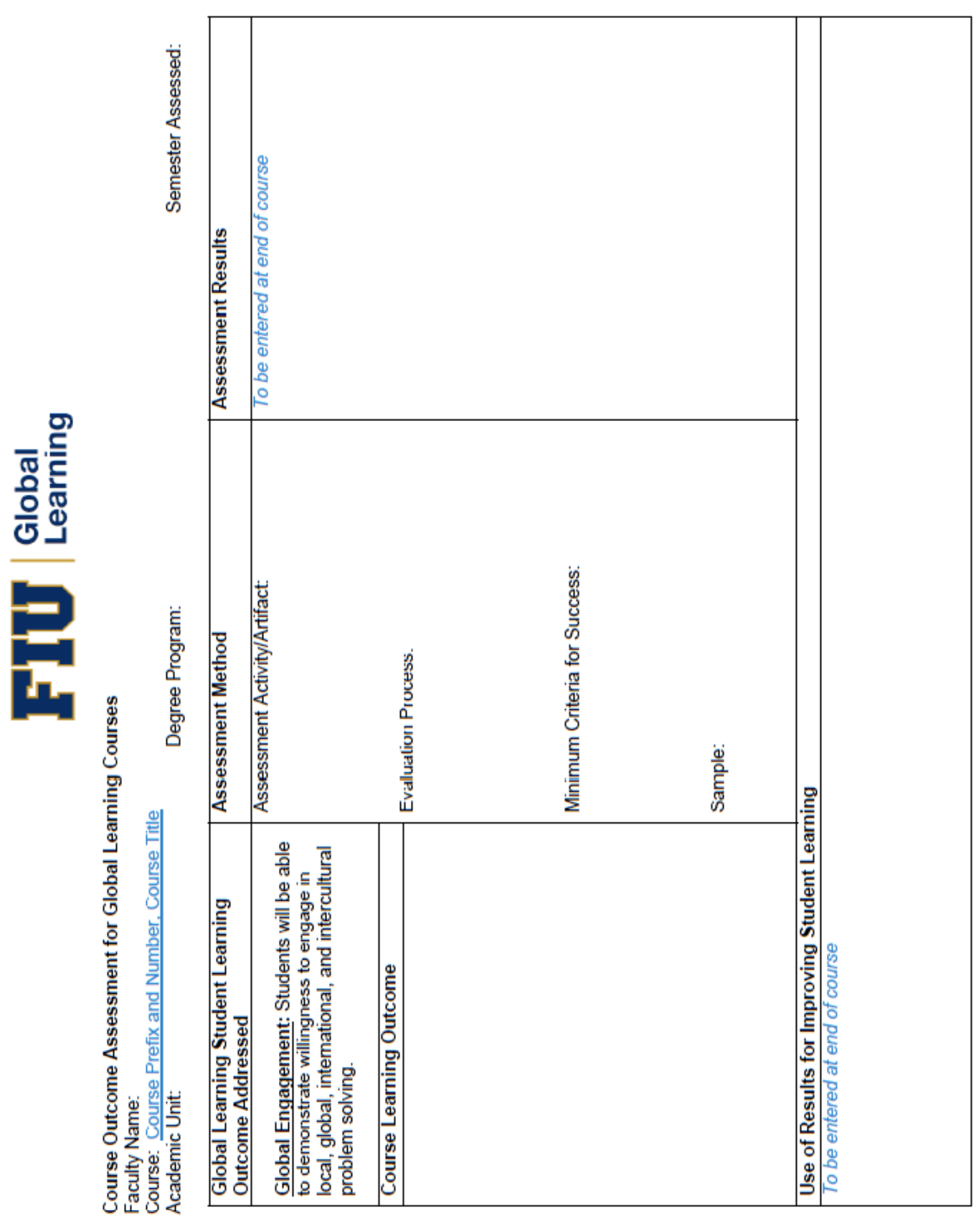


APPENDIX B

Global Learning Foundations Course Approval Guidelines 


\section{GLOBAL LEARNING FOUNDATIONS COURSE APPROVAL CHECKLIST}

1. Does the course state the global learning student learning outcomes?

The course outcomes must address both the global learning student learning outcomes and the course content. The outcomes must be measurable and address higher order thinking skills (analysis, synthesis, and evaluation).

2. Does the course include a comprehensive assessment plan for the global learning student learning outcomes?

The assessment plan for the global learning course outcomes must include a description of appropriate assessment artifacts, evaluation processes, minimum criteria for success, and sampling methods.

3. Does the course address the global learning course outcomes through appropriate interdisciplinary content and readings?

The course must address essential questions associated with global, local, international, and/or intercultural issues and/or problems. Students must engage in interdisciplinary, multi-perspective analysis of issues and/or problems.

4. Does the course address the global learning course outcomes through active learning strategies?

The course must utilize active learning strategies (e.g. Team-Based Learning, case method of instruction, discussion, debate)

5. Does the course address the global learning course outcomes through an integrated co-curricular activity?

The course must include description of at least one co-curricular activity that addresses the global learning student learning outcomes. 
APPENDIX C

Draft Case Study, "The Case of Hoodia"

Spring 2009 
Multinational pharmaceutical firms commonly explore, extract, develop, and distribute drugs from traditional medicinal plants. Please read "The Case of Hoodia," a fictional account of a real-life dilemma that involves multiple stakeholders, including the San, the oldest continual human inhabitants of Africa, a multinational pharmaceutical firm, the health concerns of obese people around the world, a large pan-African government research organization, and a South African non-governmental organization.

After completing the reading, answer questions 1 and 2 .

"So, what do you do?"

\section{The Case of Hoodia}

Angela Bingham turned to her seatmate and tried to muster a genuine smile. Although she was proud of her work, Angela disliked being asked such a personal, invasive question by a stranger. Nevertheless, she was stuck sitting next to this man for the remainder of her 11-hour flight to Cape Town, so she decided to open up a little.

"I work for a company called Pharmedics. It's a British pharmaceuticals concern that specializes in developing drugs from traditional medicinal plants. The medicines we work on are used to treat asthma, Parkinson's disease, Alzheimer's disease, AIDS...you name it. My latest project is development of an extract from a plant called Hoodia Gordonii. It grows in the wild all over southern Africa and has been used by the San, or the Bushmen of the Kalahari, for thousands of years. The San are the first human inhabitants of Africa. They take Hoodia to stave off hunger and thirst on long hunting and gathering expeditions and during times of drought. The extract, P57, may turn out to be an anti-obesity wonder drug."

"Wow, that sounds interesting and like really good work. Are you a scientist?"

"No, I'm an account director. Actually, Pharmedics is a virtual company-there are very few of us who are employed directly by the company itself. I work with outsourced field researchers, lab scientists, clinicians, and manufacturers. I'm a middleman; I develop a communications strategy between the stakeholders and I coordinate feasibility studies for research and production. Pharmedics works on initial isolation of extracts. We leave the commercialization up to the big boys."

"The 'big boys'?"

“Yeah, Phizer, Unilever-big multinational pharmaceutical firms. They've got the money and the power to push drugs through the Food and Drug Administration and such. But tell me, what do you do, um...I can't believe I already forgot your name..."

Angela's seatmate smiled graciously. "Roger. Don't worry about it—l'm an artist, a sculptor, so I'm a little flighty myself. I'm bringing a commissioned work to Cape Town to be placed in front of the headquarters of a big shipbuilding company. I work with metal. The pieces of the sculpture are down in the baggage compartment. I'm going to South Africa to put them all together."

"Well, well," beamed Angela, "that's basically what l'm going to Cape Town to do, put together pieces. But I'm no artist. This is more like a sales job, although I'm not quite sure what l'm selling or to whom."

Temporarily saved from having to explain further by the arrival of the dinner cart, Angela leaned back in her seat and closed her eyes. She recalled the conversation she'd had the previous week with her company's president, David Campbell, when she was initially dispatched on this mission. 
"Angela, I just want you to know that you've done incredible work on the clinical trials of P57. It has enormous commercial potential and Phizer is very interested in taking it to the next level. But Angela, nothing can happen at all until we work things out with the San. I've got their lawyer, reporters from the Observer, a bunch of NGOs, and the governments of Namibia, Botswana, and South Africa breathing down my neck...it's unbelievable. I didn't even know the San existed anymore. I need you to go over there and make everybody happy."

Angela's heart pounded. She was used to bringing people together to work as a team, but this sounded much more complicated than what she usually did. "David, I'm not sure I understand what you want me to do. Why do we have a problem with the San? They don't have the development license on the patent for Hoodia, we do."

Taking off his glasses, David Campbell stood and began pacing the room. "We purchased the development rights for Hoodia from the initial patent holder, the South African-based Council for Scientific and Industrial Research (CSIR), one of the largest research organizations in Africa. Although it was a government-sponsored institution, it did not consult with the San, the original holders of the knowledge of Hoodia, before applying for the patent. Even if they had approached them, the San may have had little trust for an apartheid-era institution. They may not have even understood what was at stake for them. The San's way of life has been undermined by development in southern Africa. The San are poverty-stricken and they lack education and access to information, so they have little power to negotiate or profit from developing their indigenous knowledge... anyway, a South African NGO called BioWatch got wind of the CSIR agreement with us and leaked it to the press."

Angela was starting to catch on. "So do the San believe they are the true owners of Hoodia? Do they want some sort of monetary compensation for their knowledge of Hoodia?"

"To tell you the truth, the San find the very idea that anyone should pay them for their knowledge morally abhorrent. The San culture values knowledge as a collective resource. What's more, the whole patent process makes little sense to them. They don't see how life-even plant life_-can be 'owned."'

Sitting back down at his desk, Campbell went on to explain how matters were made even more complicated by the fact that the San were not a single community, but a group of multiple far-flung communities that lived and travelled throughout South Africa, Namibia, and Botswana. An advocacy organization had been formed in 1996 to lobby for the interests of the San communities, the Working Group of Indigenous Minorities in Southern Africa (WIMSA). Through their lawyer, WIMSA had recently informed Pharmedics and the CSIR of their decision not to pursue the San's 'no patents on life' policy in court, as it was too expensive. Instead, the San wanted to negotiate a benefits-sharing agreement, with Hoodia royalties being used to alleviate poverty and sustain endangered aspects of San culture. The distribution of such benefits was, however, potentially problematic. Even if an agreement could be reached between the CSIR, Pharmedics, and WIMSA, how could a system be created to fairly compensate multiple nomadic San groups across three countries?

Angela was overwhelmed but determined. "David, I can't believe what a puzzle you've placed in front of me. I'll go to Cape Town. I can't promise l'll make everyone happy, but l'll try to help everyone recognize all the many moving parts and how they can best fit together." 
"Ma'am, would you like eggs or French toast?"

Angela's attention snapped back into the present.

"Oh, uh, thank you. French toast, please." She looked away from the steward and over towards Roger. His dinner tray had been replaced with one featuring eggs and toast, and the sun was shining brightly through the window.

"Well good morning, sleepyhead, just in time for breakfast! You passed out without even taking a bite of dinner. I didn't want to wake you-I hope that's o.k. We've only got a few more hours before landing."

"Oh yes, of course. Roger, can I ask you something? You said you are going to Cape Town to put the pieces of your metal sculpture together. How exactly are you going to do that?"

"Well, you choose your method depending on the types of metals you are working with. If the metals are the same, you can weld them together. It takes a lot of heat and it's dangerous, but if you are careful the joining will last a long time. If the metals are different, it's very difficult to force them together with welding. You generally have to use some sort of fastener like bolts or rivets. You pick the process to match the parts."

"Thank you, Roger. I'm starting to think I should conceive of my task in Cape Town more in terms of sculpting than selling. You've helped me a lot."

Angela leaned back in her seat. She was grateful Roger had asked her what she did for a living; moreover, she was glad she'd chosen to open up to him. She smiled to herself, and this time it was genuine.

\section{QUESTION 1:}

Imagine yourself as a reporter for the International Herald Tribune writing a comprehensive article on Hoodia Gordonii. Who would you interview? What issues would you make sure to cover in your story?

\section{QUESTION 2:}

"The Case of Hoodia" concerns bioprospecting. Bioprospecting refers to the centuriesold practice of collecting and screening plant and other biological material for commercial purposes, such as the development of new drugs, seeds and cosmetics. Biopiracy is a negative term referring to the claiming of legal rights over indigenous knowledge, usually by means of patents, without compensation to the groups who originated the knowledge. Graham Dutfield has described fundamentally different views on biopiracy as follows:

In countries like India, the predominant view is that the nation itself is the "victim" of biopiracy. For Africa, the perception seems to be that the continent as a whole is prey to the biopiracies. But in the Americas, Australia and New Zealand, the victims are seen generally as indigenous peoples who usually-though not always-represent minority populations.

Comment on Dutfield's possible reasons for drawing these conclusions. 


\section{APPENDIX D}

Draft Case Study, "A Monumental Dilemma”

Spring 2009 
According to Dr. Martha Honey, co-founder and co-director of the Center on Ecotourism and Sustainable Development, ecotourism is travel to fragile, pristine, and usually protected areas that strives to be low impact and is often small-scale. It helps educate the traveler, provides funds for conservation, directly benefits the economic development and political empowerment of local communities, and fosters respect for different cultures and for human rights.

Please read "A Monumental Dilemma," a fictional account of a travel reporter's experience conducting research for a travel magazine article about ecotourism in Cambodia. After completing the reading, answer questions 1 and 2.

\section{A Monumental Dilemma}

It is 4:30 a.m. and as promised, my guide and driver, Kim San, is waiting for me at the hotel entrance. We had met the previous day to work out a sightseeing schedule for the week, and he insisted that the first thing I do on my tour of the Angkor Archaeological Park was witness the sunrise over Angkor Wat, the largest religious monument in the world.

I climb aboard Kim San's motorbike, and we're off. My heart races as we weave in and out of the streets of Siem Reap, the boomtown launching point for millions of yearly visitors to Angkor. In the darkness, the motorbike headlights reveal shadowy forms of men and women bustling to set up shops and restaurants that will serve the waking hordes of tourists.

It's a seven-kilometer drive to the main ticket booth to Angkor Wat. Kim San stops in front of a large, modern complex, built to move large crowds quickly through the concession.

Climbing off the bike, I look around. "Kim San, you said this place would be packed, but there's hardly anyone here."

Kim San smiles. "Many people wait to come until just before the sun rises. They are lazy. I have guided journalists before. I know you want to have the best view, and that is why I brought you here early. You will see. Believe me. Here, you must take a flashlight or you will trip and fall. You must purchase your ticket at the booth," says Kim San, "I will bring water. Follow me."

Looming in the distance, I sense the presence of Angkor Wat, though it lies nearly 2 kilometers away. Designated in 1993 as a United Nations Educational, Scientific, and Cultural Organization (UNESCO) World Heritage Site, Cambodia's Angkor Wat temple was also a finalist in the New Seven Wonders of the World competition in 2007. It is the best-preserved structure in the complex of over 1000 temples known collectively as Angkor, the Sanskrit word for city. Angkor flourished between the $9^{\text {th }}$ and $15^{\text {th }}$ centuries A.D. as the seat of the Khmer empire, which ruled over parts of present-day Laos, Thailand, Vietnam, Myanmar, and Malaysia. It was the largest preindustrial metropolis in the world, with a population of nearly one million and an urban footprint roughly the size of modern Los Angeles. Since its founding in the $12^{\text {th }}$ century, the temple complex of Angkor Wat has remained an active religious center, first dedicated to the Hindu god Vishnu, then re-dedicated to Theravada Buddhist use in the $14^{\text {th }}$ or $15^{\text {th }}$ century. It is a source of great national pride and has been depicted on every version of the Cambodian flag since 1863. 
Kim San leads me to a ticket window. At this hour, there are more employees and guards lingering about than tourists. I pay my \$60 US fee for a week's entrance to the park and am taken to a side room to have my photo taken for the pass. While waiting I do some quick mental calculations. In my background research, I read that there were nearly three million yearly visitors to Angkor. That's \$180 million US-a huge revenue source for a country with a Gross Domestic Product of only about \$10 billion US.

"Tickets are expensive, aren't they?" I comment to Kim San as we make our way back to his bike for the remaining 2-kilometer ride. "Angkor Wat brings in a huge amount of money to Cambodia."

"I guess so," he responds. "Cambodians get to enter for free, which is good, but no one really knows exactly where the money goes that is collected from foreigners. In 1999 the government gave a 10-year lease to a private company called Sokimex to handle all of the ticket sales in Angkor. A man named Sok Kong owns Sokimex, and he is a personal friend and creditor to Prime Minister Hun Sen and his family. Sokimex is supposed to give $\$ 10$ million US per year to Aspara, the government agency that oversees and manages the archaeological park. People think that most of that money actually ends up in the hands of corrupt government officials, because hardly any of it is spent to conserve the sites in the park."

"Is Angkor falling into disrepair?"

"Yes," agrees Kim San, "three million pairs of hands and feet brushing up against the sandstone bricks of the temples does a lot of damage, not to mention looting and vandalism, all of the waste produced, and the water used. Overuse of water destroyed the original city of Angkor, and now overuse is undermining the temples' sand foundation-the ground is literally sinking."

As we speed towards Angkor Wat, I realize I have a problem. The magazine dispatched me on this assignment to cover Angkor as an ecotourism site-to describe how tourism has helped revive Cambodia's ailing economy and preserve the local culture and environment. This information about ticket sales, temple destruction, and pollution seems to go against the ecotourism focus of my story.

Kim San stops along the long moat we'll have to cross to enter the main temple complex. As we walk, Kim San continues his commentary. "Most Cambodians are happy with the tourism, though, Joseph. Even the anchovy paste sellers in Siem Reap are making money. We are safe-the Khmer Rouge is gone-so most Cambodians feel that letting Sok Kong, Hun Sen, and their cronies keep the money is a small price to pay."

From my research, I know that Khmer Rouge is the name given to Cambodia's ruling party between 1975 and 1979. When the Khmer Rouge came to power in 1975, they declared that year the Year Zero. All Cambodian history and culture prior to Year Zero was to be destroyed and replaced by the new revolutionary culture, starting from scratch. Foreigners weren't allowed in the country; essentially, Cambodia was cut off from the rest of the world until 1992, when the United Nations began its peacekeeping mission.

When we reach the top of the tower, Kim San instructs me to find a place to sit comfortably. There's nothing to do now but wait for the sun to rise and reveal the view. In the stillness, I slowly become conscious of the sound of water buffalo moving through the waters of the moat and muffled chants of nearby monks. Over 100,000 people live within the boundaries of the archaeological park, making Angkor a living, breathing model of Cambodia's cultural heritage.

At last, the dawn breaks, the sun bathes the temple towers in a golden light, and thousands of intricate sculptures, carvings, and stone reliefs emerge from the shadows. 
I'm shocked out of my reverie by a group of tourists huffing and puffing up the steps behind us and fussing to their guide that they're late and they've missed the sunrise.

"I'm sorry, Madame. I'm afraid the sun waits for no one, not even someone who forgot her camera in the room."

I laugh to myself at the clever retort. l'd been impressed to learn through my background research that official guides like Kim San are certified by the National Tourism Agency of Cambodia. They all speak exceptional English, hold university degrees, and are steeped in the culture and history of the area. All this work earns them a daily rate of between $\$ 10$ and $\$ 20$ US per day-a king's ransom compared to the average Siem Reap salary of approximately $\$ 40$ US per month. Even off-duty policemen, paid approximately $\$ 30$ US per month, hang out around the temples, ready to guide those who decided against hiring someone in town.

The arrivals are increasing with the light. Busses are lining up on the other side of the moat and the souvenir sellers are beginning their steady sales pitch.

"You were right, Kim San, it's getting crowded around here. Shall we explore?"

A group of monks walk past, chanting and holding flowers, incense, and candles. The cameras click away. Kim San explains, "They are celebrating Magha Puja, a day of veneration for Buddha and his teachings. The ceremony is supposed to take place at night, but they perform it during the day, too, when the tourists are here. When they are done they will accept tips to have their pictures taken with the tourists. They use the money to fund a school; the monks teach local people the old crafts, and then people make things to sell here and in Siem Reap."

I turn and notice three little girls, bracelets and bamboo flutes in hand, standing in the middle of a group of shouting tourists.

"Canada! What's the capitol of Canada?"

"Ottawa!" responds one girl eagerly. "Ottawa in Ontario. Canada have 10 provinces. Ontario, Quebec, Nova Scotia, New Brunswick, Manitoba, British Columbia..." The child goes on to rattle off the rest of the provinces, plus their capitals and relative populations.

The crowd loves it. Video cameras whirr away, recording the scene.

"These kids are going to be on YouTube next week, aren't they?" I quip.

Kim San smiles. "They already are. They are earning money to pay their teachers, probably. The Khmer Rouge are gone, but we still have a big enemy in Cambodia: corruption. It is everywhere. Teachers charge children to enter the classroom, and even white-haired old women must pay off the army or police for the right to beg in the temples. We pay under the table for everything-birth certificates, travel visas, fair rulings from judges, everything. Everyone needs the money and everyone pays."

A little girl is tugging at my shirttail. "Handsome mister, where you from?"

"America," I respond.

"America, very good country. Capitol Washington, D.C. You buy flutes for your children? 2 flutes 2000 riels."

"I'll buy your flutes if you answer some questions for me," I bargain. "Tell me, do you go to school?"

"No. My brothers go to school. I earn money so they go to school."

"Why do your brothers go to school? What do they want to do when they grow up?"

"My brothers want to have a hotel. Make lots of money. They don't want to work on farm. Too hard work. No money. Now you buy flutes?" 
"Yes, now l'll buy your flutes." Digging deep in my pockets for the 2000 riels, I glance at Kim San, who, with his university degree, observes these interactions with detached amusement. I look back at the determined face of this little salesgirl, who, at 8 or 9-years-old, probably knows more geography than I do. I hand her the 2000 riels and turn around to look at Angkor Wat. With the sun rising behind it, it glows like a beacon of hope and casts a wide shadow below. At that moment, I know what the title of my article will be-Angkor Wat: A Monumental Dilemma.

\section{QUESTION 1:}

What is the "dilemma" that you think Joseph is writing about? What perspectives need to be taken into account in order to arrive at a solution to this dilemma?

\section{QUESTION 2:}

Donald O'Reilly, archeological advocate, has said, "We see tourism as the best way to preserve Cambodia's rich archaeological heritage." In contrast, John Stubbs of the World Monuments Fund has said, "Tourism is already out of control, and unless the Cambodian government takes some pretty radical action to reign it in now much of Angkor's magic and heritage could be lost forever." Given your knowledge of the forces currently affecting our world (historical, economic, political, social, environmental, etc.), do you think it is possible for Cambodia to preserve its cultural heritage through tourism? Please support your opinion with evidence of your knowledge of the forces affecting this issue. 
APPENDIX E

Draft Case Response Scoring Rubric

January 2009 


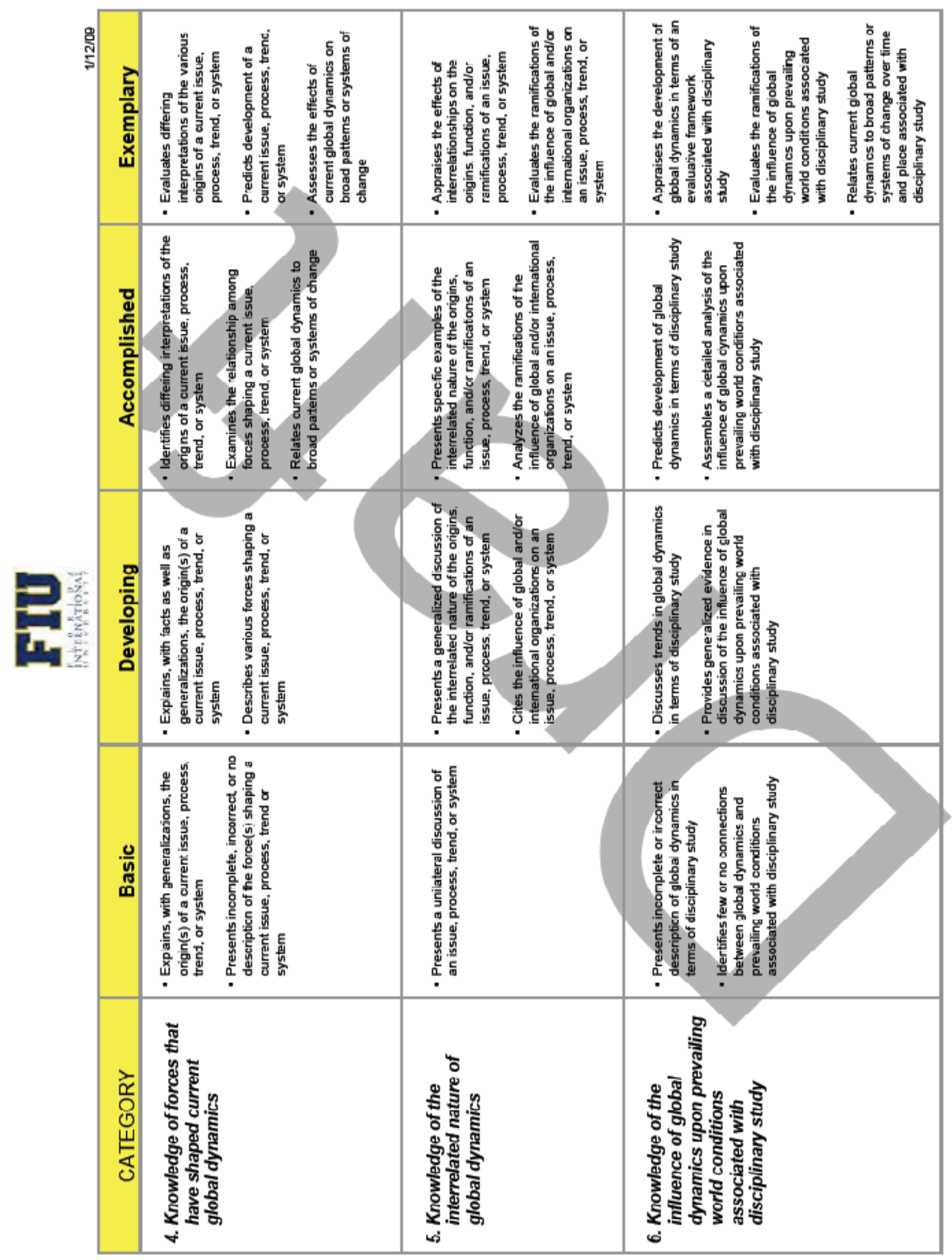




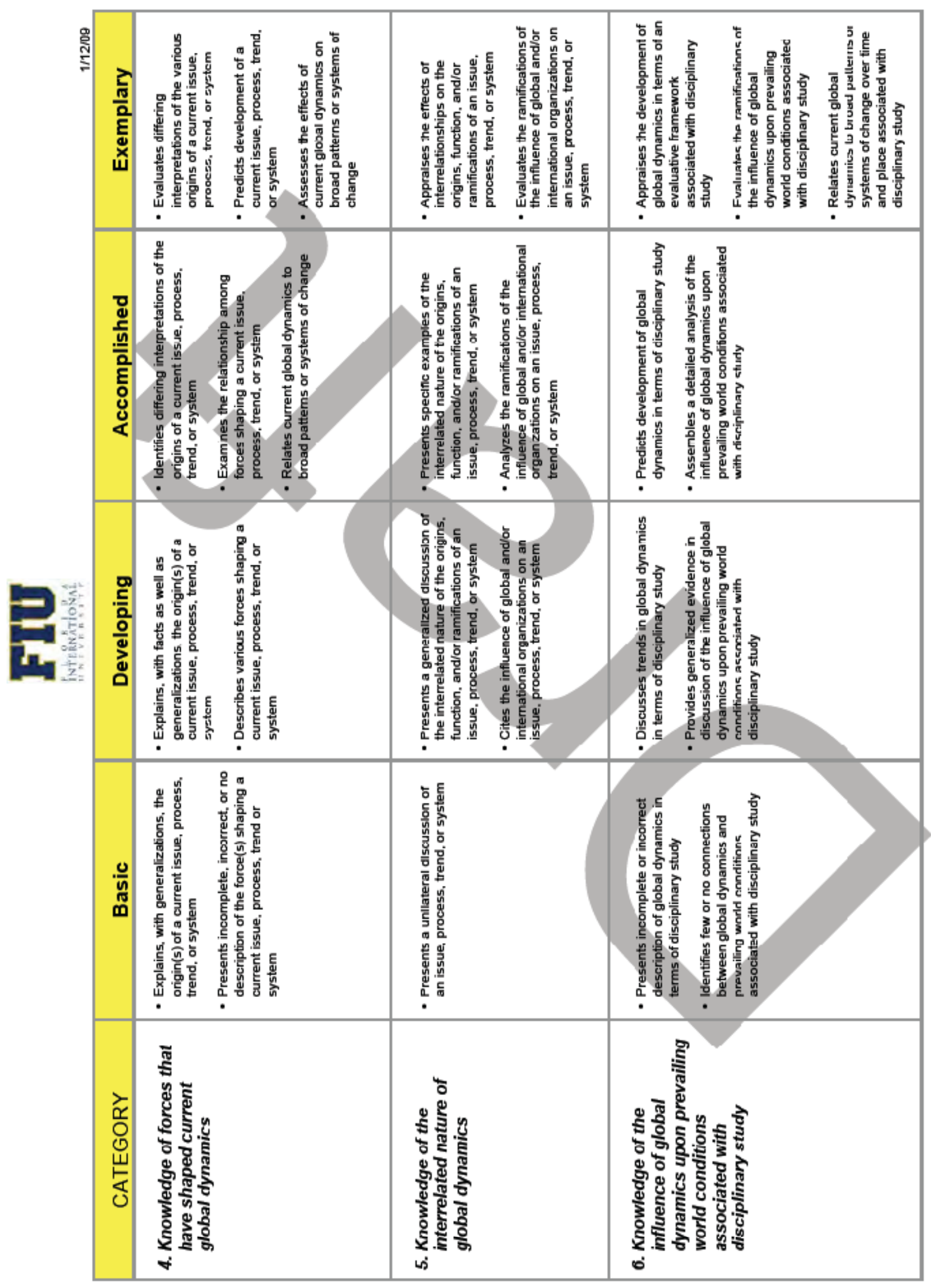




\section{APPENDIX F \\ Draft Case Response Scoring Rubric, "The Case of Hoodia"}

March 2009 


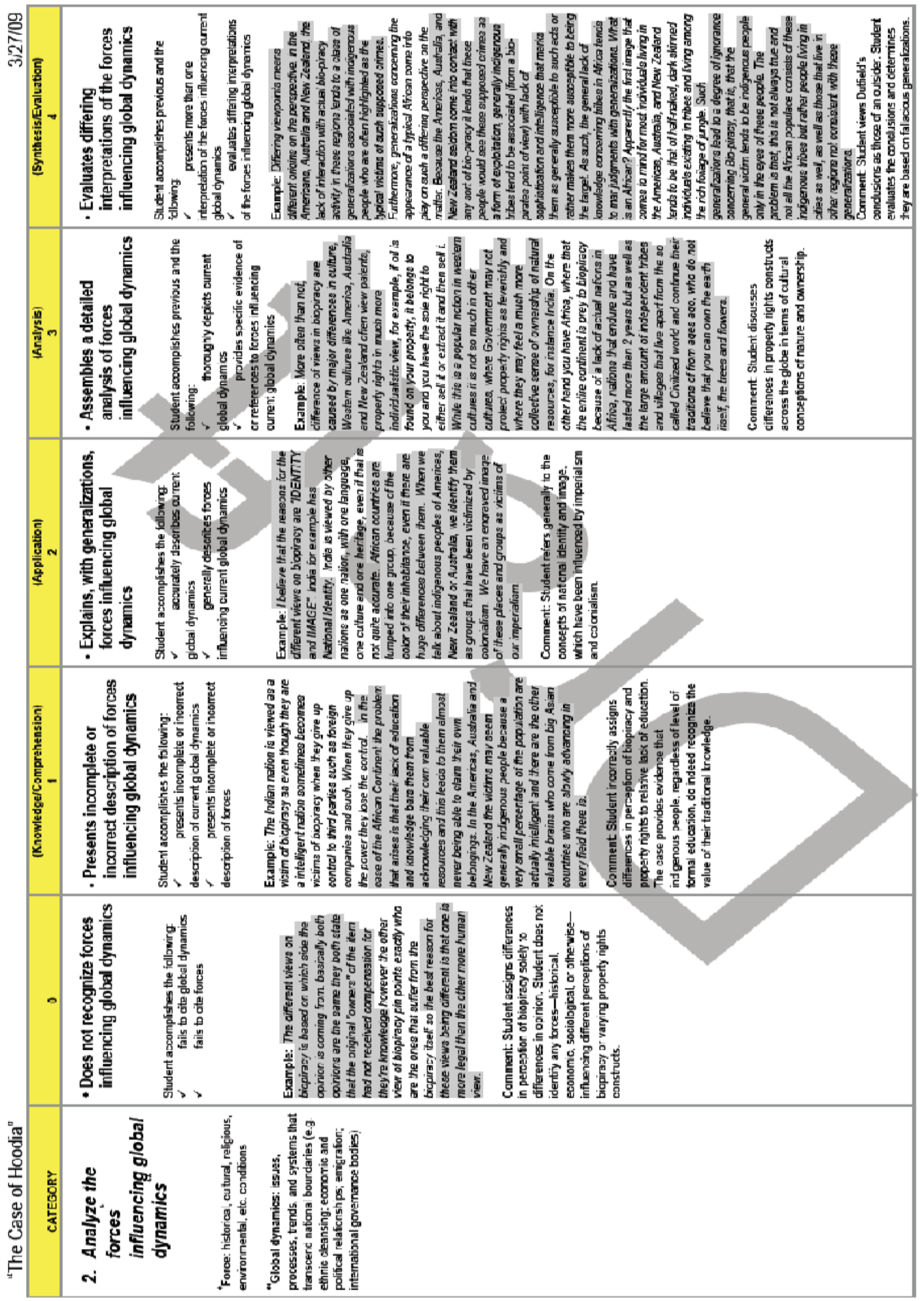




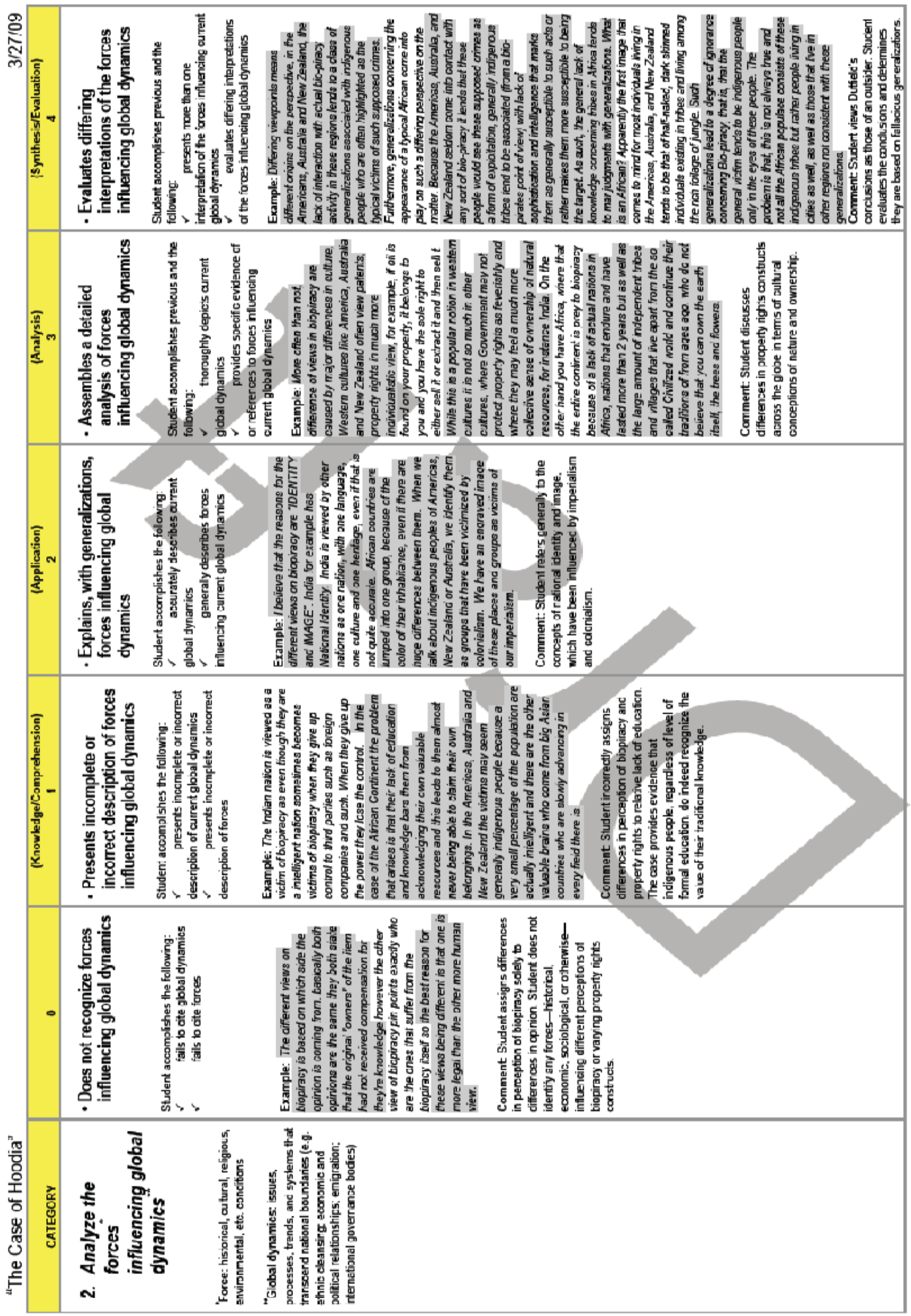




\section{APPENDIX G}

Draft Case Response Scoring Rubric, "A Monumental Dilemma"

April 2009 


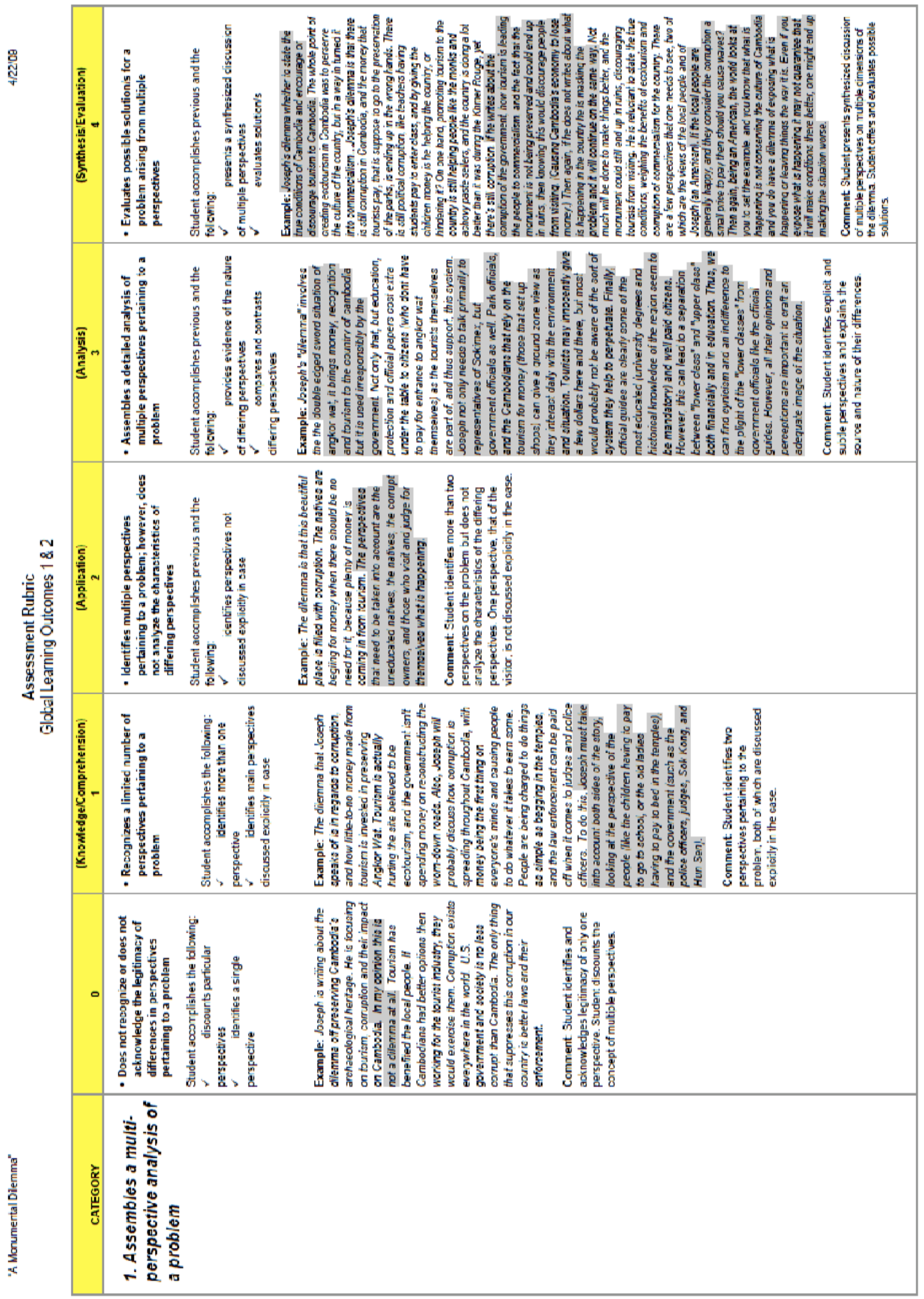




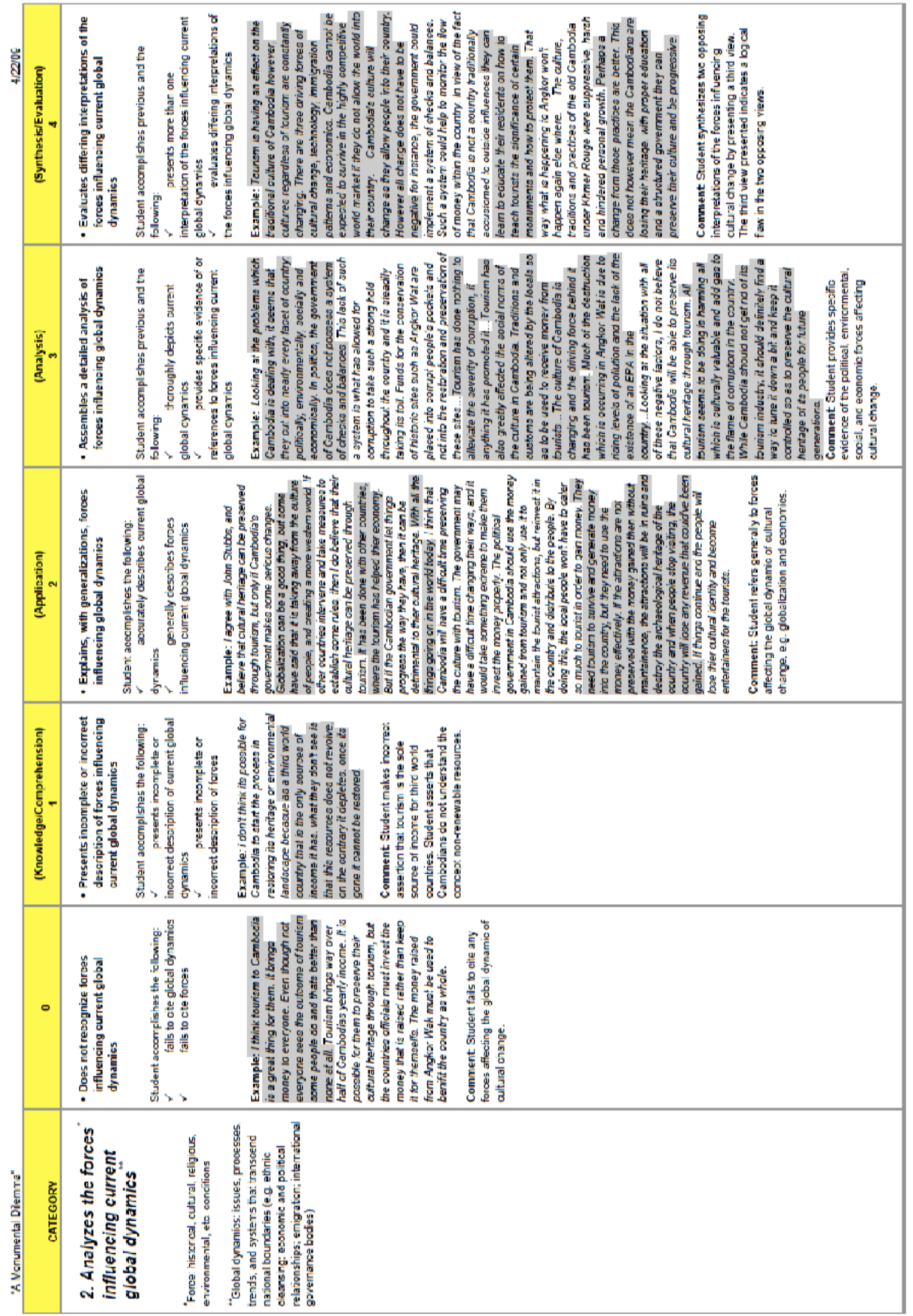


APPENDIX H

Case Response Assessment Pilot Faculty Survey

Spring 2009 


\title{
Case Response Assessment Pilot Faculty Survey, Spring 2009
}

\author{
Pilot Courses Taught \\ ENG 2012, Section A, Approaches to Literature \\ BOT 1010, Introductory Botany \\ WHO 2001, World Civilizations \\ ENG 2012, Section B, Approaches to Literature \\ COM 3461, Intercultural Communication
}

\author{
1. In your estimation, what percent of your total class time did you spend explicitly \\ addressing the following: \\ ENG 2012, Section A: Perspective Consciousness - 5, Knowledge of Global Dynamics - 5 \\ BOT 1010: Perspective Consciousness - 10, Knowledge of Global Dynamics - 10 \\ WHO 2001: Perspective Consciousness - 20, Knowledge of Global Dynamics - 70 \\ ENG 2012, Section B: Perspective Consciousness - 20, Knowledge of Global Dynamics - 10 \\ COM 3461: Perspective Consciousness - 40, Knowledge of Global Dynamics - 25
}

\section{Using your knowledge of both the "The Case of Hoodia" and perspective consciousness, do you believe there is enough content presented in the case to enable the student to answer this question?}

ENG 2012, Section A: Yes - Students responded to role playing aspect of the prompt. The facts of the case were less important to the response than the POV of the reporter herself. Internally, the case depicted the cognitive process it intended to measure.

BOT 1010: Yes

WHO 2001: Yes - Students can see many different perspectives from the reading -- San, corporations, government agencies, NGO aiding the San.

ENG 2012, Section B: Yes. The information is certainly available, but I even find myself struggling with the complexity and unfamiliarity of the "characters" involved. By this I mean that there are numerous entities involved in this story-- a group of people called the San, Pharmedics, Hoodia, WIMSA, BioWatch, CSIR, etc. One sentence on p. 5 says, "WIMSA had recently informed Pharmedics and the CSIR of their decision. . ." There's a communication or information absorption issue that arises here. At least according to the writing training I've received, people can only generally comprehend so many new "characters" (i.e. actors in a narrative) at once and there's a hierarchy of comprehensibility. People are the most comprehensible characters, new acronyms are a lot more difficult for readers to comprehend, assimilate into their own vocabulary, and then redeploy. So while the information is definitely available, the students' ability to comprehend and then use the different actors to answer this question is an concern. The students may have only been able to talk about the characters they had "met" before in other contexts-drug companies and maybe indigenous people like the San-- while they simply might not have been able to "upload" and "download" all the other actors into an answer for this question. COM 3461: Yes. The story discussed the issue of the San being nomadic--covering several different countries, Botswana, Namibia and South Africa. So not only do you have the perspective of the San, but each of the governments. The story discusses the issue of Biowatch, CSIR, WIMSA. A bit more detail about these organizations would have made it more obvious to the student that these agencies have a stake in the outcome. I would say this if this case was the first case at the start of the semester. If this case is used at the end of the semester, the students should be able to pick up on the interest of these agencies. Obviously, you have the pharmedics and the individuals in the story--Angela. 
3. Using your knowledge of perspective consciousness, do you believe this question measures the student's ability to assemble a multi-perspective analysis of a problem?

ENG 2012, Section A: Yes

BOT 1010: Yes

WHO 2001: Yes I think that this question is successful in eliciting student answers at different levels which accurately represent the understanding that students have of multiple perspectives. ENG 2012, Section B: Yes. Again, the information is there, but with all the different and unfamiliar actors, some of which are acronyms, in the story, I think it's difficult to attach the information about perspectives to these unfamiliar characters. In addition, because there are multiple actors in this very short story, it's a bit hard for the story to give thorough evidence of each actor's perspective. There probably needs to be some investigation into how we can present multiple perspectives in a story without overwhelming the student and ourselves with too many perspectives.

COM 3461: Yes. I feel the question is well written so as not to be too obvious. At the start of the semester, I would not expect that too many students would pick up on wanting to interview multiple stakeholders, but by the end of the semester they should be able to.

4. Using your knowledge of perspective consciousness, do you believe the rubric is a valid measure of the student's ability to assemble a multi-perspective analysis? In other words, do the levels of the rubric enable the scorer to distinguish between levels of achievement of this skill?

ENG 2012, Section A: Yes

BOT 1010: Yes

WHO 2001: Yes. Quite successfully. The variance in student answers shows this well.

ENG 2012, Section B: Yes.

COM 3461: Yes. Although catagory 4 (solutions) the question does not ask them to provide solutions.

5. Using your knowledge of both "The Case of Hoodia" and global dynamics, do you believe there is enough content presented in the case to enable the student to answer this question? ENG 2012, Section A: No - The tag line, 'Comment on Dutfield's reasons. . . ' is a distraction from the global dynamics variable itself. The prompt asks for an analysis of a perspective on another variable; thus 'global dynamics' is de-centered. Dutfield's response to the issues is thrust ahead of the student's evaluation of the issues.

BOT 1010: Yes

WHO 2001: No - The problem here is the quote from Dutfield. Students have to know a lot already about the different parts of the world in order to make sense of this.

ENG 2012, Section B: No. For better or for worse, this question will mostly demonstrate that the majority of our students have no understanding of the histories of the different continents and countries mentioned in the question. But I think this just ends up making our students (and us) look bad for the things they didn't learn in high school. I think we'll have to give a good deal of attention to delivering historical background in the cases themselves because we can't assume the kids have it.

COM 3461: No. I do not know how you could word it so as not to get off point, but I do not think the students understand what you were getting at. 
6. Using your knowledge of global dynamics, do you believe this question measures the student's ability to analyze the forces influencing current global dynamics?

ENG 2012, Section A: No

BOT 1010: Yes

WHO 2001: No. Some of the students need more information than they have in order to answer the question successfully.

ENG 2012, Section B: No. For same reasons as mentioned above.

COM 3461: No. Same as above.

7. Using your knowledge of global dynamics, do you believe the rubric is a valid measure of the student's ability to analyze the forces influencing global dynamics? In other words, do the levels of the rubric enable the scorer to distinguish between levels of achievement of this skill?

ENG 2012, Section A: Yes

BOT 1010: Yes

WHO 2001: No. Many of the student answers that were given low scores reflected a lack of knowledge about the different parts of the world. The Dutfield quote should be replaced.

ENG 2012, Section B: Yes

COM 3461: Yes

8. Using your knowledge of both "A Monumental Dilemma" and perspective consciousness, do you believe there is enough content presented in the case to enable the student to answer this question?

ENG 2012, Section A: Yes

BOT 1010: Yes

WHO 2001: Yes. The story is very good in presenting a wide variety of perspectives on tourism to sacred sites in Cambodia.

ENG 2012, Section B: Yes. There's certainly plenty of information here and it's easy to understand because it involves people, companies, governments, and places, but not acronyms. I'm still a little confused about why ecotourism is at issue here as a perspective rather than just plain old tourism, but I'll leave that to wiser heads than mine.

COM 3461: Yes. The story weaves in many perspectives at various points as the story unfolds.

9. Using your knowledge of perspective consciousness, do you believe this question measures the student's ability to assemble a multi-perspective analysis of a problem?

ENG 2012, Section A: Yes

BOT 1010: Yes

WHO 2001: Yes. The students had enough information about the perspectives of various social groups with regard to this tourism and its impact on the temple.

ENG 2012, Section B: Yes

COM 3461: Yes 
10. Using your knowledge of perspective consciousness, do you believe the rubric is a valid measure of the student's ability to assemble a multi-perspective analysis? In other words, do the levels of the rubric enable the scorer to distinguish between levels of achievement of this skill? ENG 2012, Section A: Yes

BOT 1010: Yes

WHO 2001: Yes. The rubric differentiated well between different levels of understanding perspective consciousness.

ENG 2012, Section B: Yes

COM 3461: Yes. The question for this story mentions the idea of having a solution to the dilemma.

11. Using your knowledge of both "A Monumental Dilemma" and global dynamics, do you believe there is enough content presented in the case to enable the student to answer this question?

ENG 2012, Section A: Yes

BOT 1010: Yes

WHO 2001: Yes. Students can see the problem from several sides. It does take imagination for students to figure out alternatives to the current practices.

ENG 2012, Section B: Yes. No problem here.

COM 3461: Yes. The fact that the question says (historical, economic, political, social, environmental) is a definate clue to my students waht you were getting at as these terms were part of my lectures.

12. Using your knowledge of global dynamics, do you believe this question measures the student's ability to analyze the forces influencing global dynamics?

ENG 2012, Section A: Yes

BOT 1010: Yes

WHO 2001: Yes. Students can clearly see the interactions of people from several countries in Cambodian tourism. They can see how the world is changing over time.

ENG 2012, Section B: Yes

COM 3461: Yes

13. Using your knowledge of global dynamics, do you believe the rubric is a valid measure of the student's ability to analyze the forces influencing global dynamics? In other words, do the levels of the rubric enable the scorer to distinguish between levels of achievement of this skill?

ENG 2012, Section A: Yes

BOT 1010: Yes

WHO 2001: Yes. The students have to bring a certain analytical and creative talent to this question, but this rubric accurately differentiates between different levels of understanding. ENG 2012, Section B:

COM 3461: Yes 


\section{APPENDIX I}

Outside Expert Judge Opinions of Case Response Assessment 


\section{Expert Judge \#1 \\ Dr. Kenneth Tye, College of Educational Studies, Chapman University}

-----Original Message-----

From: Tye, Kenneth [mailto:ktye@chapman.edu]

Sent: Wednesday, January 14, 2009 4:31 PM

To: Hilary Landorf

Subject: RE: global education case study assessment

Hillary,

I cannot open the case study, except in what looks like Vietnamese. I did view the rubrics and think they are superb. Resend me the case study. If it is as good as the rubrics, you should get a lot of mileage out of a separate publication, involving yourself and your team members.

There may be similar work in the field, but I am not aware of it. If there is, this adds to the field. If not, it is a breakthrough. I have had a number of inquiries of the years about assessment, but nothing with potential such as this.

I do recommend that you contact Ann Baker at the National Peace Corps Assn. to see if there is similar work. globaled@rpcv.org If there is, please let me know.

Ken

-----Original Message-----

From: Tye, Kenneth [mailto:ktye@chapman.edu]

Sent: Wednesday, January 14, 2009 5:17 PM

To: Hilary Landorf

Subject: RE: global education case study assessment

Hillary,

The case is "interesting." The degree to which it is an appropriate assessment tool in conjunction with the rubrics depends in great measure, upon what is taught and how it is taught. To put together a "package" the teaching issue needs to be addressed (I assume this represents assessment of some curriculum or "curriculum infusion.") That would be interesting to me. Also, I would be interested in knowing the results of a field test. Another question is age appropriateness. This is pretty sophisticated stuff. Is this directed at AP students, all 12th graders, or some other target population?

Finally, after field testing this package, do you have plans for developing a second case with additional questions, using the same rubrics? A whole lot of other questions would probably come to mind as other people read this material. I hope you are sending this out to others, also.

Ken 


\section{Expert Judge \#2 \\ Dr. Ethan Lowenstein, Department of Teacher Education, Eastern Michigan University}

-----Original Message-----

From: Ethan Lowenstein [mailto:elowenste@emich.edu]

Sent: Tuesday, January 13, 2009 1:11 PM

To: Hilary Landorf

Subject: Re: attachment for global learning

Hi Hilary,

I like the case study. In principle I like the rubric categories for goal\#1 and goal\#2.

However, I would be wary of using them, without first testing the validity of the instrument through a data-driven approach. This approach would involve asking 10-15

people who are not in the evaluation to respond to the case study, scoring the responses using your rubric and then developing "anchor responses" that can be attached to the rubric. For example, when someone scores in the acceptable category, what might a "typical response" look like. This will increase the reliability of scoring--i.e. scorers can have "anchors" to refer to while scoring responses. It will also increase validity by increasing the plausibility that the questions and case study are measuring what you are intending to measure and not something else. Another way to increase construct/external validity is to have a short narrative that accompanies the rubric that explains how the categories for scoring you've developed are grounded in the literature.

I hope that this feedback is helpful.

With warm regards,

Ethan

Ethan Lowenstein, Ph.D.

Associate Professor of Curriculum and Instruction

Department of Teacher Education

Eastern Michigan University

(734) 487-7120, ext. 2480 


\section{Expert Judge \#3 \\ Dr. William Gaudelli, Teachers College, Columbia University}

-----Original Message-----

From: Gaudelli, William [mailto:gaudelli@exchange.tc.columbia.edu]

Sent: Tuesday, January 20, 2009 10:14 PM

To: Andrew Gomez

Subject: RE: global learning assessment

Greetings Hilary,

Thanks for sharing this work with me. I have a few responses to what I generally find to be an interesting approach to assessment.

-The focus on global dynamics and systems seems appropriate, though not exclusive to the situation presented by the Hoodia case. Can there be a way to sharpen this so that it is solely focused on these dimensions? I certainly see human choice as part of this scenario as well but not addressed in the rubric.

-I'm not sure that the student is asked to use knowledge in the case of this scenario as I could imagine a student with little or no knowledge of pharmaceuticals responding appropriately to this question...isn't this as much a matter of ethics?

-I can't comment on the appropriateness of the domains themselves though I think this aspect of the assessment needs attention as it needs a fuller exploration of what 'perspective consciousness' looks/reads/sounds like in assessment terminology that is somewhat measurable.

Thanks for passing this on and good luck!

Bill

William Gaudelli

Associate Professor of Social Studies and Education

Project Leader for Teachers College to the Global Education Leadership Foundation

Teachers College, Columbia University

Zankel 420B

Box 80

525 W. 120th Street

New York, NY 10027-6696

(212) 678-3150

(212) 678-4118 FAX

gaudelli@tc.edu 
APPENDIX J

Final Draft Rubric, "The Problem with Hoodia" 


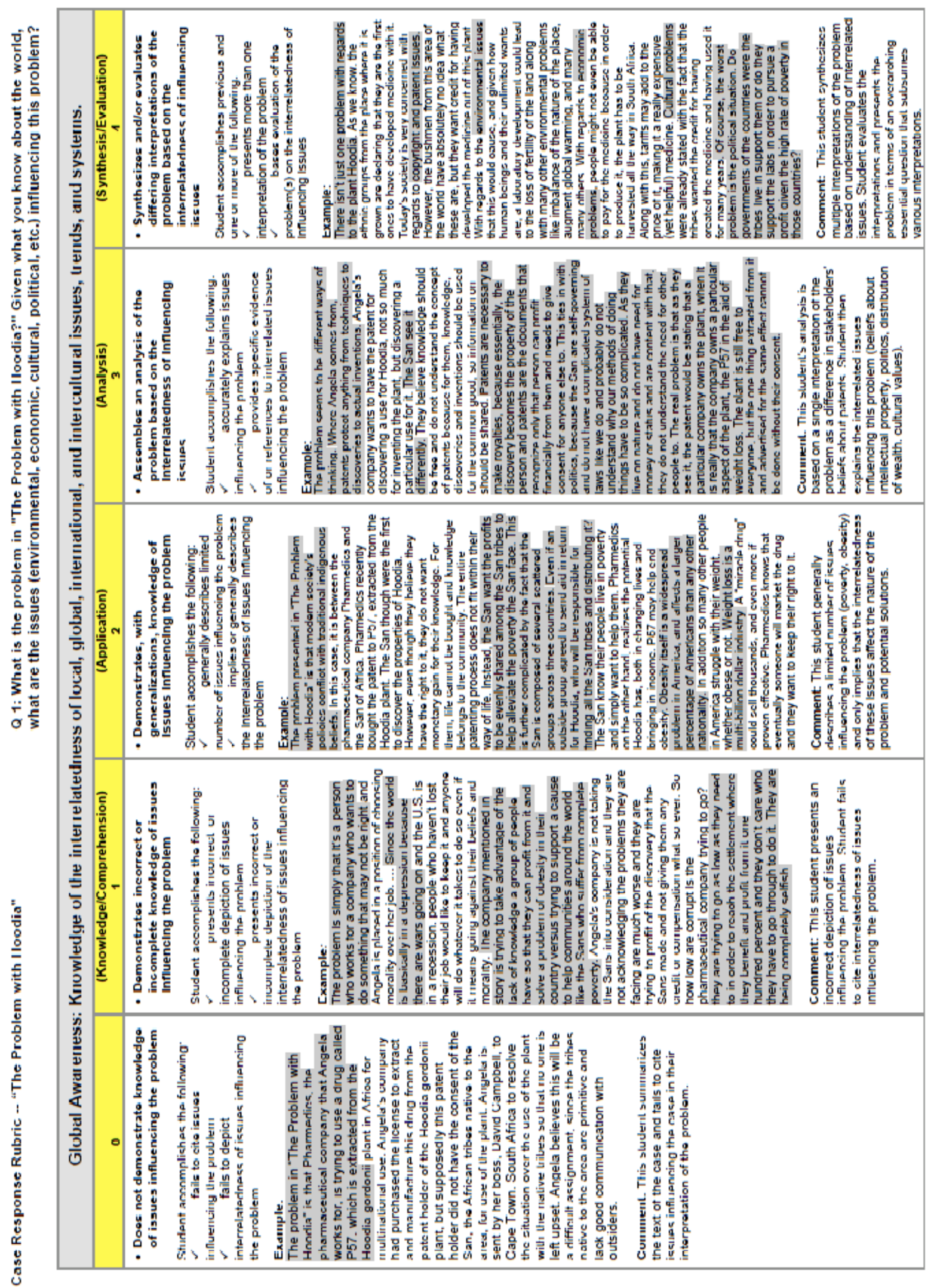




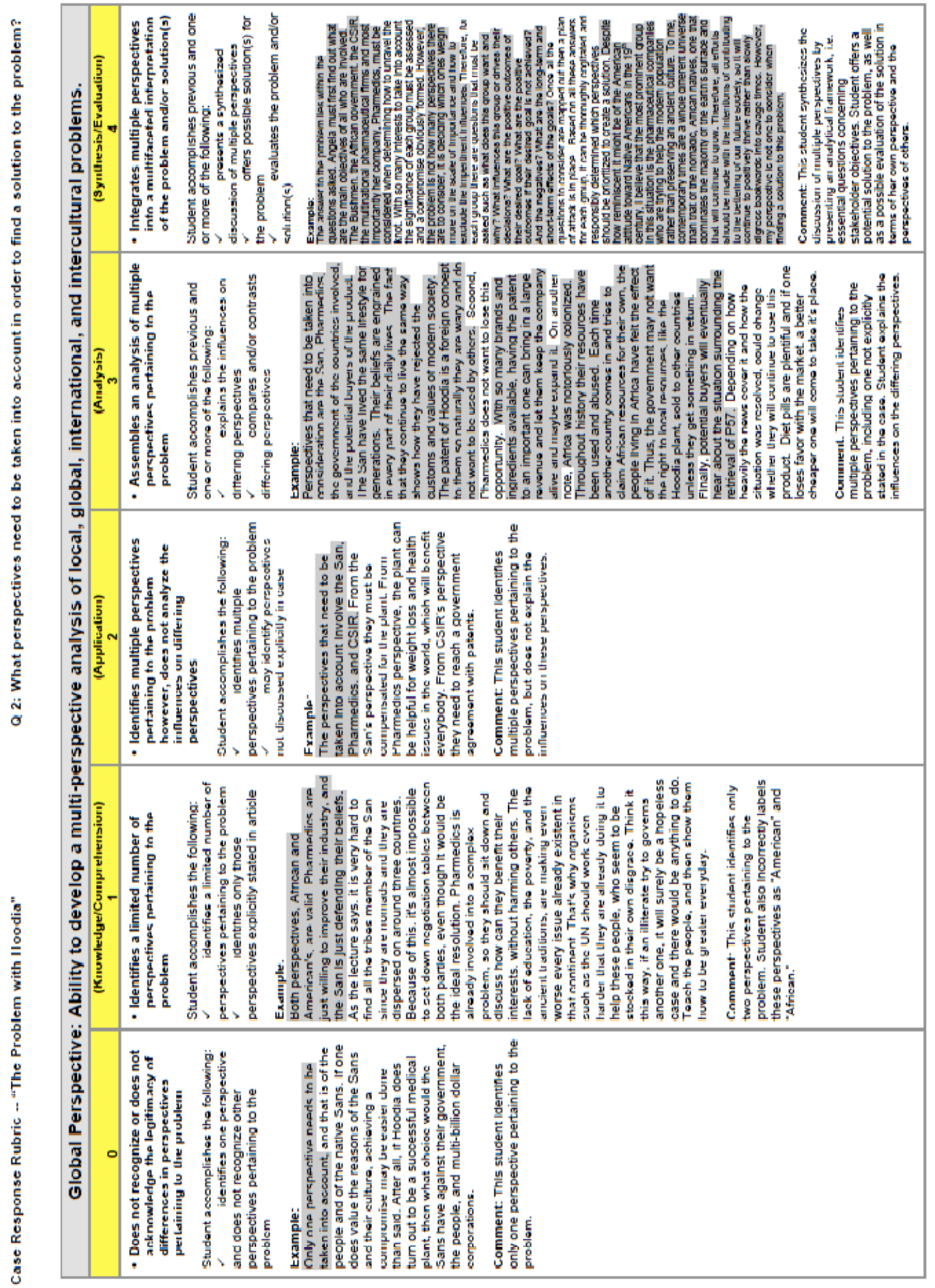




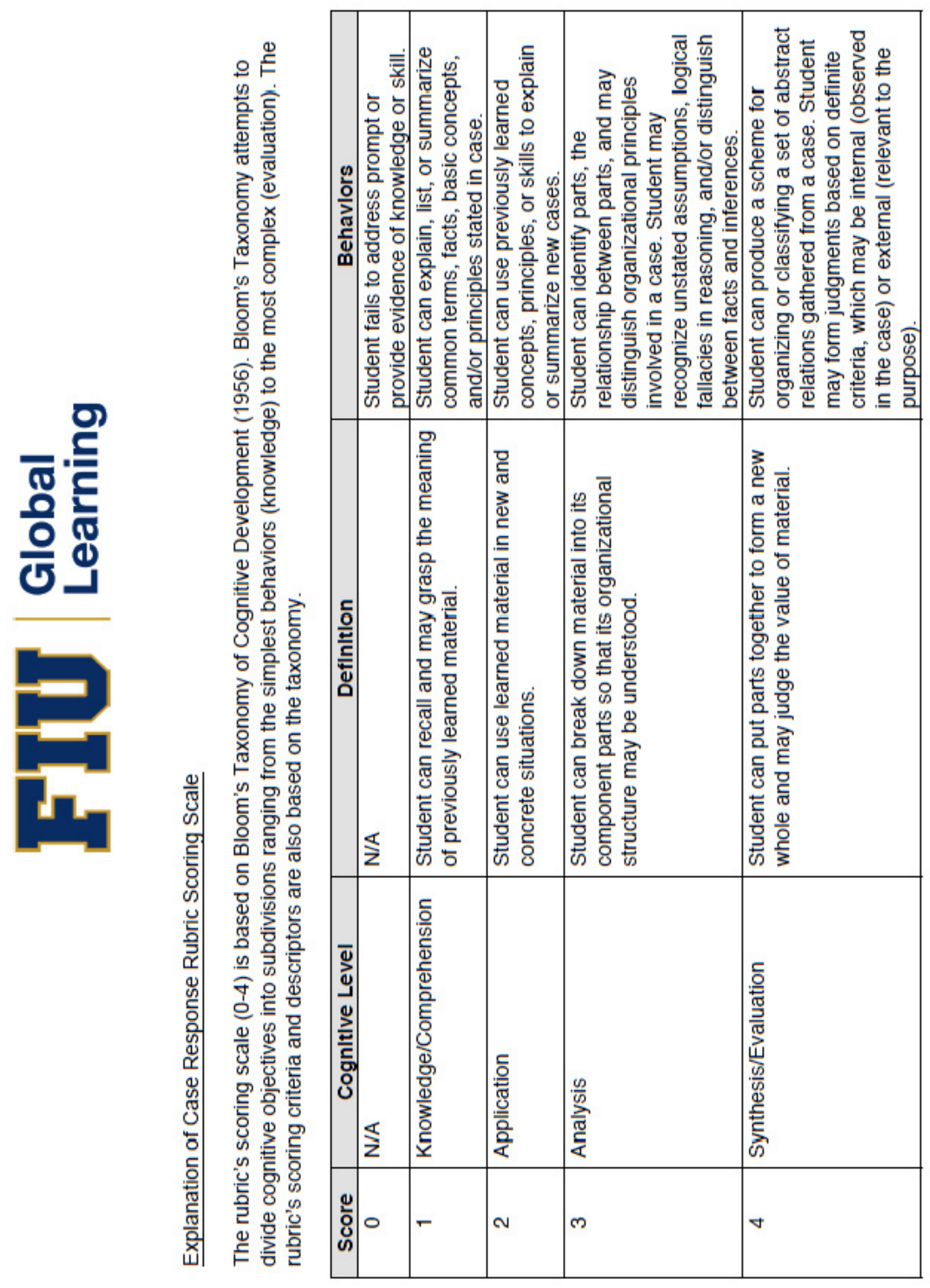


APPENDIX K

Final Draft Rubric, "A Monumental Dilemma" 


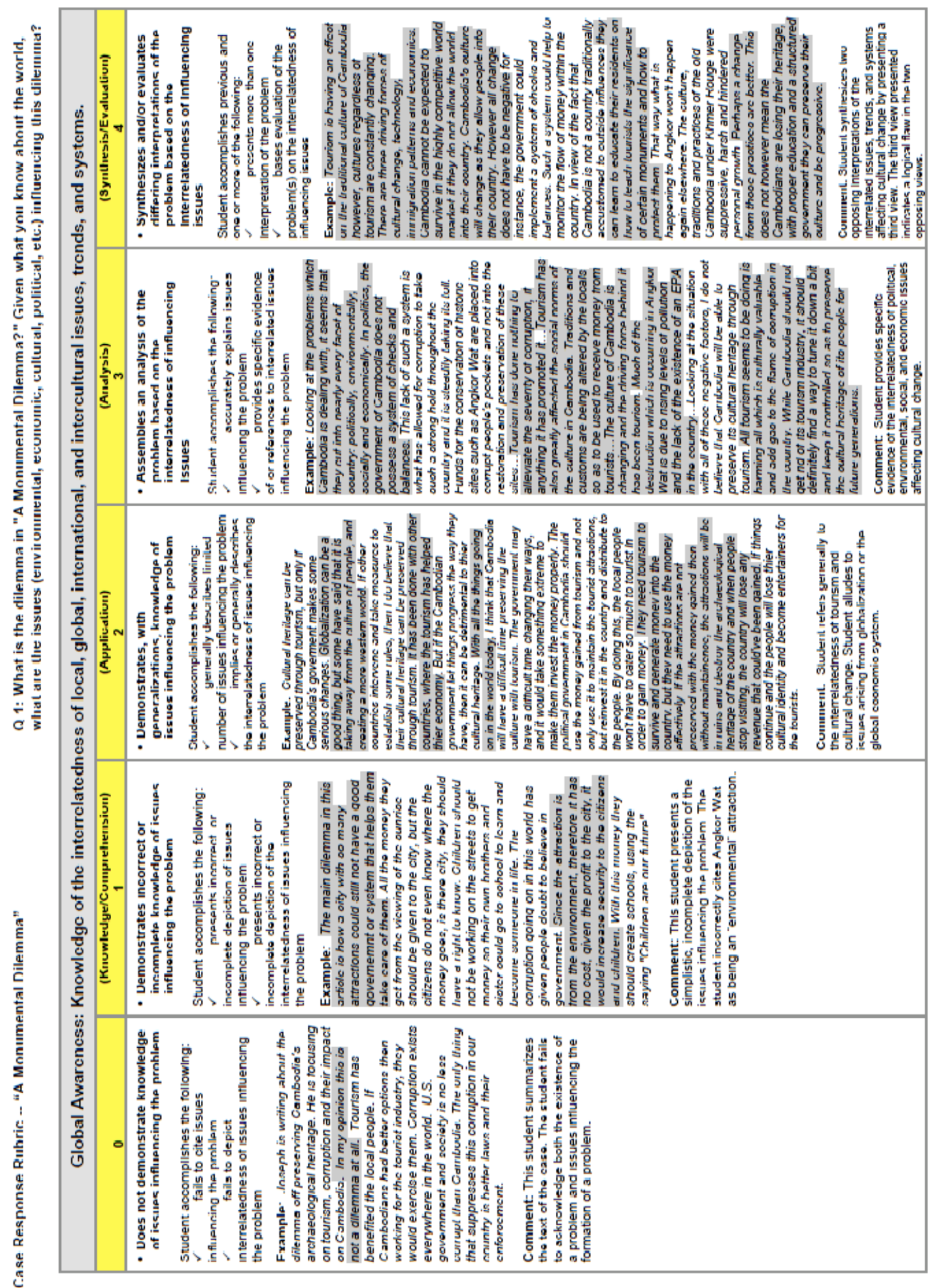




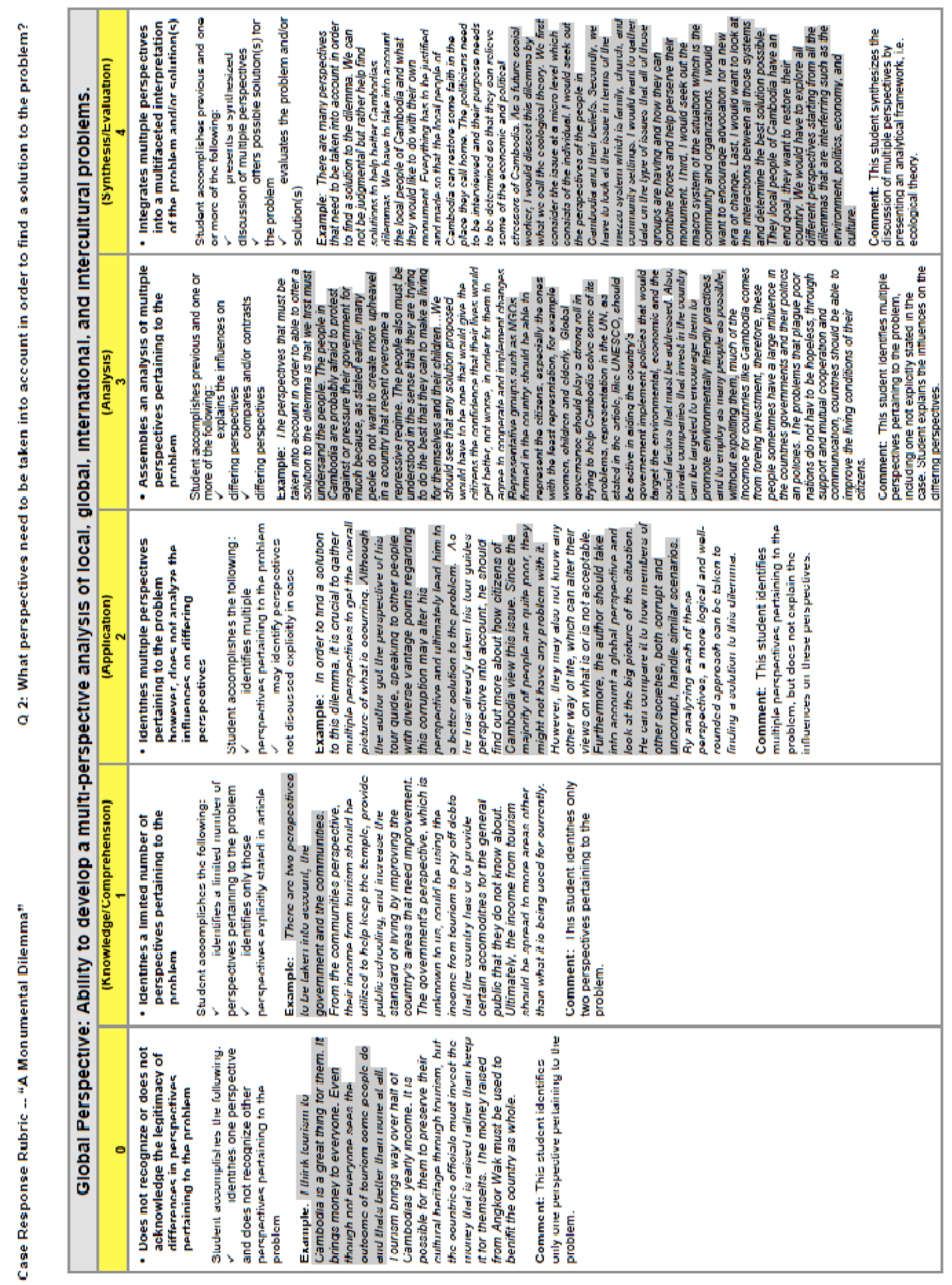




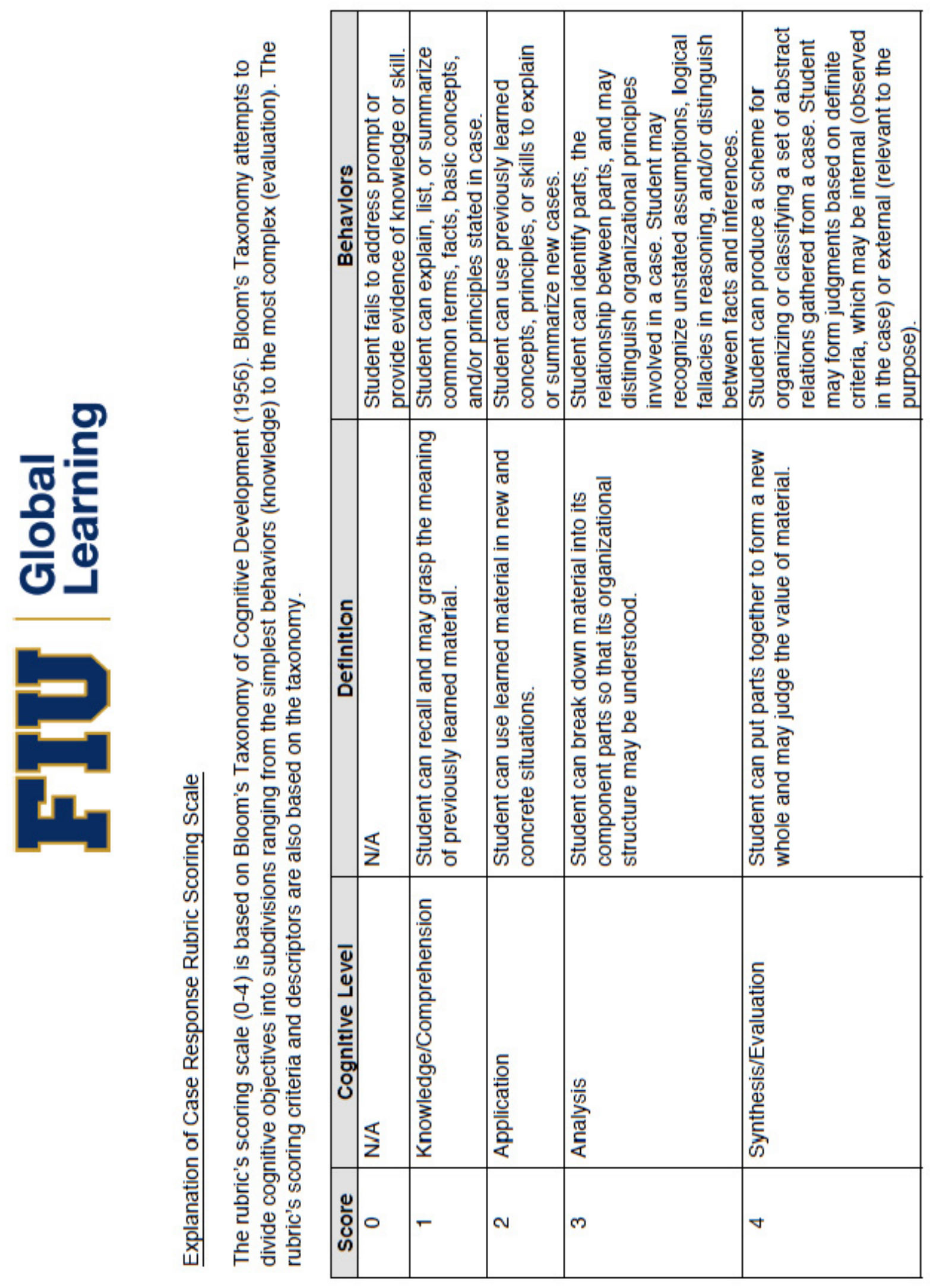


APPENDIX L

Sample Faculty Request Email 
From: Stephanie Doscher

Sent: Tuesday, December 28, 2010 3:52 PM

To: Peter J. Hargitai; James M. Sutton

Subject: Global Learning Assessment Study

Dear Professor Hargitai,

I am writing to you upon the recommendation of Dr. James Sutton.

FIU's QEP, Global Learning for Global Citizenship, has three student learning outcomes. These are:

Global Awareness: Students will be able to demonstrate knowledge of the interrelatedness of local, global, international, and intercultural issues, trends, and systems.

Global Perspective: Students will be able to conduct a multi-perspective analysis of local, global, international, and intercultural problems.

Global Engagement: Students will be able to demonstrate willingness to engage in local, global, international, and intercultural problem solving.

FIU is using two instruments as pre/post-assessments of the QEP's global learning SLOs. One of the instruments, the case response assessment, will be used to assess the first two SLOs and was developed in-house. In the spring, our office will be conducting a validity study of this assessment.

The purpose of the spring study is to determine the extent to which quantitative data supports the validity and reliability of assessment scores. Field tests have been very positive - this study will be a quasi-experimental comparison of scores from students enrolled in global learning and non-global learning courses.

I am writing to request your assistance in the delivery of the case response pre/postassessment in your three sections of Approaches to Literature. Since the assessment is a writing exercise, you may use the assessment artifact for students' Gordon Rule requirement. Our office will score the assessments according to the accompanying rubrics - the only investment participating you would need to make is the time necessary to deliver the assessment in class (two 45-minute sessions). We would like to deliver the pre-assessment within the first two weeks of class. The post assessment can be delivered within the final two weeks of class.

I have attached the pre/post instruments and the scoring rubric for your examination.

Thank you very much for your consideration. I'm happy to discuss this with you further, either over the phone or via email, at your convenience.

Very best,

\section{Stephanie Doscher}

Associate Director

11200 S.W. 8th Street, University Park, GL 470

Tel: $305-348-4146$

Fax: 305-348-1008 


\section{APPENDIX M}

Case Response Assessment Pretest 


\section{Case Response Pre-Assessment}

Multinational pharmaceutical firms commonly explore, extract, develop, and distribute drugs made from traditional medicinal plants used by indigenous people. Please read "The Problem with Hoodia," a fictional account of a real-life healthcare issue that involves multiple stakeholders.

After completing the reading, answer questions the demographic questions and questions 1 and 2 .

${ }^{* *}$ ANSWERS MUST BE AT MINIMUM 150 WORDS IN LENGTH. **

\section{"The Problem with Hoodia"}

"Hello, I'm Roger. So, what takes you to Africa? Work or pleasure?"

Angela Bingham turned to her seatmate and tried to muster a genuine smile. Although she was proud of her work, Angela thought it odd that a stranger would try to start a conversation by asking such a personal question. Nevertheless, she was stuck sitting next to this man for the remainder of the 11-hour flight to Cape Town, so she decided to open up and try to be friendly. A little small talk might even make the time pass more quickly...

"I'm Angela," she replied, shaking Roger's hand. "I'm going to Africa for work. My company, Pharmedics, is involved in pharmaceutical drug development. The medicines we work on are used to treat asthma, Parkinson's disease, Alzheimer's disease, AIDS...you name it."

"Sounds interesting. So, are you going to Africa to find a cure for a disease?" Roger asked.

"Well, sort of...my latest project involves an extract from a plant called Hoodia gordonii. It grows in the wild all over southern Africa and has been used by the San, or the Bushmen of the Kalahari, for thousands of years. The San are the first human inhabitants of Africa. They take Hoodia to diminish hunger and thirst on long hunting and gathering expeditions and during times of drought. Hoodia's extract, called P57, may turn out to be an anti-obesity wonder drug."

"That sounds like very good work. Obesity is a terrible health problem, an epidemic, especially in the States. Are you a scientist?"

"No, I'm a manager. Actually, Pharmedics is a virtual company-there are very few of us who are employed directly by the company itself. I work with outsourced field researchers, clinicians, and lab scientists all over the world. It's a British company, but I'm based in New York. I develop a communications strategy between the stakeholders and I coordinate feasibility studies for research and production. Pharmedics only works on initial isolation of extracts, though. We leave the actual drug development and commercialization up to the big boys." 
"The 'big boys'?"

"Yeah, Pfizer, Unilever-big multinational pharmaceutical firms. They've got the money and the power to create the drugs and push them through the American Food and Drug Administration and such. But tell me, what do you do, um...I can't believe I forgot your name already..."

Angela's seatmate smiled graciously. "Roger. Don't worry about it—l'm an artist, a sculptor. I'm bringing a commissioned work to Cape Town to be placed in front of the headquarters of a shipbuilding company. I work with metal. The pieces of the sculpture are all down in the baggage compartment. I'm going to South Africa to put them together."

"Well, well," beamed Angela, "that's basically what I'm going to Cape Town to do. I'm no artist, though. I'm supposed to figure out how a whole bunch of puzzle pieces fit together, even though I have no idea what the end product is supposed to look like."

Temporarily saved by the impending arrival of the dinner cart from having to explain further, Angela leaned back in her seat and decided to close her eyes for a moment. She recalled the conversation she'd had the previous week with her company's president, David Campbell, when she was initially dispatched on this mission.

"Angela, I want you to know that you've done incredible work coordinating the clinical trials of P57. It has enormous commercial potential for the development of weight loss drugs and Pfizer is very interested in taking it to the next level. But Angela, we've hit a major roadblock. We can't sell P57 to Pfizer until we work things out with the San. They are claiming that they have rights to the extract because they originally discovered its medicinal qualities. I've got their lawyer, reporters from the International Herald Tribune, a bunch of human rights organizations, and the governments of Namibia, Botswana, and South Africa breathing down my neck... it's an unbelievable mess. I didn't even know that Bushmen existed anymore. I need you to go over to South Africa, meet with the different groups, and make everybody happy."

Angela's heart pounded. She was used to bringing diverse people together from multiple countries to work as a drug development team, but this sounded much more complicated than what she usually did. "David, I'm not sure I understand what you want me to do. Why do we have a problem with the San? They don't have a license to P57, we do."

Taking off his glasses, David Campbell stood and began pacing the room. "We purchased the license to develop an extract from the initial patent holder for Hoodia gordonii plant, the Council for Scientific and Industrial Research (CSIR). Although it is a government institution sponsored by Namibia, Botswana, and South Africa, the CSIR did not consult with the San, who live in those countries, before applying for the patent.

Even if they had approached the San, they may not have cooperated because they don't trust the government. The San's nomadic way of life has been seriously endangered by 
development in southern Africa. The San are poverty-stricken and they lack education and access to information, so they have little power to negotiate or profit from developing their knowledge of medicinal plants such as Hoodia gordonii... anyway, a South African non-governmental organization called BioWatch found out about the CSIR agreement with Pharmedics and leaked it to the San and to the press. That's how this whole problem started."

Angela was starting to catch on. "So do the San believe they are the true owners of Hoodia? Do they want some sort of monetary compensation for their knowledge of Hoodia?"

"I wish it were that simple. To tell you the truth, the San find the very idea that anyone should pay them for their knowledge morally abhorrent. The San value knowledge as a collective resource. What's more, the whole patent process makes little sense to them. They don't see how life-even plant life-can be 'owned."”

Sitting back down at his desk, Campbell went on to explain how matters were made even more complicated by the fact that the San are not a single community, but a group of multiple far-flung nomadic communities that travel throughout South Africa, Namibia, and Botswana. Although the San decided not to pursue their 'no patents on life' beliefs in court, they did want to negotiate a benefits-sharing agreement, with Hoodia royalties being used to alleviate poverty and sustain endangered aspects of San culture. The distribution of such benefits was problematic. Even if an agreement could be reached between the CSIR, Pharmedics, and the San, how could a system be created to fairly compensate multiple nomadic San groups across three countries?

Angela was overwhelmed but determined. "David, I can't believe what a puzzle you've placed in front of me. There are so many groups involved...l'll go to Cape Town, but I can't promise I'll make everyone happy. I'll try to help everyone see how complicated this is and work out some sort of compromise." David sighed. "That's what we need, Angela, a compromise. Just remember, P57 could change a lot of lives for the better, but if we don't put the pieces together no one will benefit."

"Well good morning, sleepyhead, just in time for breakfast! You passed out without even taking a bite of dinner. I didn't want to wake you-I hope that's o.k. We've only got a few more hours before landing."

"Oh yes, of course. I didn't intend to fall asleep...Roger, can I ask you something? You said you are going to Cape Town to put the pieces of your metal sculpture together. How exactly are you going to do that?"

"Well, you choose your method depending on the types of metals you are working with. If the metals are the same, you can weld them together. It takes a lot of heat and it's dangerous, but if you are careful the joining will last a long time. If the metals are different, it's very difficult to force them together with welding. You generally have to use some sort of fastener like bolts or rivets. You pick the process to match the parts." 
Angela took a moment to consider this. "'Pick the process to match the parts.' Maybe I should think of my job in South Africa more as a sculpture than as a puzzle. Thanks, Roger. You've helped me a lot."

Angela leaned back in her seat. She was grateful Roger had asked her what she did for a living; moreover, she was glad she'd chosen to open up to him. She smiled to herself, and this time it was genuine. 


\section{Case Response Assessment}

\section{DEMOGRAPHIC QUESTIONS}

\section{Complete all items for your responses to count.}

1. Panther ID\#:

2. Gender (circle one): $\quad M \quad F$

3. My status at the college/university in which I am enrolled (circle one):

Freshman Sophomore Junior Senior

4. Select the ethnic identity that best describes you (circle one):
African-American
Hispanic/Latino
American Indian/Alaskan Native
Multiple ethnicities
Asian/Pacific Islander
Unknown/Other
European/White

5. How many languages do you speak fluently (circle one)?

One Two Three or more

6. What is the longest period of time you have spent abroad (circle one)?

None Two weeks or less More than two weeks

7. What is the primary reason you have travelled abroad (circle one)?

Academic Service Work Residence Tourism

8. Have you ever been a student in an International Baccalaureate (IB) or global education magnet program (circle one)?

Yes No

9. Have you ever taken a global learning course at FIU before (circle one)?

Yes No

10. Have you ever taken this assessment before (circle one)?

Yes No 
Panther ID \#

QUESTION 1:

What is the problem in "The Problem with Hoodia"? Given what you know about the world, what are the issues (environmental, economic, cultural, political, etc.) influencing this problem?

${ }^{* *}$ Answer must be at minimum 150 words in length.** 
Panther ID \#

QUESTION 2:

What perspectives need to be taken account in order to find a solution to the problem?

${ }^{* *}$ Answer must be at minimum 150 words in length. ** 


\section{APPENDIX N}

Case Response Assessment Posttest 


\section{Case Response Post-Assessment}

According to Dr. Martha Honey, Co-Director of the Center on Ecotourism \& Sustainable Development, ecotourism is "travel to fragile, pristine, and usually protected areas that strives to be low impact and (usually) small scale. It helps educate the traveler; provides funds for conservation; directly benefits the economic development and political empowerment of local communities; and fosters respect for different cultures and for human rights." Please read "A Monumental Dilemma," a fictional account of a real-life ecotourism issue that involves multiple stakeholders.

After completing the reading, answer questions 1 and 2.

${ }^{* *}$ ANSWERS MUST BE AT MINIMUM 150 WORDS IN LENGTH.**

\section{"A Monumental Dilemma"}

It is 4:30 a.m. and as promised, my guide and driver, Kim San, is waiting for me at the hotel entrance. We had met the previous day to work out a sightseeing schedule for the week. He insisted that I begin my tour of the 7Angkor Archaeological Park by watching the sun rise over Angkor Wat, the largest religious monument in the world.

I climb aboard Kim San's motorbike, and we're off. My heart races as we weave in and out of the streets of Siem Reap, the boomtown launching point for millions of yearly visitors to Angkor. In the darkness, the motorbike headlights reveal shadowy forms of men and women bustling to set up shops and restaurants that will serve the waking hordes of tourists.

It's a seven-kilometer drive to the main ticket booth to Angkor Wat. Kim San stops in front of a large, modern complex, built to move large crowds quickly through the concession.

Climbing off the bike, I look around. "Kim San, you said this place would be packed, but there's hardly anyone here."

Kim San smiles. "Many people wait to come until just before the sun rises. I have guided journalists before. I know you want to have the best view, and that is why I brought you here early. You will see, believe me. Here, you must take a flashlight or you will trip and fall. You must purchase your ticket at the booth," says Kim San. "I will bring water. Follow me."

Looming in the distance, I sense the presence of Angkor Wat, although it lies nearly 2 kilometers away. Designated in 1993 as a United Nations Educational, Scientific, and Cultural Organization (UNESCO) World Heritage Site, Cambodia's Angkor Wat temple was also a finalist in the New Seven Wonders of the World competition in 2007. It is the best-preserved structure in the complex of over 1000 temples known collectively as Angkor, the Sanskrit word for city. Angkor flourished between the 9th and 15th centuries A.D. as the seat of the Khmer empire, which ruled over parts of present-day Laos, Thailand, Vietnam, Myanmar, and Malaysia. It was the largest preindustrial metropolis in 
the world, with a population of nearly one million and an urban footprint roughly the size of modern Los Angeles. Since its founding in the 12th century, the temple complex of Angkor Wat has remained an active religious center, first dedicated to the Hindu god Vishnu, then re-dedicated to Theravada Buddhist use in the 14th or 15th century. It is a source of great national pride and has been depicted on every version of the Cambodian flag since 1863.

Kim San leads me to a ticket window. At this hour, there are more employees and guards lingering about than tourists. I pay my $\$ 60$ US fee for a week's entrance to the park and am led to a side room to have my photo taken for the pass. While waiting I do some quick mental calculations. In my background research, I read that there were nearly three million yearly visitors to Angkor. That's \$180 million US-a huge revenue source for a country with a Gross Domestic Product of only about \$10 billion US.

"Tickets are expensive, aren't they?" I comment to Kim San as we make our way back to his bike for the remaining 2-kilometer ride. "Angkor Wat brings in a huge amount of money to Cambodia."

"I guess so," he responds. "Cambodians get to enter for free, which is good, but no one really knows exactly where the money goes that is collected from foreigners. In 1999 the government gave a 10-year lease to a private company called Sokimex to handle all of the ticket sales in Angkor. A man named Sok Kong owns Sokimex, and he is a personal friend and creditor to Prime Minister Hun Sen and his family. Sokimex is supposed to give \$10 million US per year to Aspara, the government agency that oversees and manages the archaeological park. People think that most of that money actually ends up in the hands of corrupt government officials, because hardly any of it is spent to conserve the sites in the park."

"Is Angkor falling into disrepair?"

"Yes," says Kim San, "three million pairs of hands and feet brushing up against the sandstone bricks of the temples does a lot of damage, not to mention looting and vandalism, all of the waste produced, and the water used. Overuse of water destroyed the original city of Angkor, and now overuse is undermining the temples' sand foundation-the ground is literally sinking."

As we speed towards Angkor Wat, I realize I have a problem. The magazine dispatched me on this assignment to cover Angkor as an ecotourism site-to describe how tourism has helped revive Cambodia's ailing economy and preserve the local culture and environment. This information about ticket sales, temple destruction, and pollution seems to go against the ecotourism focus of my story.

Kim San stops along the long moat we'll have to cross to enter the main temple complex. As we walk, Kim San continues his commentary. "Most Cambodians are happy with the tourism. Even the anchovy paste sellers in Siem Reap are making money. We are safe-the Khmer Rouge is gone-so most Cambodians feel that letting Sok Kong, Hun Sen, and their cronies keep the money is a small price to pay for the improvement of our safety, economic standing, and cultural recognition throughout the world." 
From my research, I know that Khmer Rouge is the name given to Cambodia's ruling party between 1975 and 1979. When the Khmer Rouge came to power in 1975, they declared that year to be Year Zero. All Cambodian history and culture prior to Year Zero was to be destroyed and replaced by the new revolutionary culture, starting from scratch. Foreigners weren't allowed in the country; essentially, Cambodia was cut off from the rest of the world until 1992, when the United Nations began its peacekeeping mission.

When we reach the top of the tower, Kim San instructs me to find a place to sit. There's nothing to do now but wait for the sun to rise and reveal the view. In the stillness, I slowly become conscious of the sound of water buffalo moving through the waters of the moat and muffled chants of nearby monks. Over 100,000 people live within the boundaries of the archaeological park, making Angkor a living, breathing model of Cambodia's cultural heritage.

At last dawn breaks, the sun bathes the temple towers in a golden light, and thousands of intricate sculptures, carvings, and stone reliefs emerge from the shadows. I'm startled out of my reverie by a group of tourists huffing and puffing up the steps behind us and fussing to their guide that they're late and they've missed the sunrise.

"I'm sorry, Madame. I'm afraid the sun waits for no one, not even someone who forgot her camera in the room."

I laugh to myself at the clever retort. l'd been impressed to learn through my background research that official guides like Kim San are certified by the National Tourism Agency of Cambodia. They all speak exceptional English, hold university degrees, and are steeped in the culture and history of the area. All this work earns them a daily rate of between $\$ 10$ and $\$ 20$ US per day-a king's ransom compared to the average Siem Reap salary of approximately $\$ 40$ US per month. Even off-duty policemen, paid approximately $\$ 30$ US per month, hang out around the temples, ready to guide those who decide against hiring someone in town.

The arrivals are increasing with the light. Busses are lining up on the other side of the moat and the souvenir sellers are beginning their steady sales pitch.

"You were right, Kim San, it's getting crowded around here. Shall we explore?"

A group of monks walk past, chanting and holding flowers, incense, and candles. The cameras click away. Kim San explains, "They are celebrating Magha Puja, a day of veneration for Buddha and his teachings. The ceremony traditionally takes place at night. Nowadays the monks also perform the I ritual during the day to receive money from tourists. This money is used to fund a school where the monks teach traditional arts and crafts skills to the locals. The locals then make products to sell to the tourists. When the monks have finished performing their ceremony they will accept tips to have their pictures taken with the tourists."

I turn and notice three little girls, bracelets and bamboo flutes in hand, standing in the middle of a group of shouting tourists. 
"Canada! What's the capitol of Canada?"

"Ottawa!" responds one girl eagerly. "Ottawa in Ontario. Canada have 10 provinces. Ontario, Quebec, Nova Scotia, New Brunswick, Manitoba, British Columbia..." The child goes on to rattle off the rest of the provinces, plus their capitals and relative populations.

The crowd loves it. Video cameras whirr away, recording the scene.

“These kids are going to be on YouTube next week, aren't they?" I quip.

Kim San smiles. "They already are. They are most likely earning money to pay their teachers. The Khmer Rouge is gone, but we still have a big enemy in Cambodia: corruption. It is everywhere. Teachers charge children to enter the classroom, and even white-haired old women must pay off the army and police for the right to beg in the temples. We pay under the table for everything - birth certificates, travel visas, fair rulings from judges, everything. Everyone needs the money and everyone pays."

A little girl is tugging at my shirttail. "Handsome mister, where you from?"

"America," I respond.

"America, very good country. Capitol Washington, D.C. You buy flutes for your children? Two flutes 2000 riels."

"l'll buy your flutes if you answer some questions for me," I bargain. "Tell me, do you go to school?"

"No. My brothers go to school. I earn money so they go to school."

"Why do your brothers go to school? What do they want to do when they grow up?"

"My brothers want to have a hotel. Make lots of money. They don't want to work on farm. Too hard work. No money. Now you buy flutes?"

"Yes, now l'll buy your flutes." Digging deep in my pockets for the 2000 riels, I glance at Kim San, who, with his university degree, observes these interactions with detached amusement. I look back at the determined face of this little salesgirl, who, at 8 or 9years-old, probably knows more geography than I do. I hand her the 2000 riels and turn around to look at Angkor Wat. With the sun rising behind it, it glows like a beacon of hope and casts a wide shadow below. At that moment, I know what the title of my article will be-Angkor Wat: A Monumental Dilemma. 


\section{Case Response Assessment}

\section{DEMOGRAPHIC QUESTIONS}

\section{Complete all items for your responses to count.}

1. Panther ID\#:

2. Gender (circle one): $\quad M \quad F$

3. My status at the college/university in which I am enrolled (circle one):

Freshman Sophomore Junior Senior

4. Select the ethnic identity that best describes you (circle one):
African-American
Hispanic/Latino
American Indian/Alaskan Native
Multiple ethnicities
Asian/Pacific Islander
Unknown/Other
European/White

5. How many languages do you speak fluently (circle one)?

One Two Three or more

6. What is the longest period of time you have spent abroad (circle one)?

None Two weeks or less More than two weeks

7. What is the primary reason you have travelled abroad (circle one)?

Academic Service Work Residence Tourism

8. Have you ever been a student in an International Baccalaureate (IB) or global education magnet program (circle one)?

Yes No

9. Have you ever taken a global learning course at FIU before (circle one)?

Yes No

10. Have you ever taken this assessment before (circle one)?

Yes No 
Panther ID \#

QUESTION 1:

What is the problem in "A Monumental Dilemma"? Given what you know about the world, what are the issues (environmental, economic, cultural, political, etc.) influencing this problem?

${ }^{* *}$ Answer must be at minimum 150 words in length.** 
Panther ID \#

QUESTION 2:

What perspectives need to be taken account in order to find a solution to the problem?

${ }^{* *}$ Answer must be at minimum 150 words in length. ** 


\section{APPENDIX O}

Faculty Rater Instruction Letter 
Dear Faculty Rater,

Thank you for agreeing to serve as a rater of the case response assessment for FIU's Quality Enhancement Plan (QEP), Global Learning for Global Citizenship. Your skilled participation will advance the effectiveness of the QEP and is greatly appreciated.

You will be working with a team to score student responses in two four-hour sessions. The first 60 minutes of the first session will consist of training. The training will apprise you of your responsibilities as a scorer and will increase scoring agreement across team members.

To prepare for the training, please review the information below and the documents attached.

\section{QEP Case Response Assessment -- Description}

Activity

Students read a case narrative. After reading the narrative, students respond to two open-ended questions concerning the case. Suggested response length $=150$ words minimum per question.

Outcomes Assessed

The QEP has three student learning outcomes (SLOs). Two of these are assessed with the case response assessment*:

Global Awareness -- knowledge of the interrelatedness of local, global, international, and intercultural issues, trends, and systems (Question 1)

Global Perspective -- ability to develop a multi-perspective analysis of local, global, international, and intercultural problems (Question 2)

\section{Scoring}

Students will receive two final scores (0-4), one for each outcome/question. Scores are determined on the basis of two holistic rubrics (one for each question). The final score is an average of a minimum of two raters' scores (if the two raters' scores are discrepant by more than 1 , a third rater will read the response and the final score is an average of the three raters' scores).

*The third QEP SLO is Global Engagement -- willingness to engage in local, global, international, and intercultural problem solving. Global Engagement is assessed via another assessment, the Global Perspective Inventory (GPI) survey (https://gpi.central.edu/).

\section{Attachments}

1. Case narratives and questions prompts for "The Problem with Hoodia" (read by freshmen and transfers) and "A Monumental Dilemma" (read by graduating seniors). 2. Rubrics for both cases with explanation of scoring scale

If you have any questions prior to the first training and scoring session, please contact Hilary Landorf, Director, Office of Global Learning Initiatives, landorfh@fiu.edu, or 305348-241 
VITA

STEPHANIE PAUL DOSCHER

September 6, 1966

1984-1988

1991-1993

1995-2000

$2000-2006$

2006-2008

2008-2012
Born, Atlanta, Georgia

B.A., History

Emory University

Atlanta, Georgia

M.A., Secondary Education

Western Washington University

Bellingham, Washington

Teacher

Fern Ridge Middle School

Elmira, Oregon

Media Specialist

Gulliver Academy

Miami, Florida

Teacher

Devon Aire K-8 Center

Miami, Florida

Associate Director, Office of Global Learning Initiatives Florida International University

Miami, Florida

\section{PUBLICATIONS AND PRESENTATIONS}

Doscher, S. P., \& Wells, L. M. (2012, March). Coherence and continuous improvement: The development and implementation of Global Learning for Global Citizenship. Presentation at the American Association of Colleges and Universities' Network for Academic Renewal Conference, Seattle, WA.

Doscher, S. P. (2008). Knowledge vs. national security: The case of Androscoggin High School. Journal of Cases in Educational Leadership, 11(1), 97-105.

Doscher, S. P. (2006). Ethical leadership and decision making in education: Applying theoretical perspectives to complex dilemmas. Journal of Educational Administration, 44(5), 522-525.

Doscher, S. P., \& Normore, A. H. (2008). The moral agency of the educational leader in times of national crisis and conflict. Journal of School Leadership, 18(1), 8-31. 
Landorf, H., \& Doscher, S. P. (in press). Global Learning for Global Citizenship. In M. Walker and A. Boni (Eds.) Universities and Human Development. A Sustainable Imaginary for the XXI Century. New York, NY: Routledge Press.

Landorf, H. \& Doscher, S. P. (2012, April). Cultural context of comparison and global citizenship. Presentation at the $56^{\text {th }}$ Annual Conference of the Comparative and International Education Society, San Juan, PR.

Landorf, H. \& Doscher, S. P. (2012, April). Can an institution of higher education gain the necessary capabilities to educate students for global citizenship? Presentation at the $56^{\text {th }}$ Annual Conference of the Comparative and International Education Society, San Juan, PR.

Landorf, H., \& Doscher, S. P. (2012, Spring). Promoting engagement in curriculum internationalization. IIE Networker, 36.

Landorf, H., \& Doscher, S. P. (2010, February). Comprehensive planning for curriculum internationalization. Presentation at the annual meeting of the American Council on Education Internationalization Collaborative, Arlington, VA.

Landorf, H., \& Doscher, S. P. (2009, May). Campus internationalization? Yes, we can! Poster presented at the annual NAFSA: Association of International Educator Conference and Expo, Los Angeles, CA.

Landorf, H., \& Doscher, S. P. (2007, February). Human capability: The twenty-first century approach to education. Presentation at the $51^{\text {st }}$ Annual Comparative and International Education Society Conference, Baltimore, MD.

Landorf, H., Doscher, S. P., Duke, S., \& Gottlieb, E. (2011, February). Breaking boundaries: Collaborating across the university(s) to educate global citizens. Presentation at the annual conference of the Association of International Education Administrators, San Francisco, CA.

Landorf, H., Doscher, S. P., \& Rocco, T. (2008). Education for sustainable human development: Towards a definition. Theory and Research in Education, 6(2), 221236.

Landorf, H., Scorza, J., Omolo, M., \& Doscher, S. P. (2012, February). Engaging global citizenship through institutional culture and general education. Presentation at the annual conference of the Association of International Education Administrators, Washington, DC.

Normore, A. H., \& Doscher, S. P. (2007). Using media as the basis for a social issues approach to promoting moral literacy in university teaching. Journal of Educational Administration, 45(4), 427-450. 